Supporting Information

\title{
Functionalization at Nonperipheral Positions of Triazatruxene: Modular Construction of 1,6,11-Triarylated- Triazatruxenes for Potentially Organic Electronics and Optoelectronics
}

\author{
Murat Aslan, Yunus Taskesenligil, Selin Pıravadıl1, Nurullah Saracoglu* \\ *Department of Chemistry, Faculty of Sciences, Atatürk University, Erzurum, 25240, Turkey \\ E-mail: nsarac@atauni.edu.tr
}

\section{Table of contents}

1. ${ }^{1} \mathrm{H}$ NMR $(400 \mathrm{MHz})$ and ${ }^{13} \mathrm{C}\{1 \mathrm{H}\} \mathrm{NMR}(100 \mathrm{MHz})$ spectra of compounds $\mathrm{S} 2-\mathrm{S} 42$

2. Cartesian coordinates and total energy of optimized structure S43-S49

3. HRMS spectra of compounds S49-S62 


\section{1. ${ }^{1} \mathrm{H}$ NMR (400 MHz) and ${ }^{13} \mathrm{C}\{1 \mathrm{H}\}$ NMR (100 MHz) spectra of compounds}

\section{7-Bromoindolin-2-one (4)}

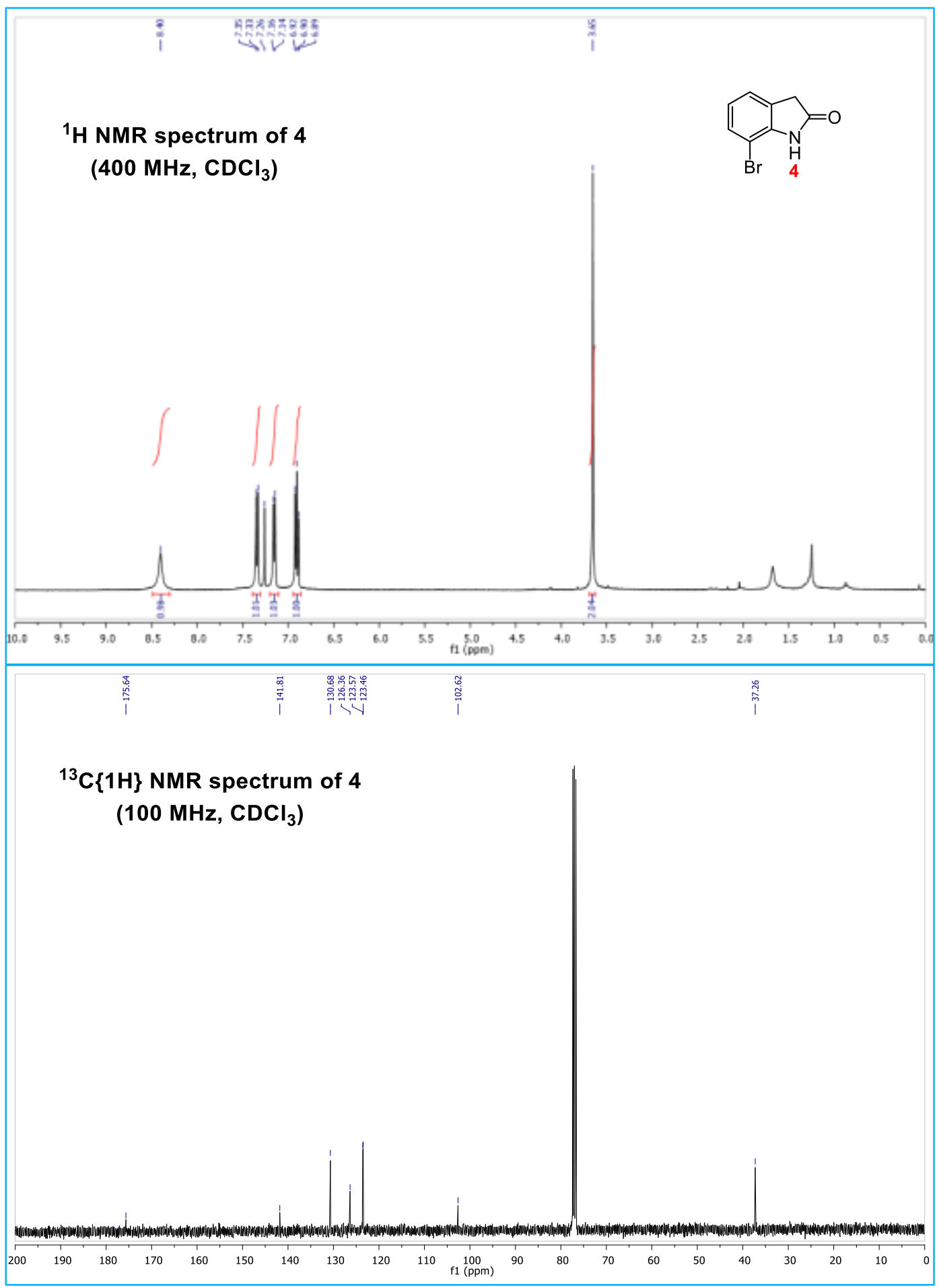


7-Phenylindolin-2-one (5)

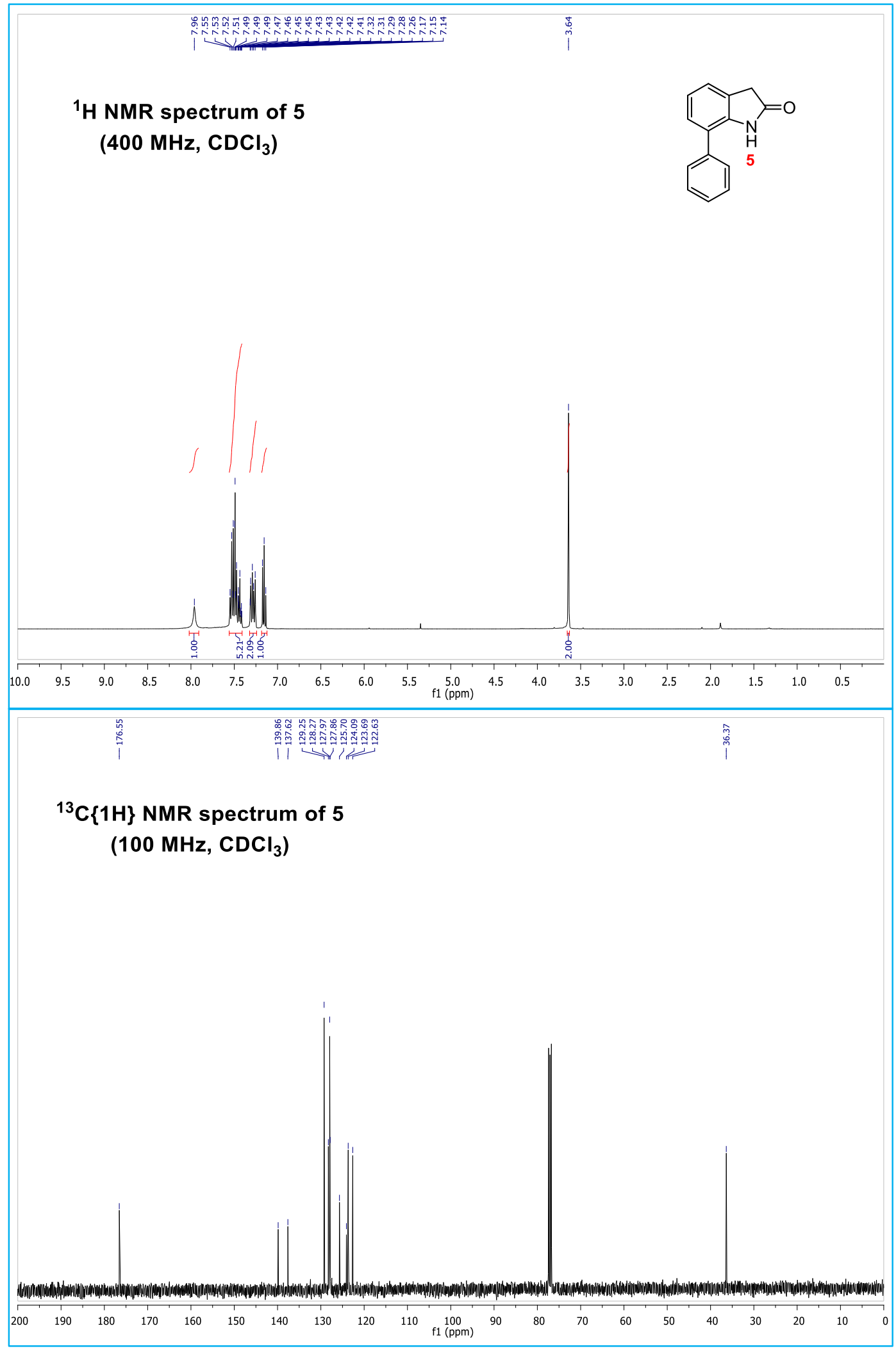


7-Bromo-1-ethylindoline-2,3-dione (6)

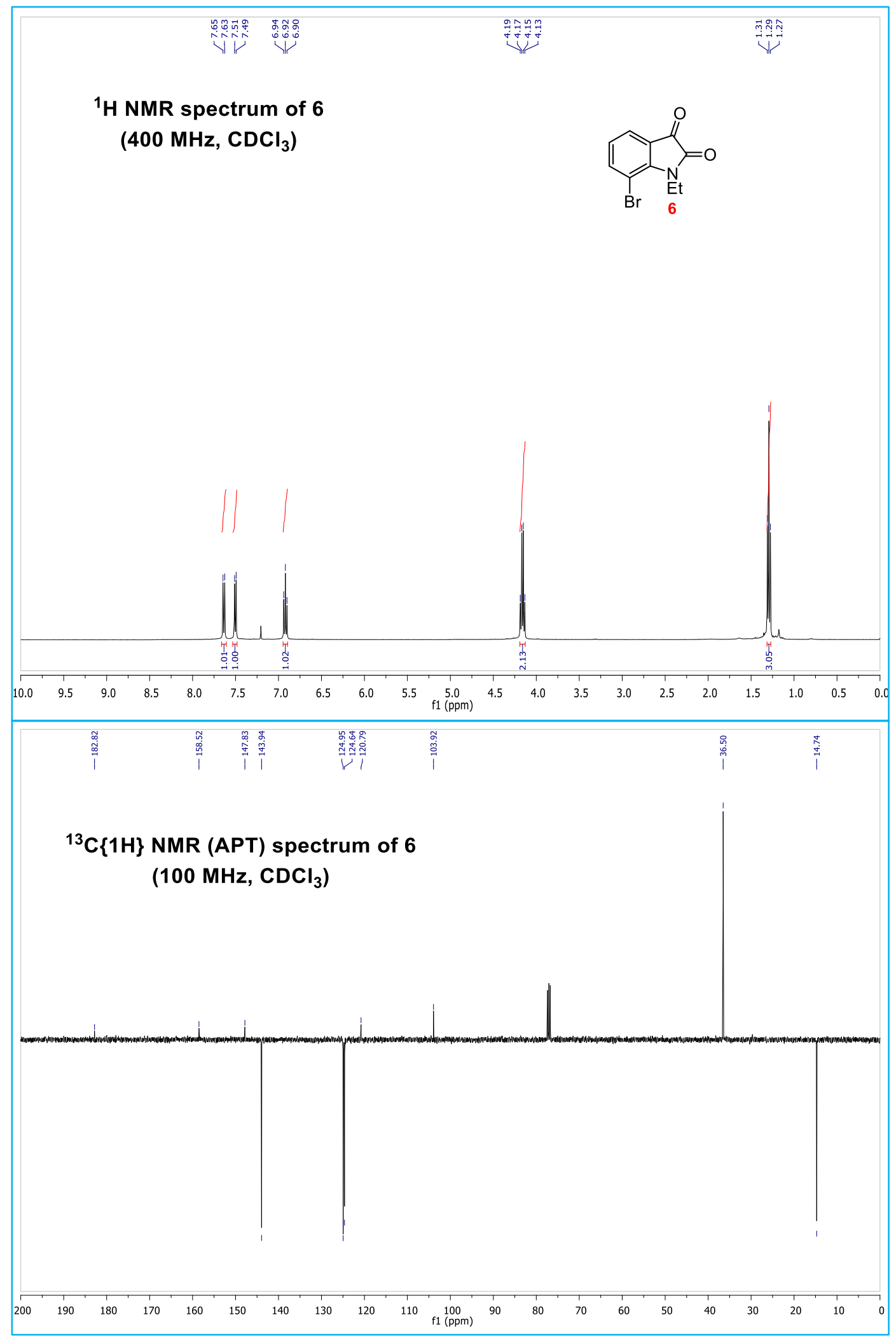


7-Bromo-1-ethylindolin-2-one (7)

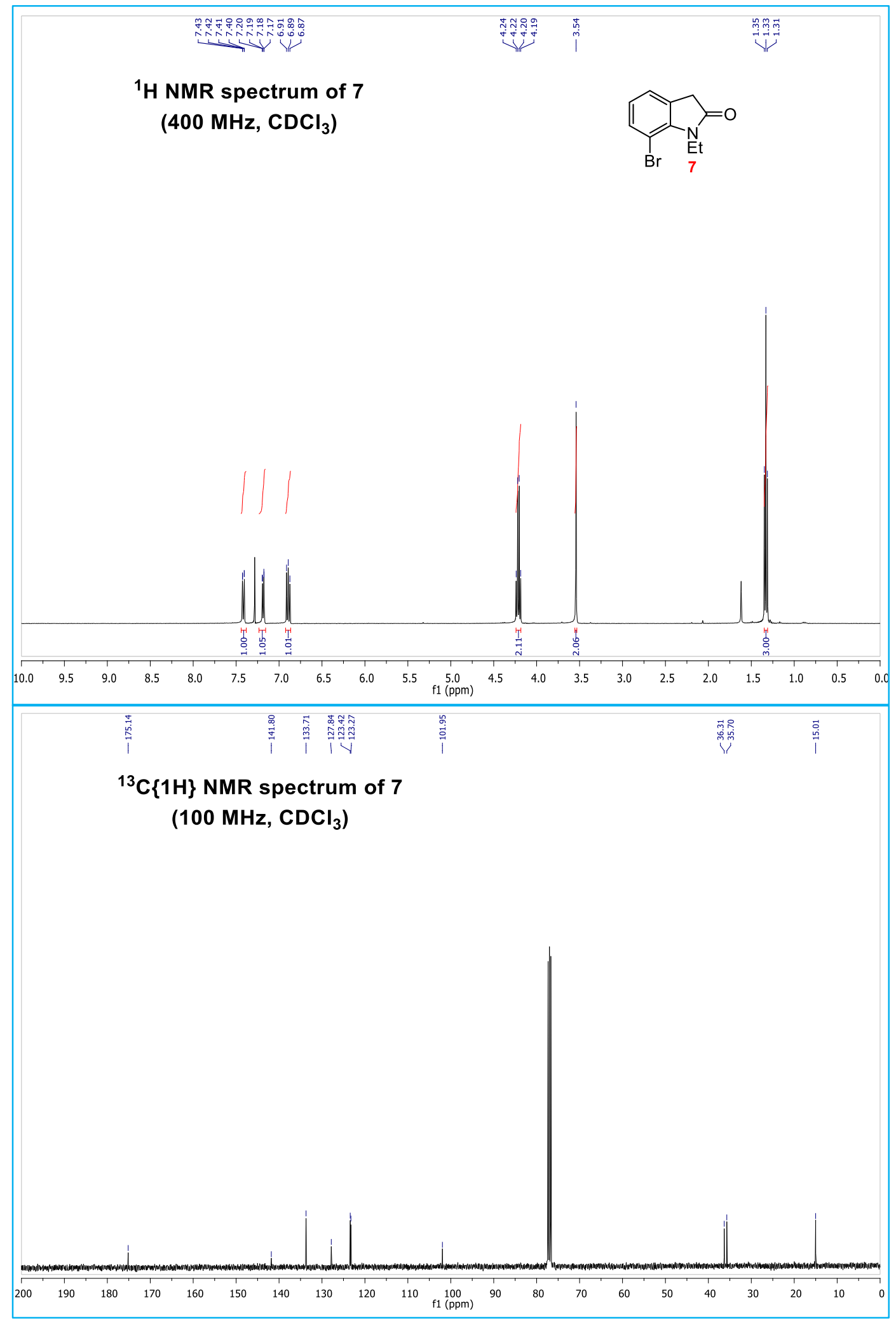


1-Ethyl-7-phenylindolin-2-one (8a)

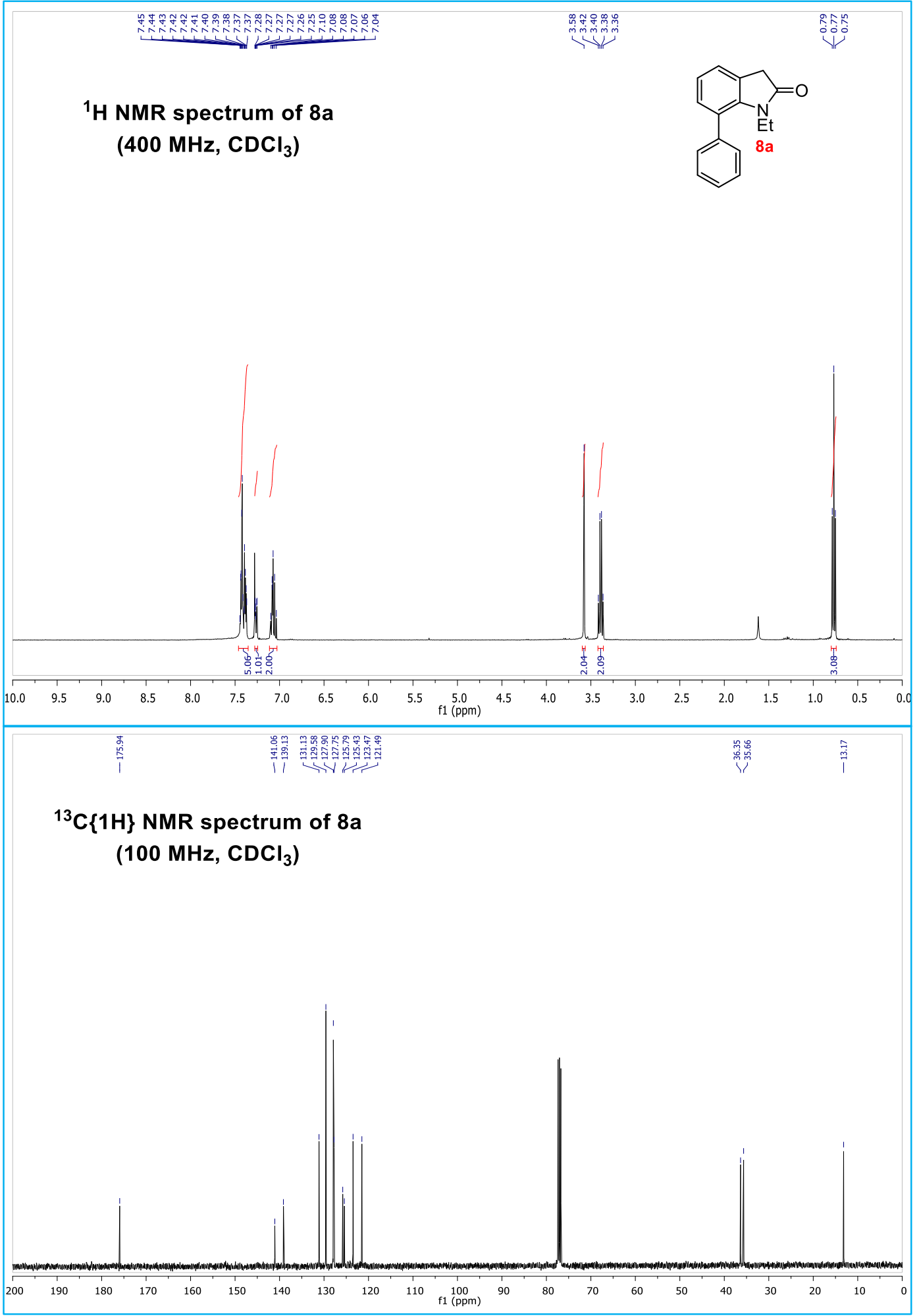


1-Ethyl-7-( $p$-tolyl)indolin-2-one (8b)

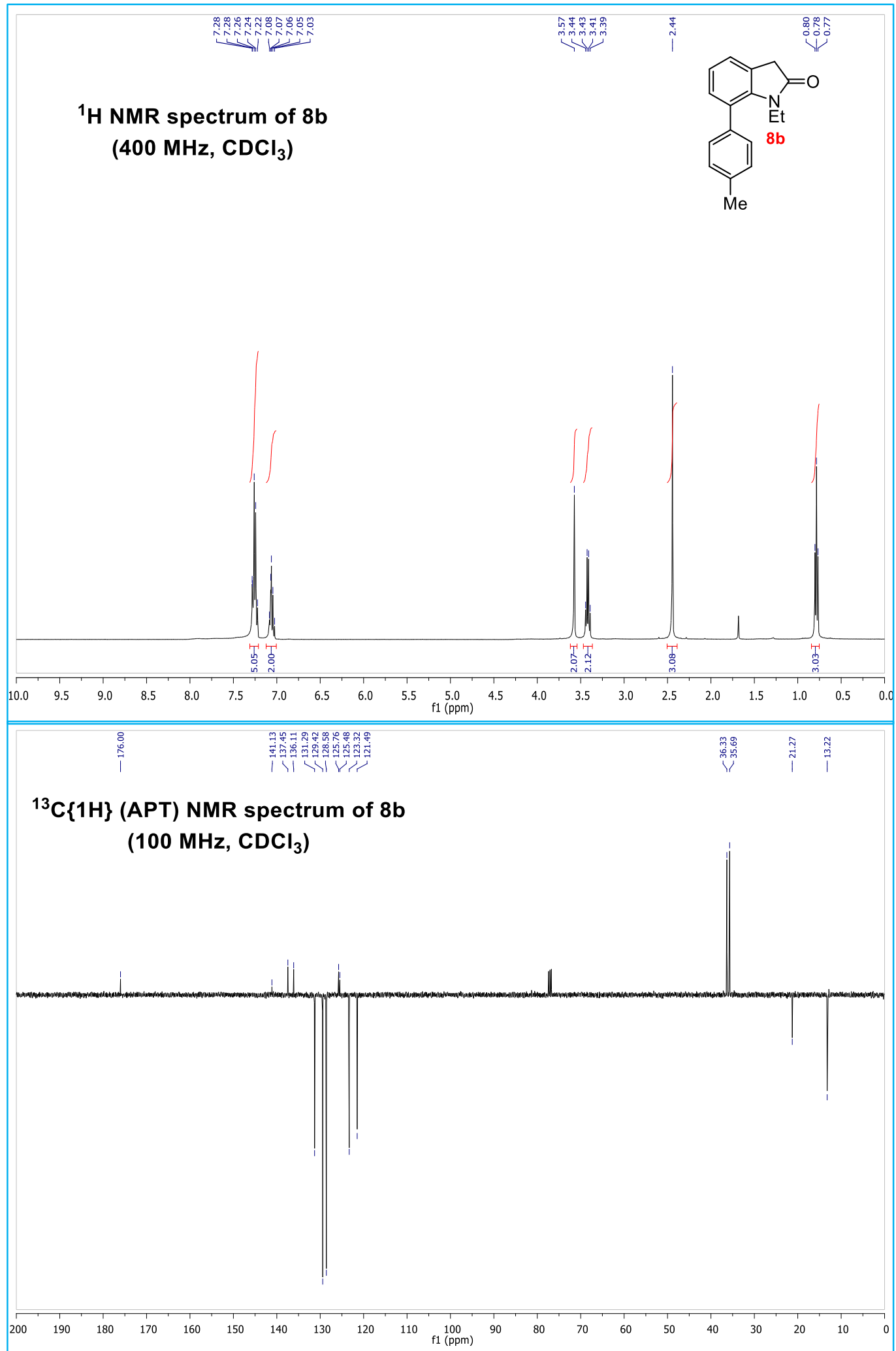


7-(4-(tert-Butyl)phenyl)-1-ethylindolin-2-one (8c)

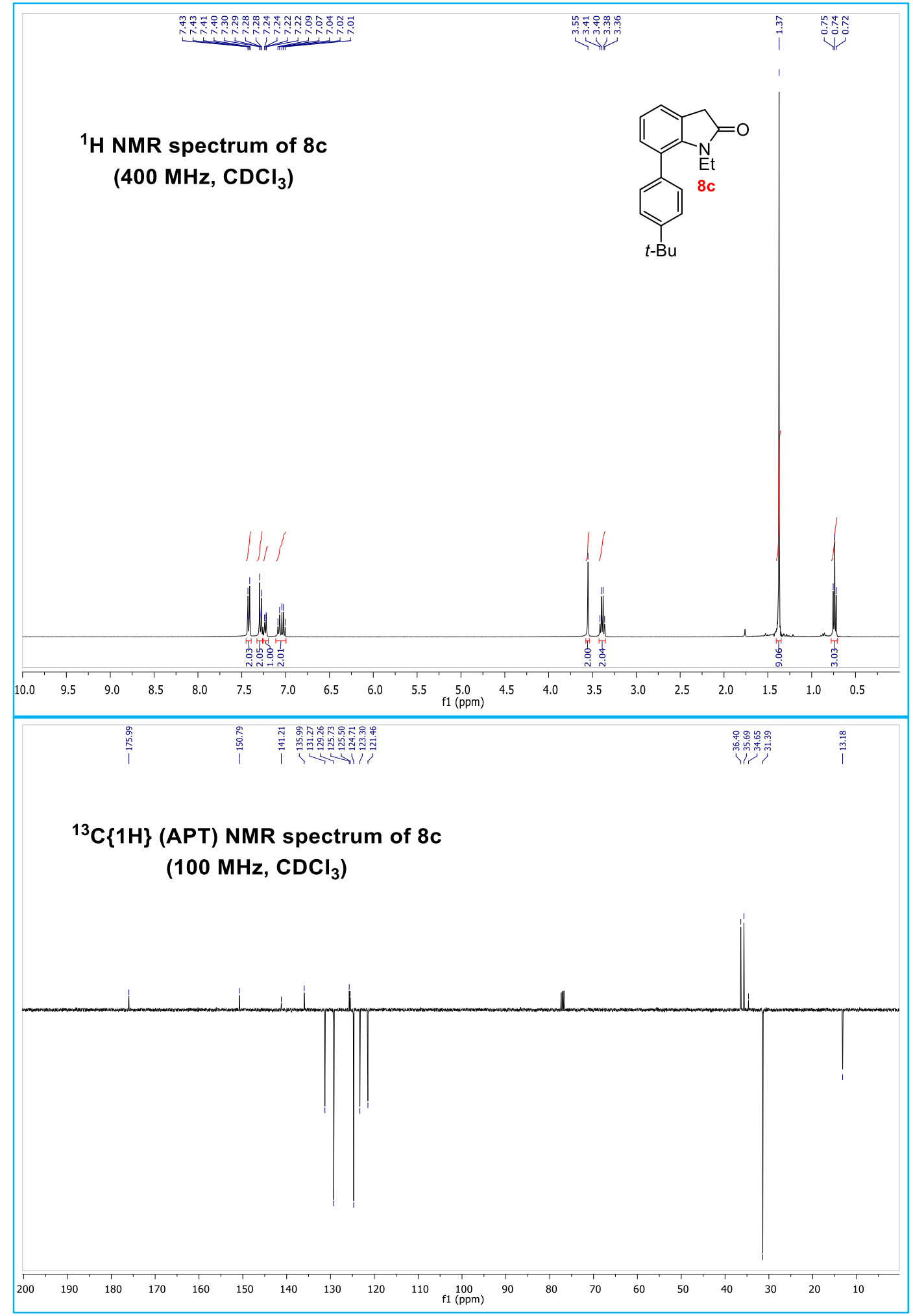


7-([1,1'-Biphenyl]-4-yl)-1-ethylindolin-2-one (8d)

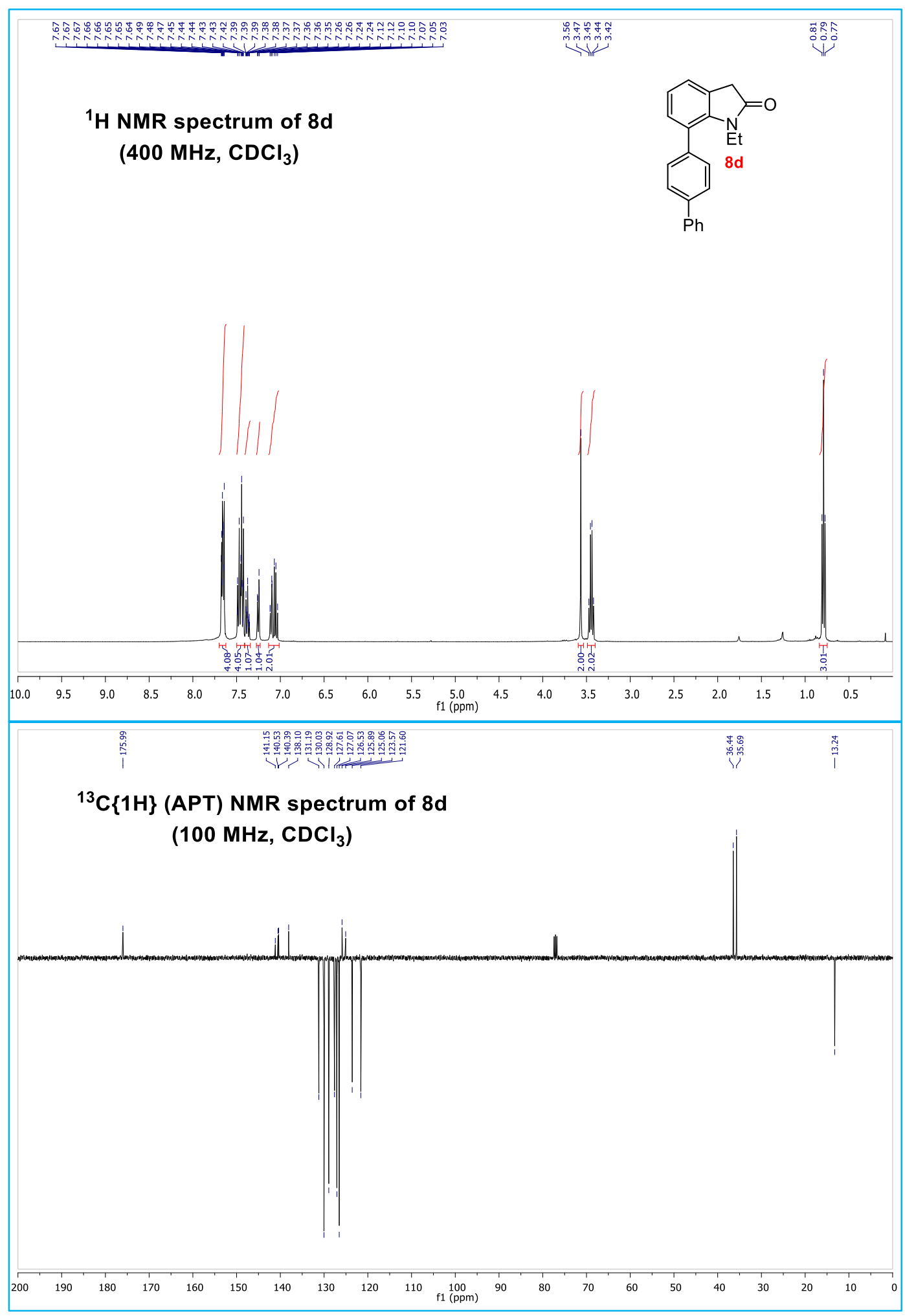


1-Ethyl-7-(4-methoxyphenyl)indolin-2-one (8e)

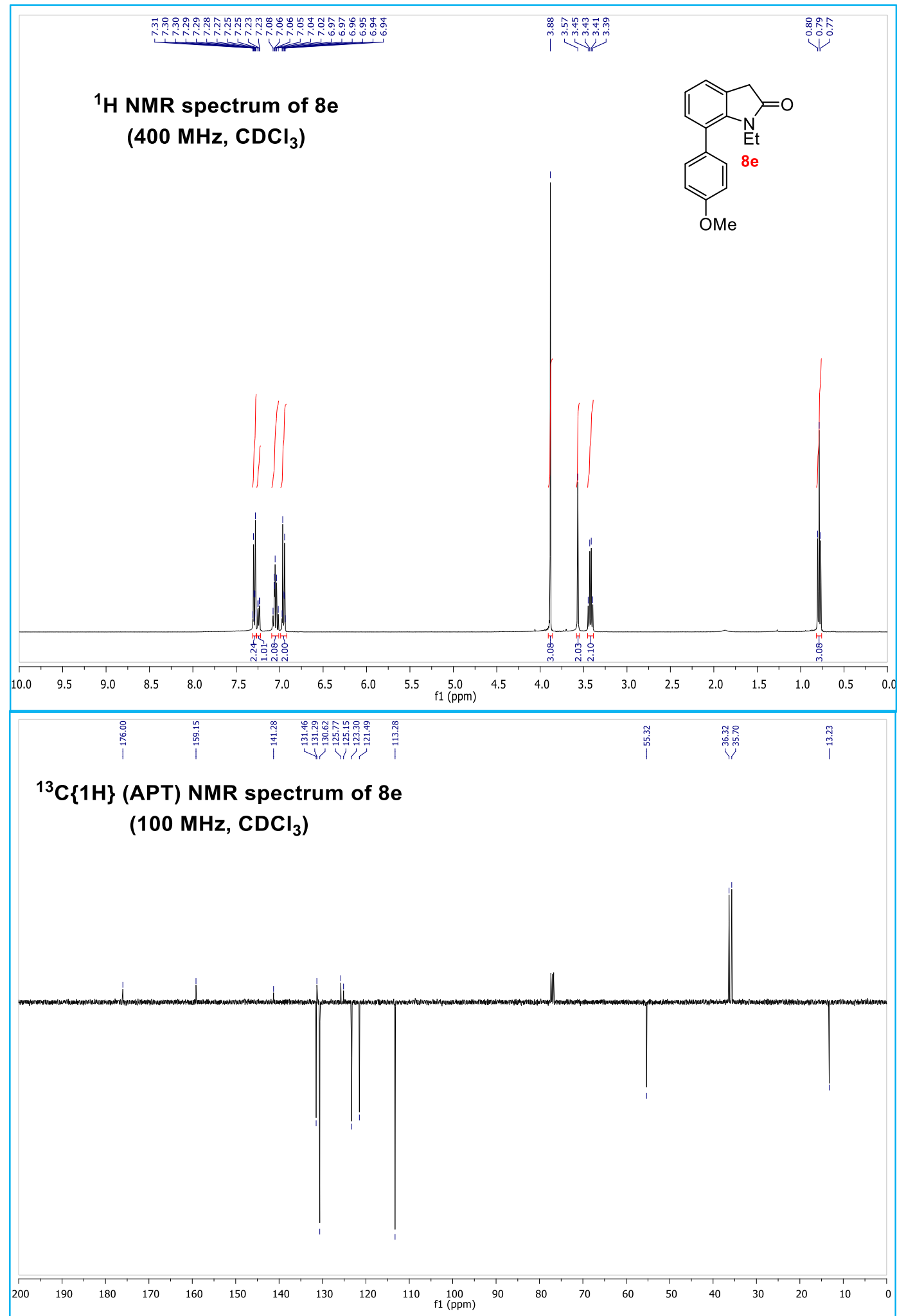




\section{4-(1-Ethyl-2-oxoindolin-7-yl)benzonitrile (8f)}

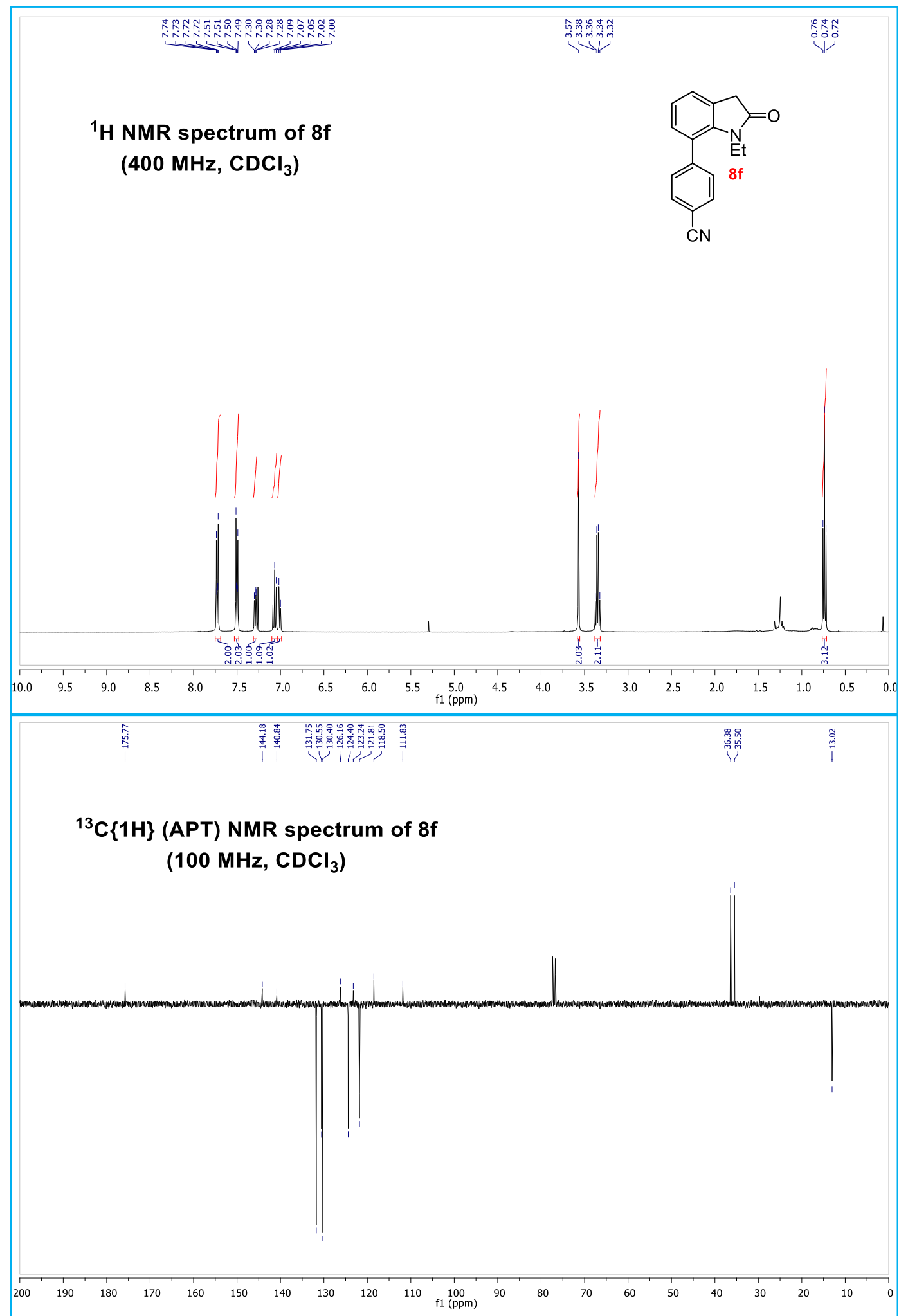


1-Ethyl-7-(4-(trifluoromethyl)phenyl)indolin-2-one (8g)

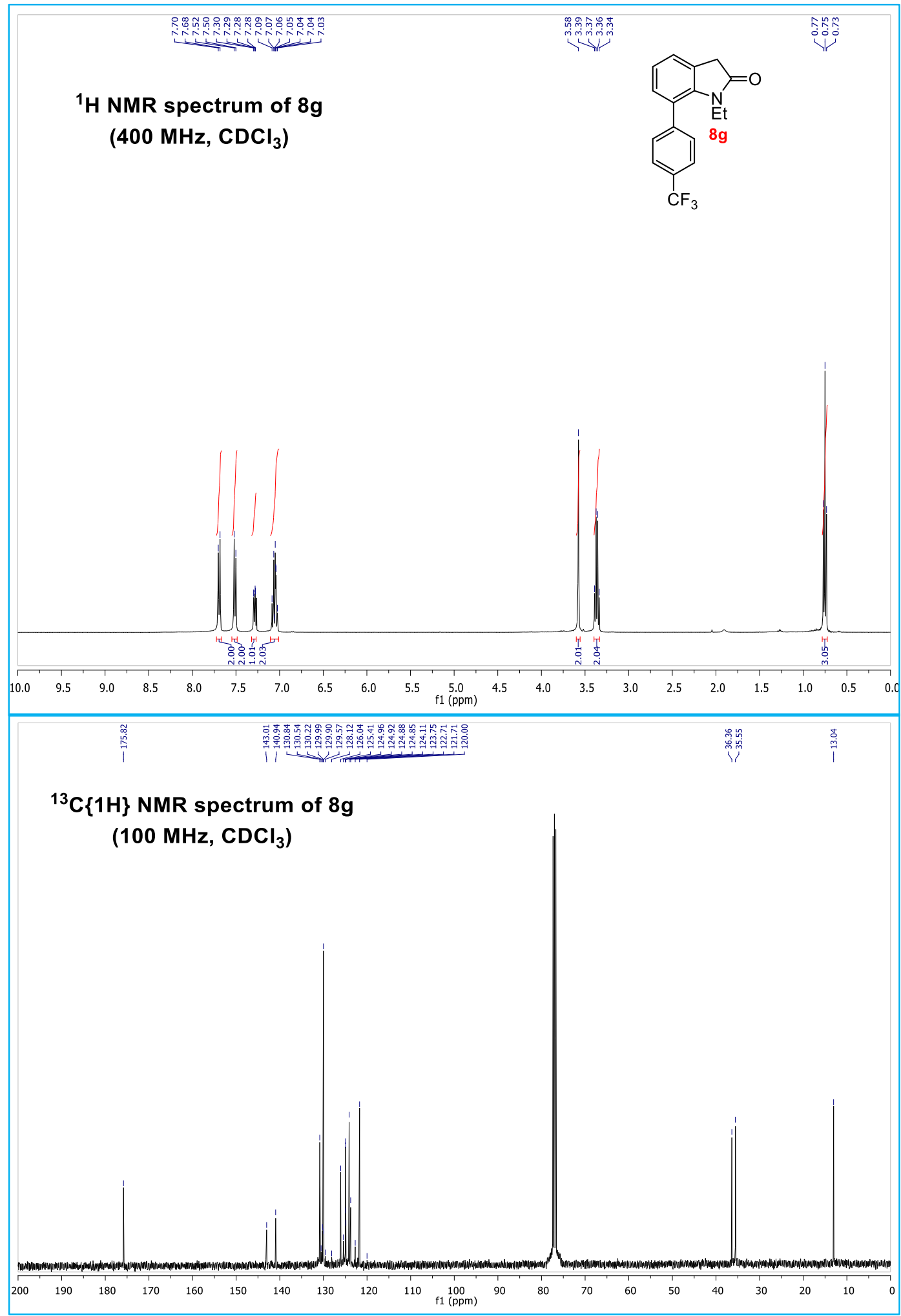


1-Ethyl-7-(4-fluorophenyl)indolin-2-one (8h)
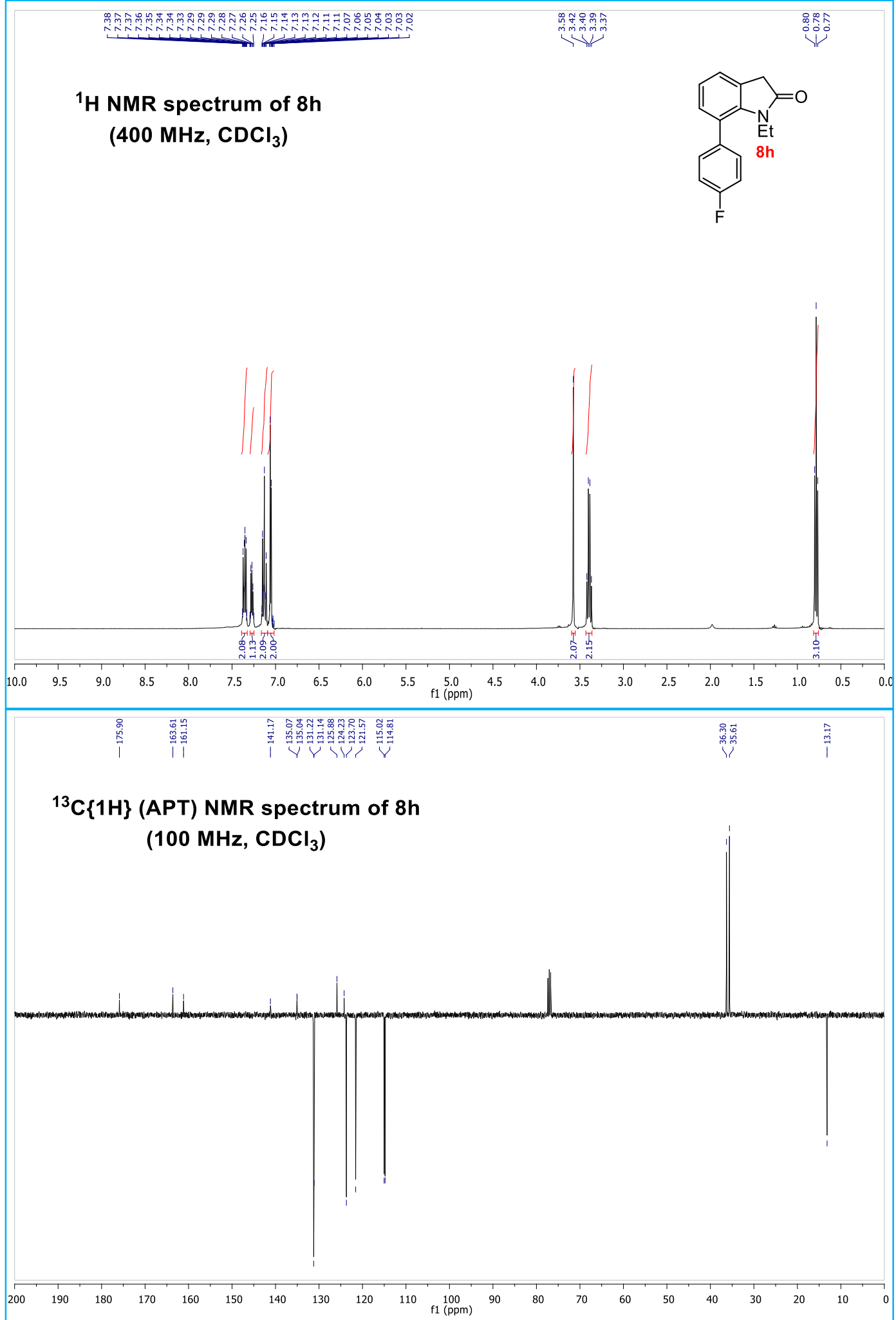
( \pm )-1-Ethyl-7-(o-tolyl)indolin-2-one (8j)

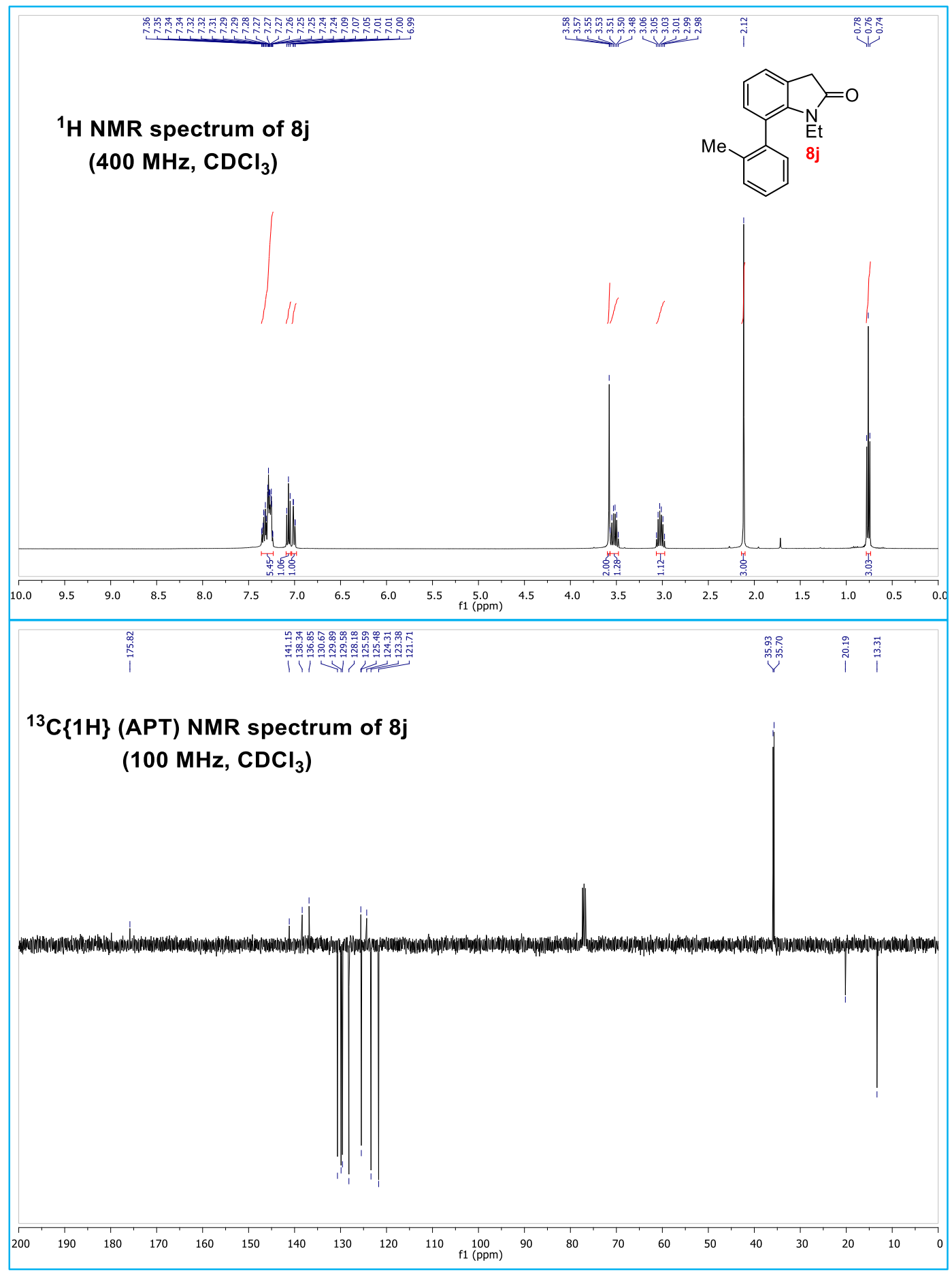


7-(3,4-Dimethylphenyl)-1-ethylindolin-2-one (8k)

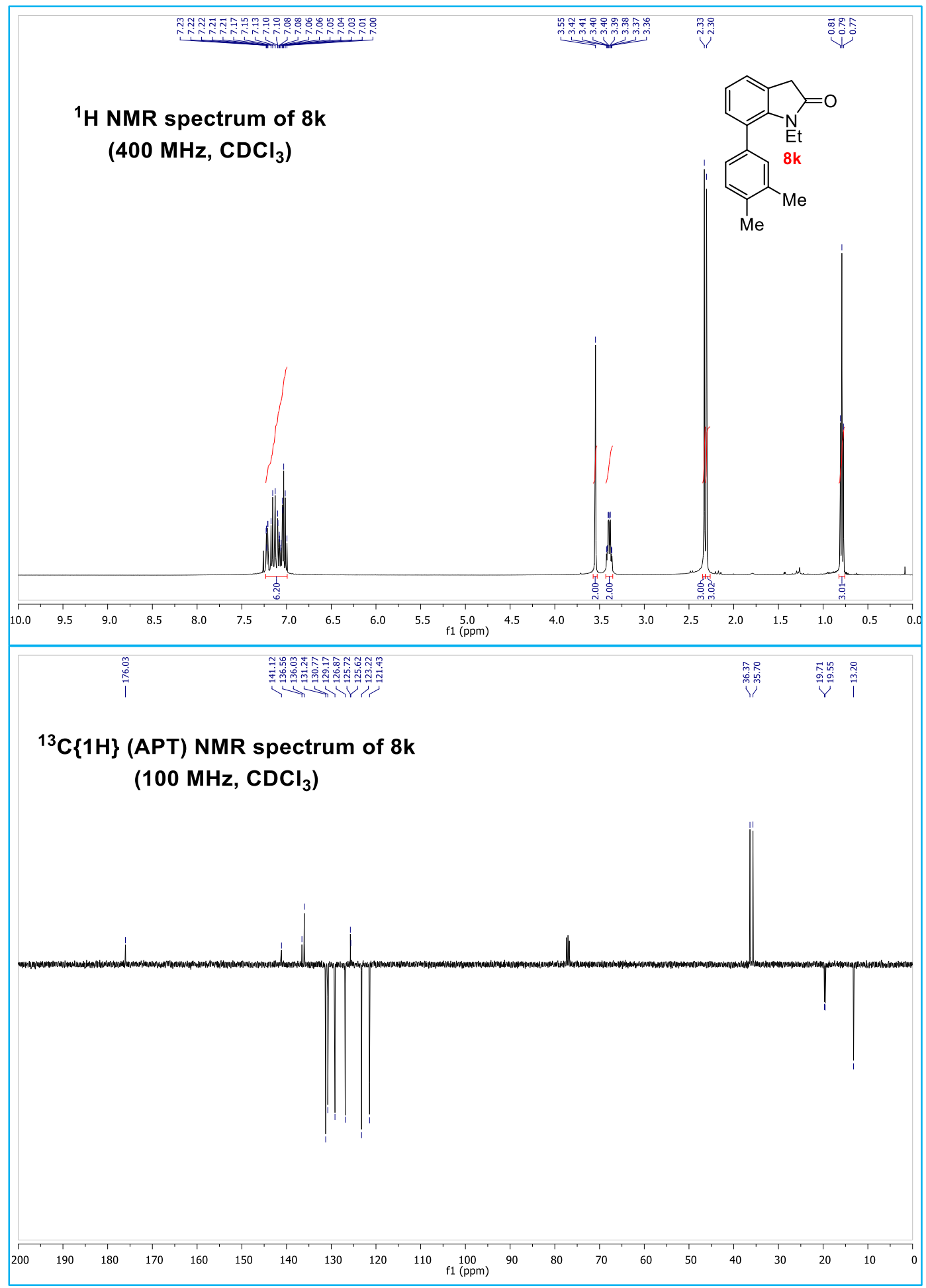


1-Ethyl-7-(3-nitrophenyl)indolin-2-one (81)

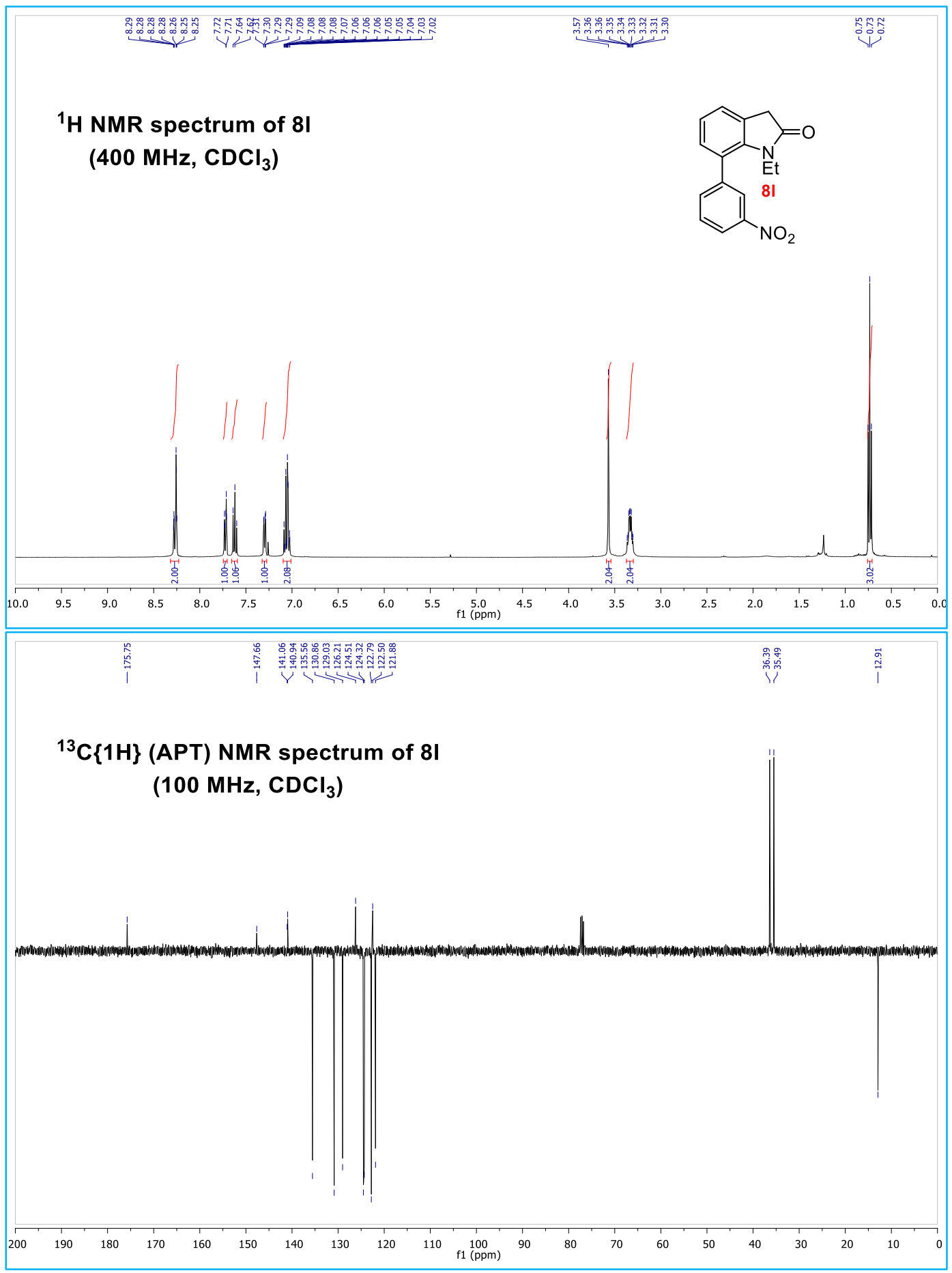


1-Ethyl-7-(3-methoxyphenyl)indolin-2-one (8m)

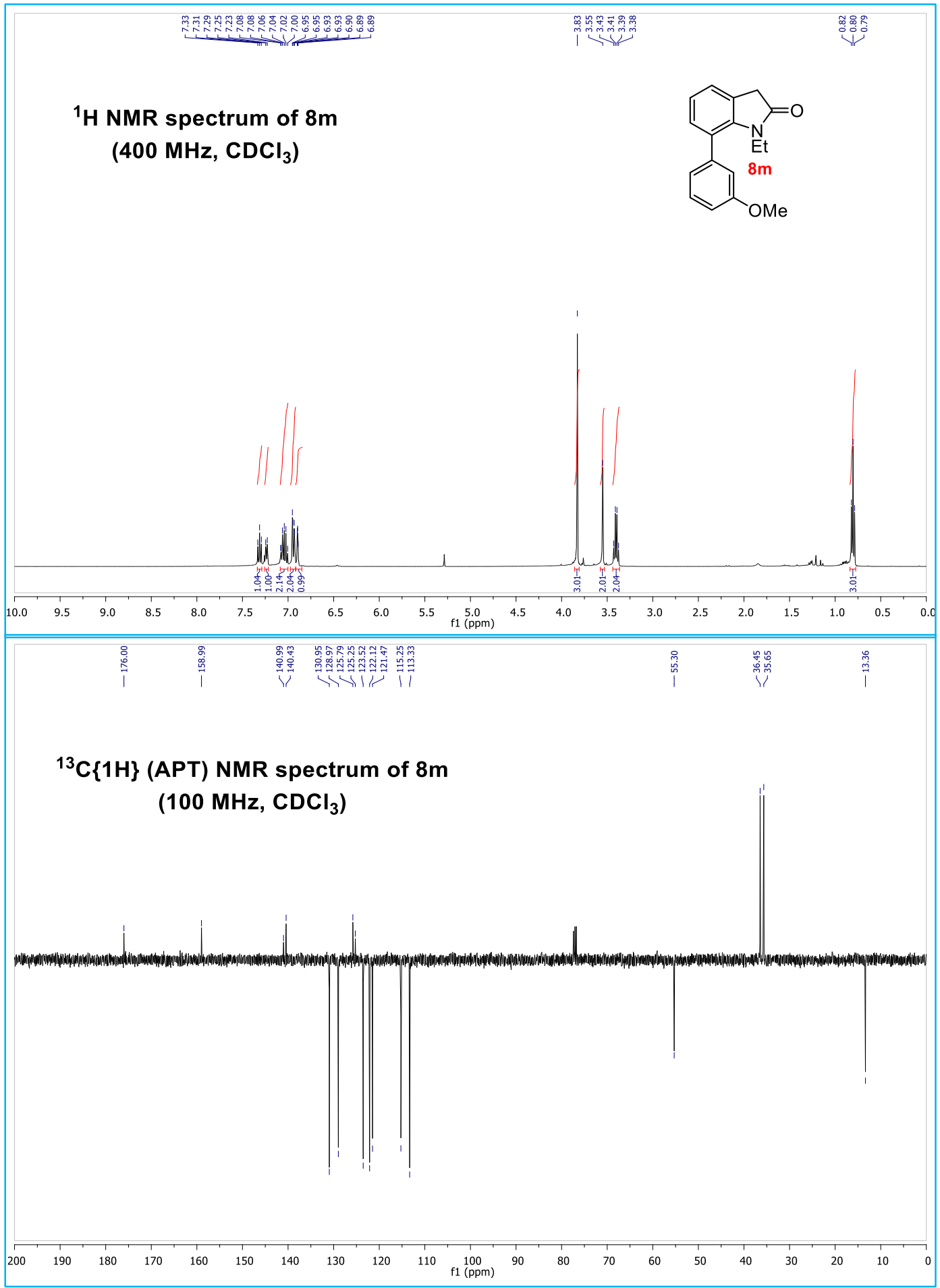


1-Ethyl-7-(m-tolyl)indolin-2-one (8n)

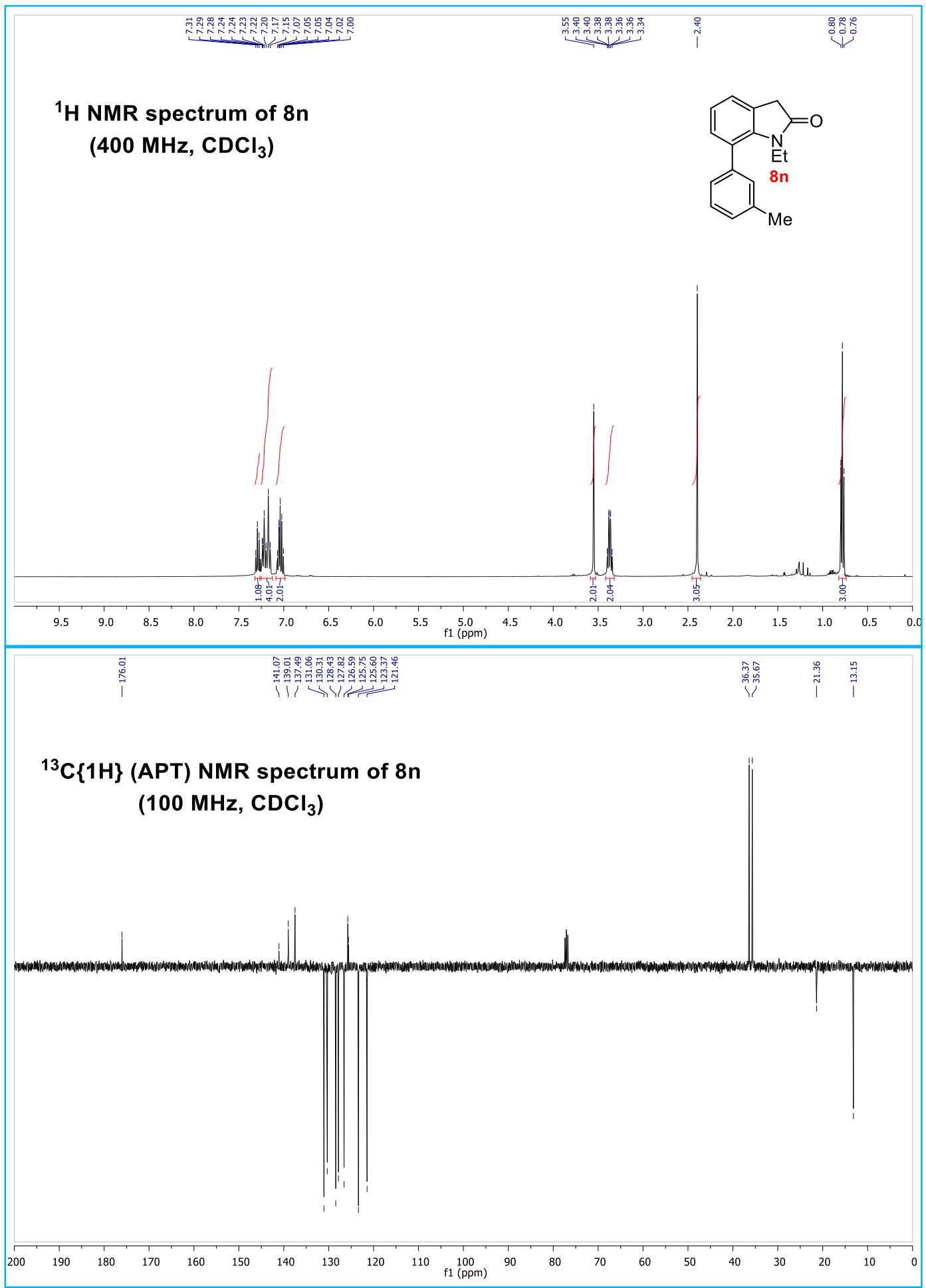


1-Ethyl-7-(pyridin-4-yl)indolin-2-one (8o)

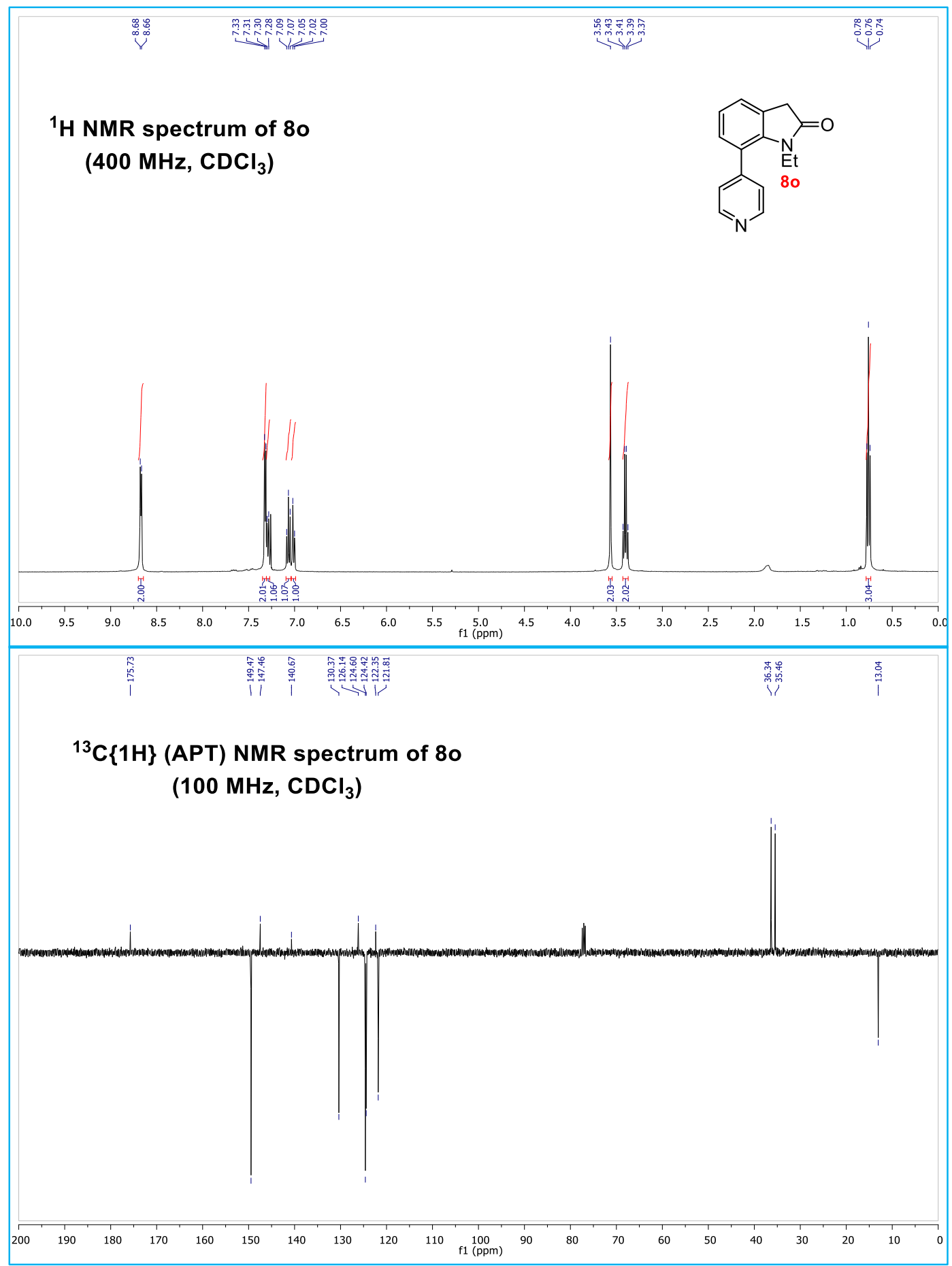


1-Ethyl-7-(thiophen-2-yl)indolin-2-one (8p)

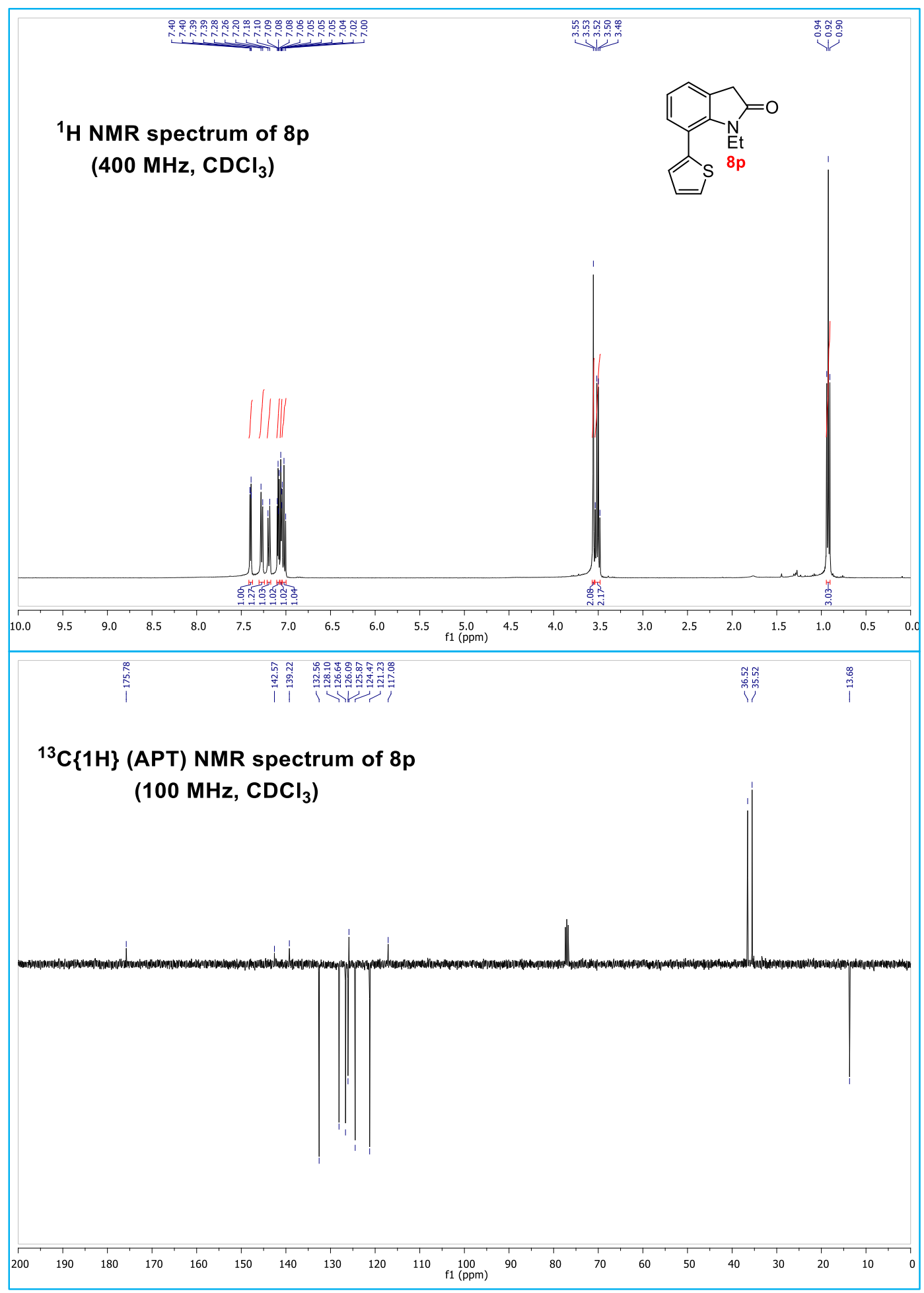


5,10,15-Triethyl-1,6,11-triphenyl-10,15-dihydro-5H-diindolo[3,2-a:3',2'-c]carbazole (9a)

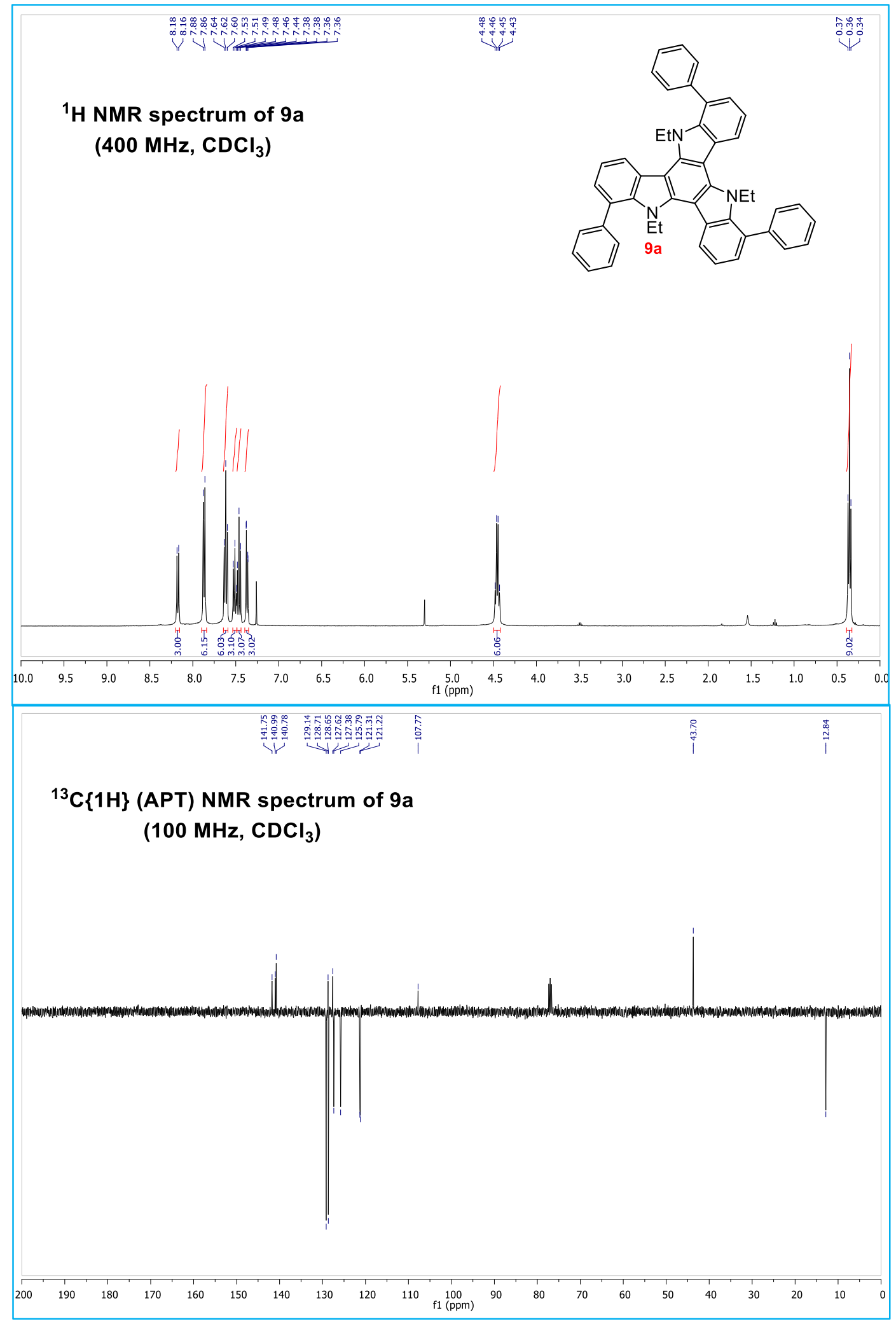


5,10,15-Triethyl-1,6,11-tri- $p$-tolyl-10,15-dihydro-5H-diindolo[3,2-a:3',2'-c]carbazole (9b)

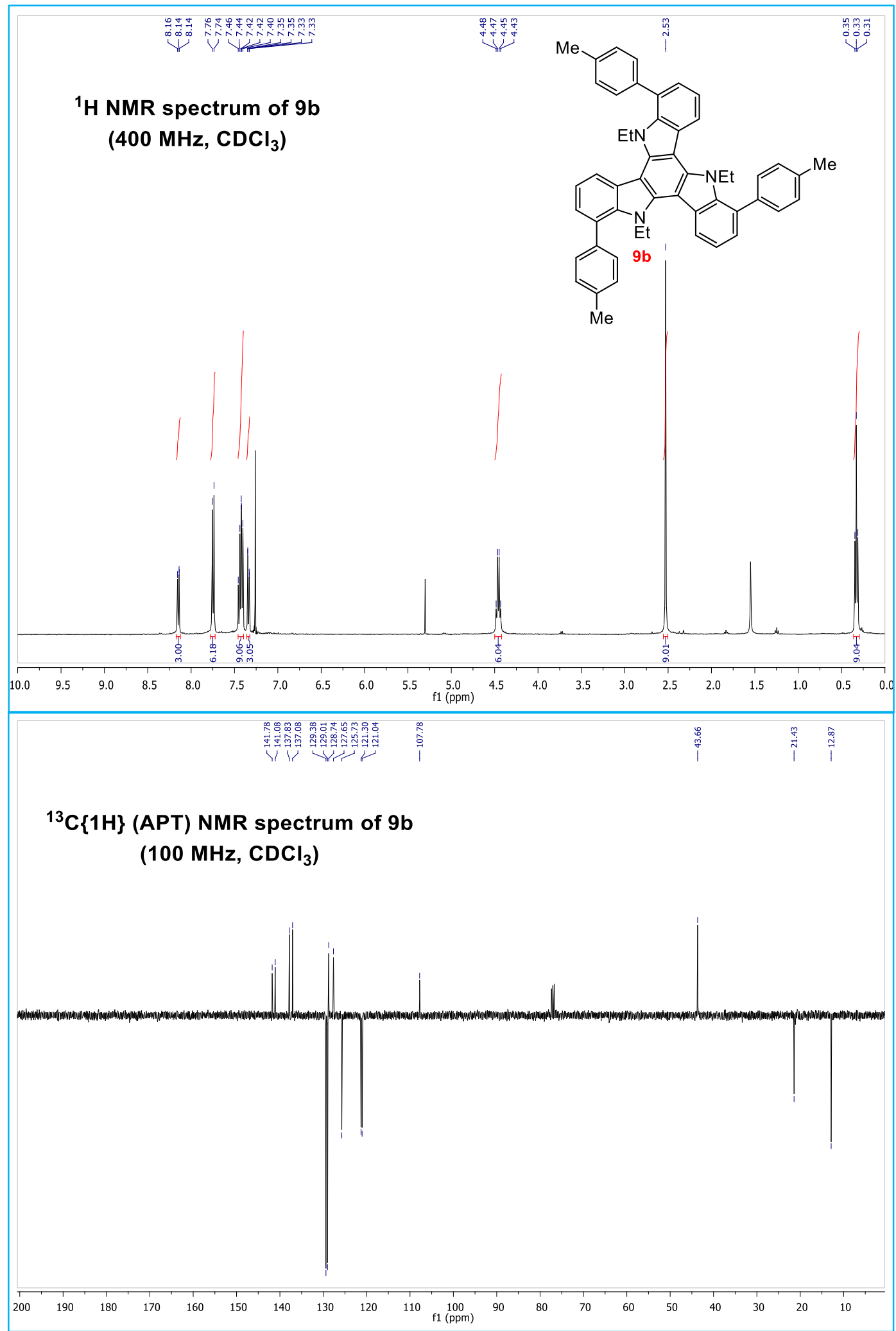


1,6,11-Tris(4-(tert-butyl)phenyl)-5,10,15-triethyl-10,15-dihydro-5H-diindolo[3,2-a:3',2'c] carbazole (9c)

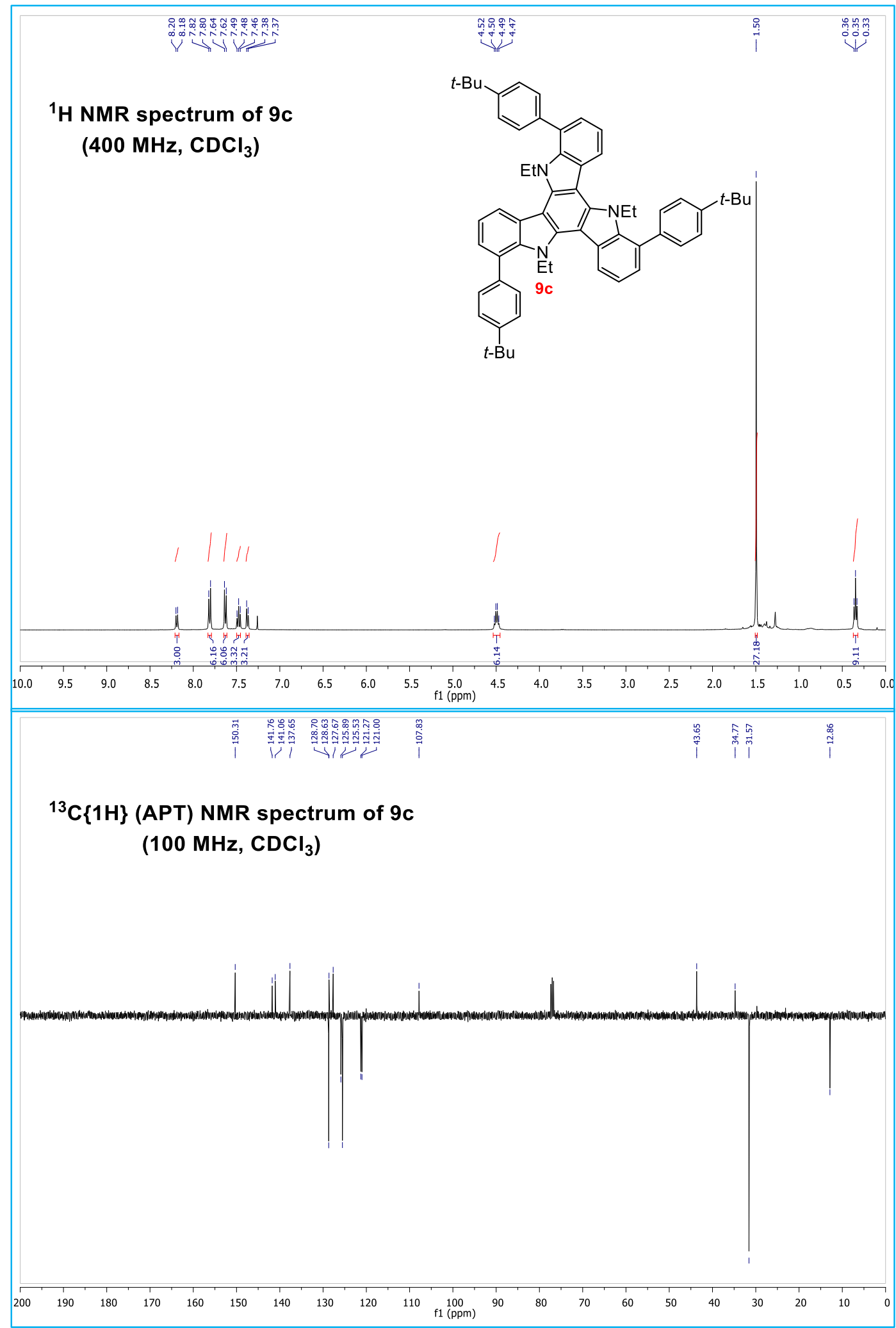


1,6,11-Tri([1,1'-biphenyl]-4-yl)-5,10,15-triethyl-10,15-dihydro-5H-diindolo[3,2-a:3',2'c]carbazole (9d)

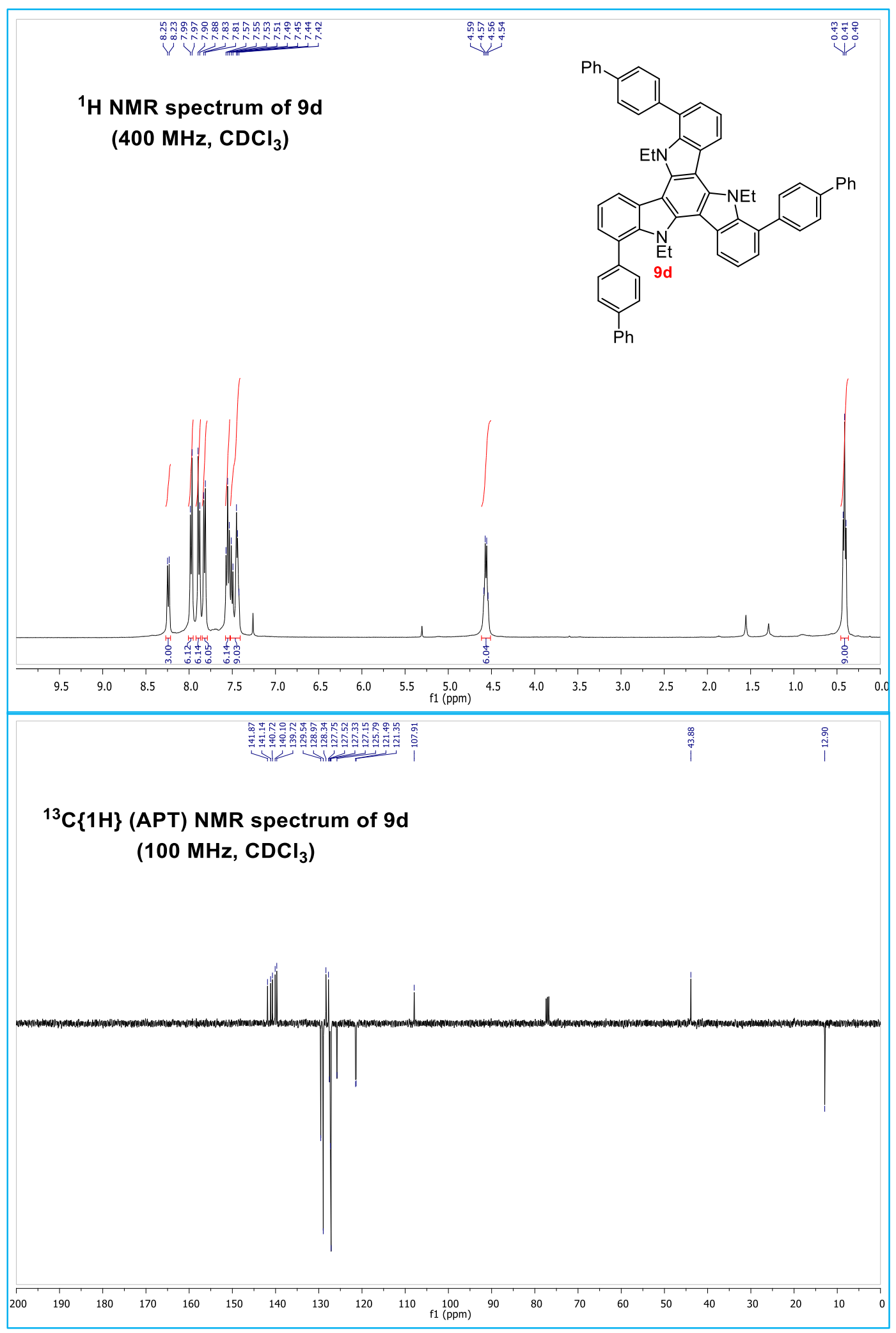


2-Chloro-1-ethyl-7-(4-methoxyphenyl)-1H-indole (11e)

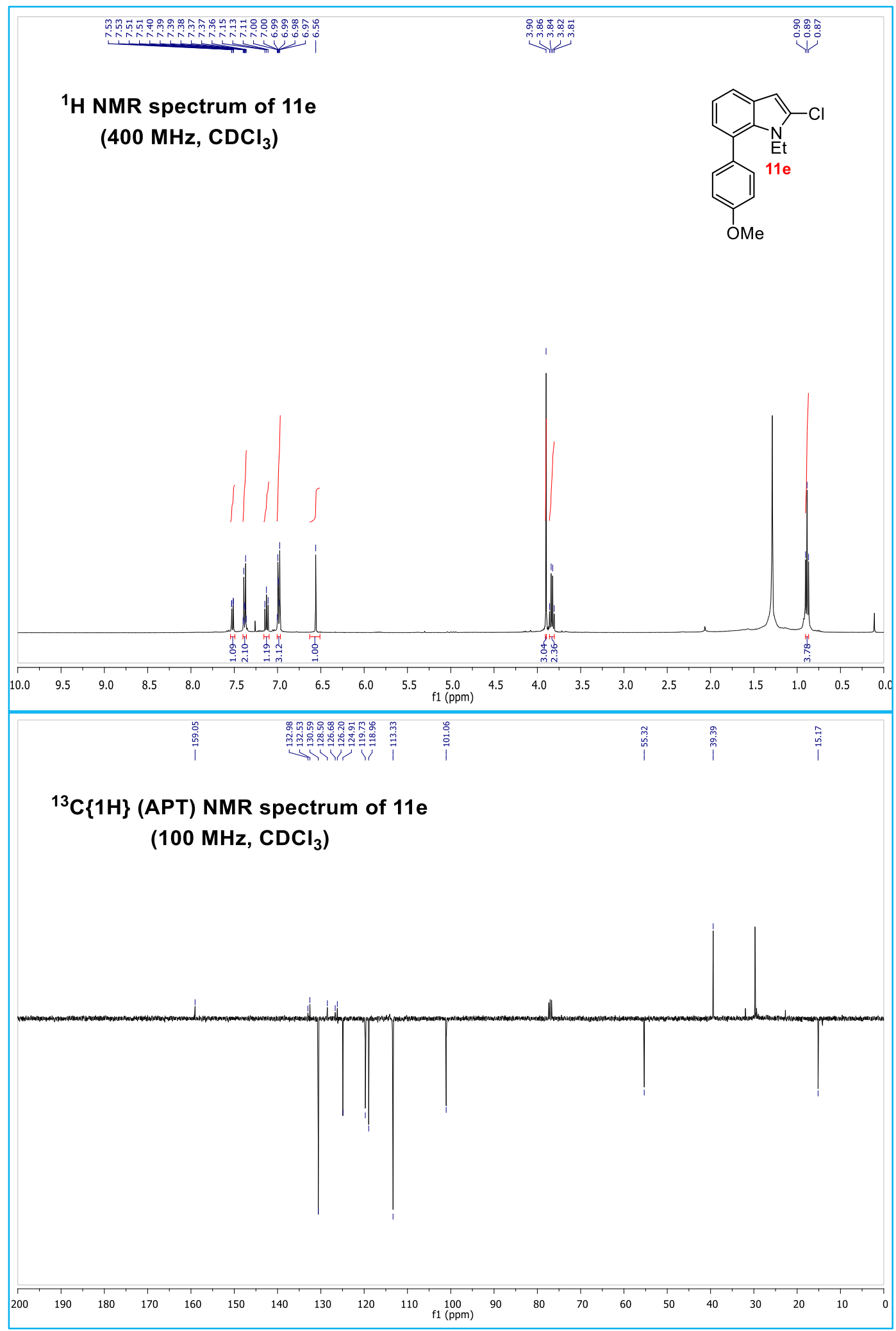


5,10,15-Triethyl-1,6,11-tris(4-methoxyphenyl)-10,15-dihydro-5H-diindolo[3,2-a:3',2'c]carbazole (9e)

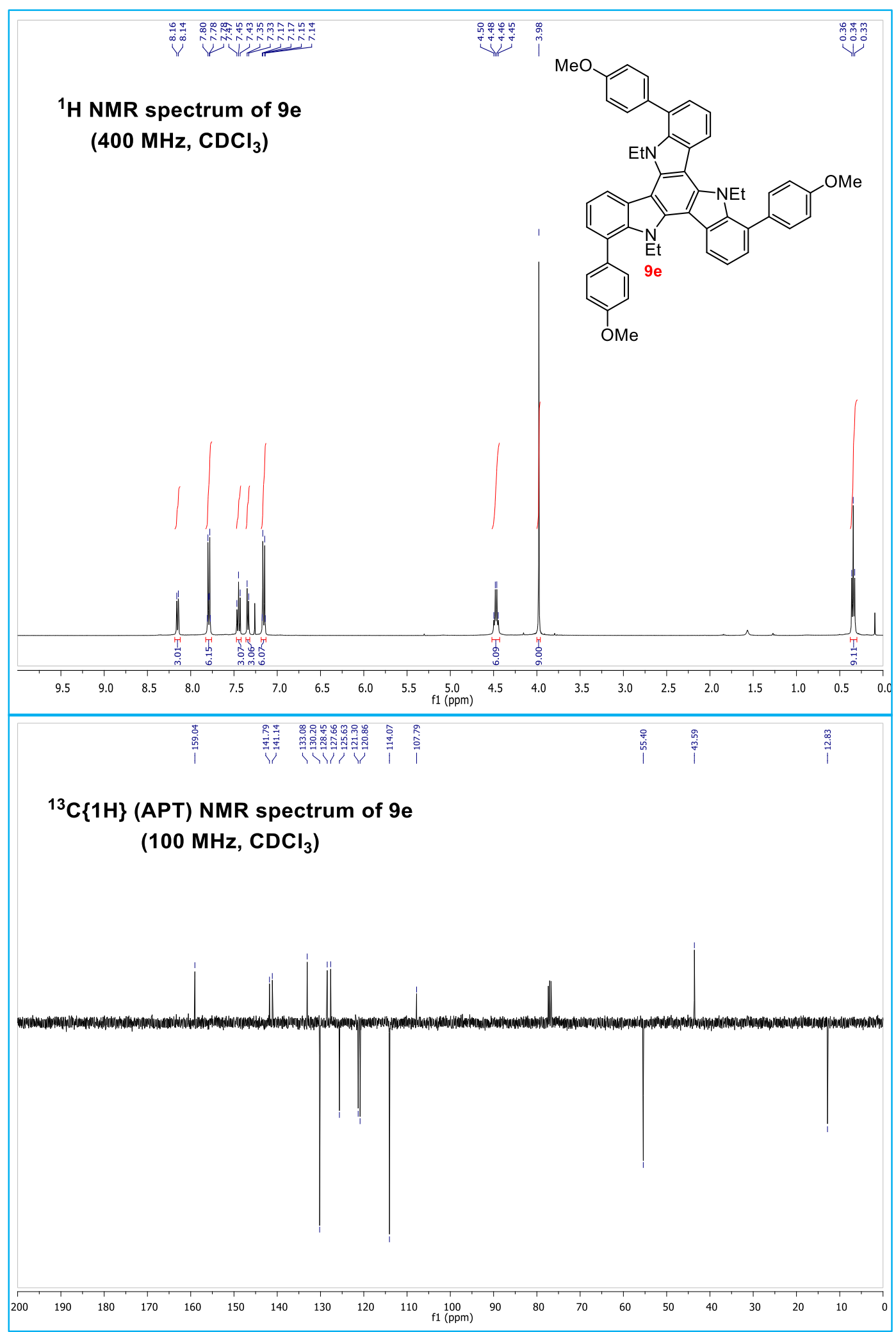




\section{4-(2-Chloro-1-ethyl-1H-indol-7-yl)benzonitrile (11f)}

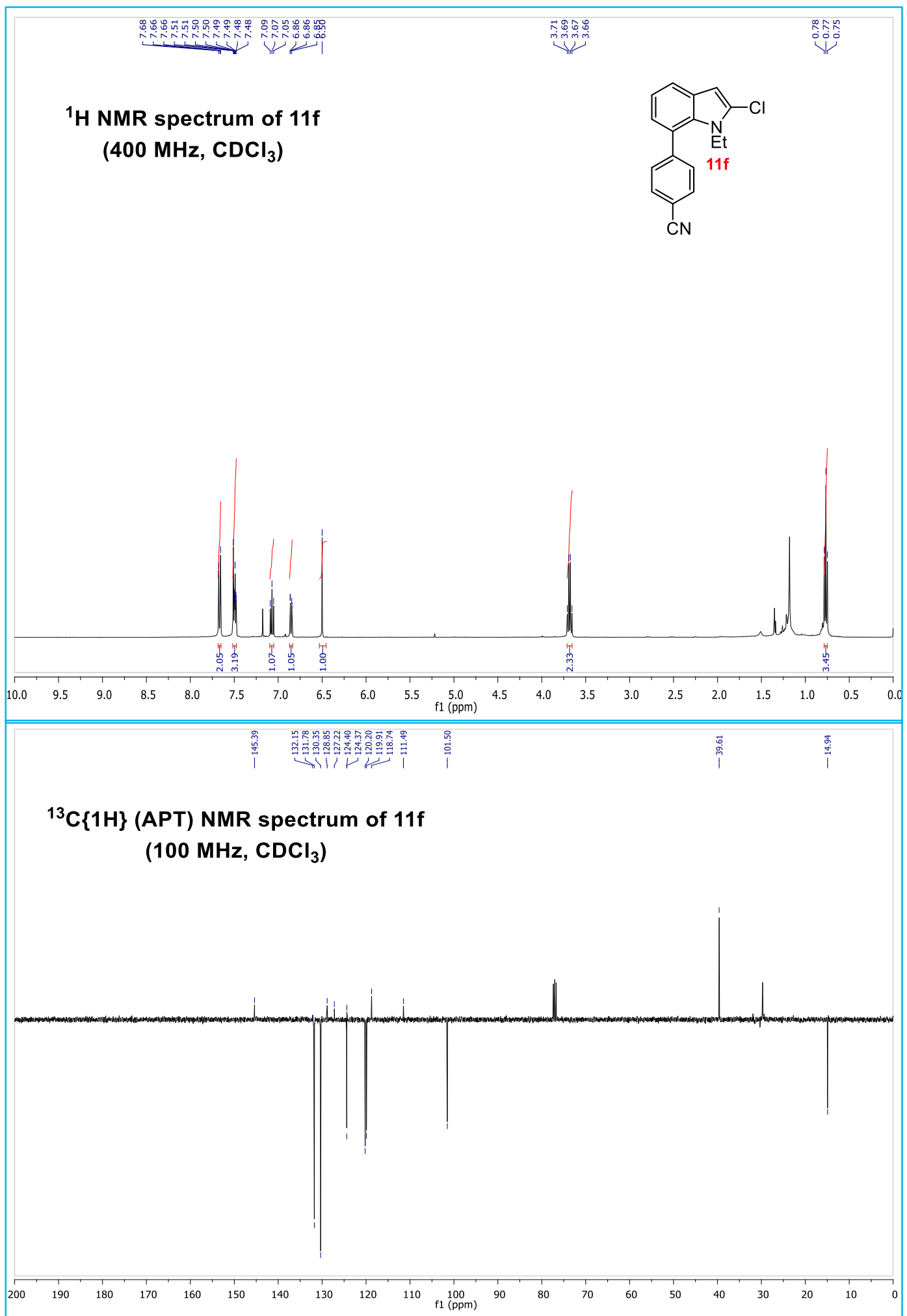


2-Chloro-1-ethyl-7-(4-(trifluoromethyl)phenyl)-1H-indole (11g)

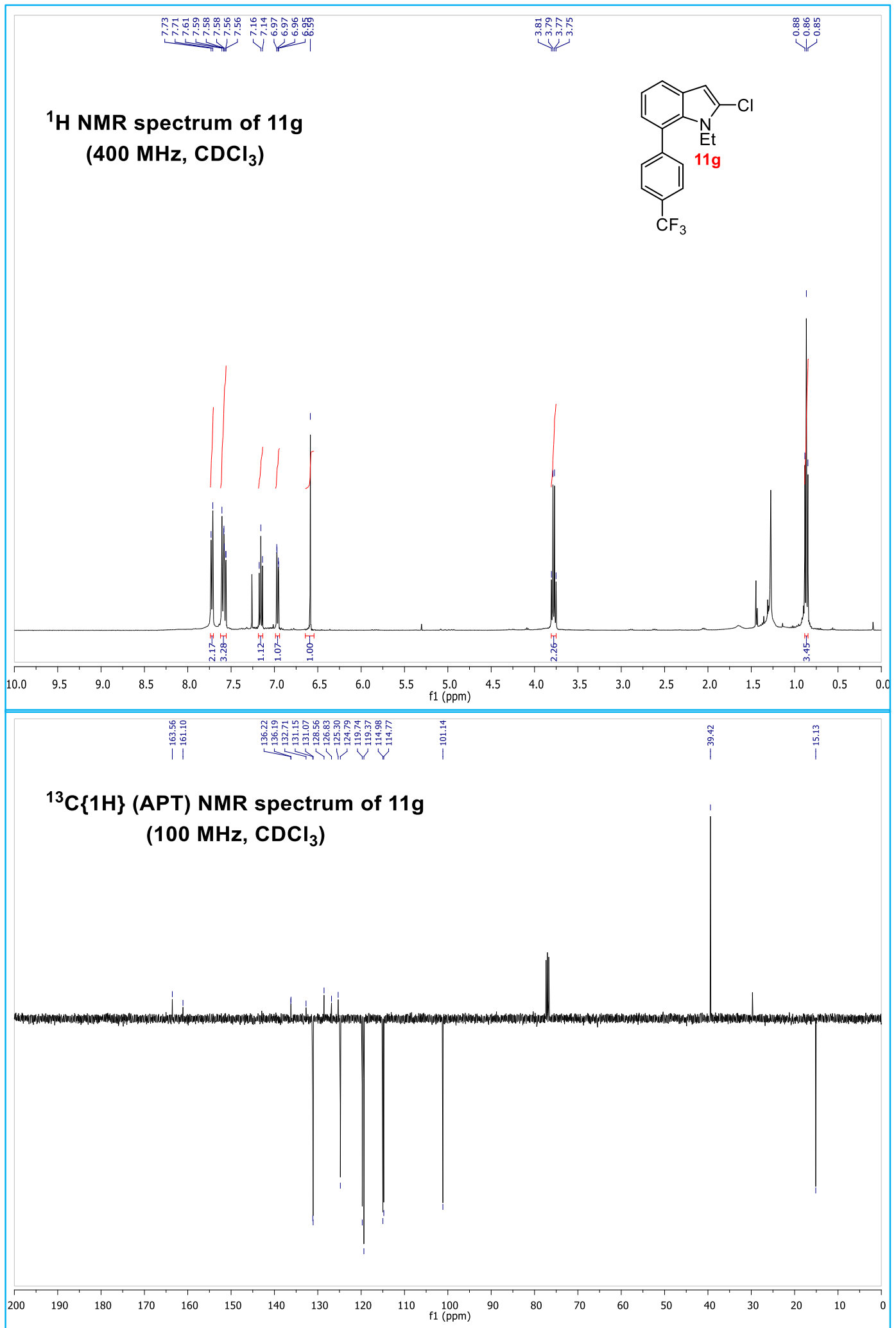


2-Chloro-1-ethyl-7-(4-fluorophenyl)-1H-indole (11h)

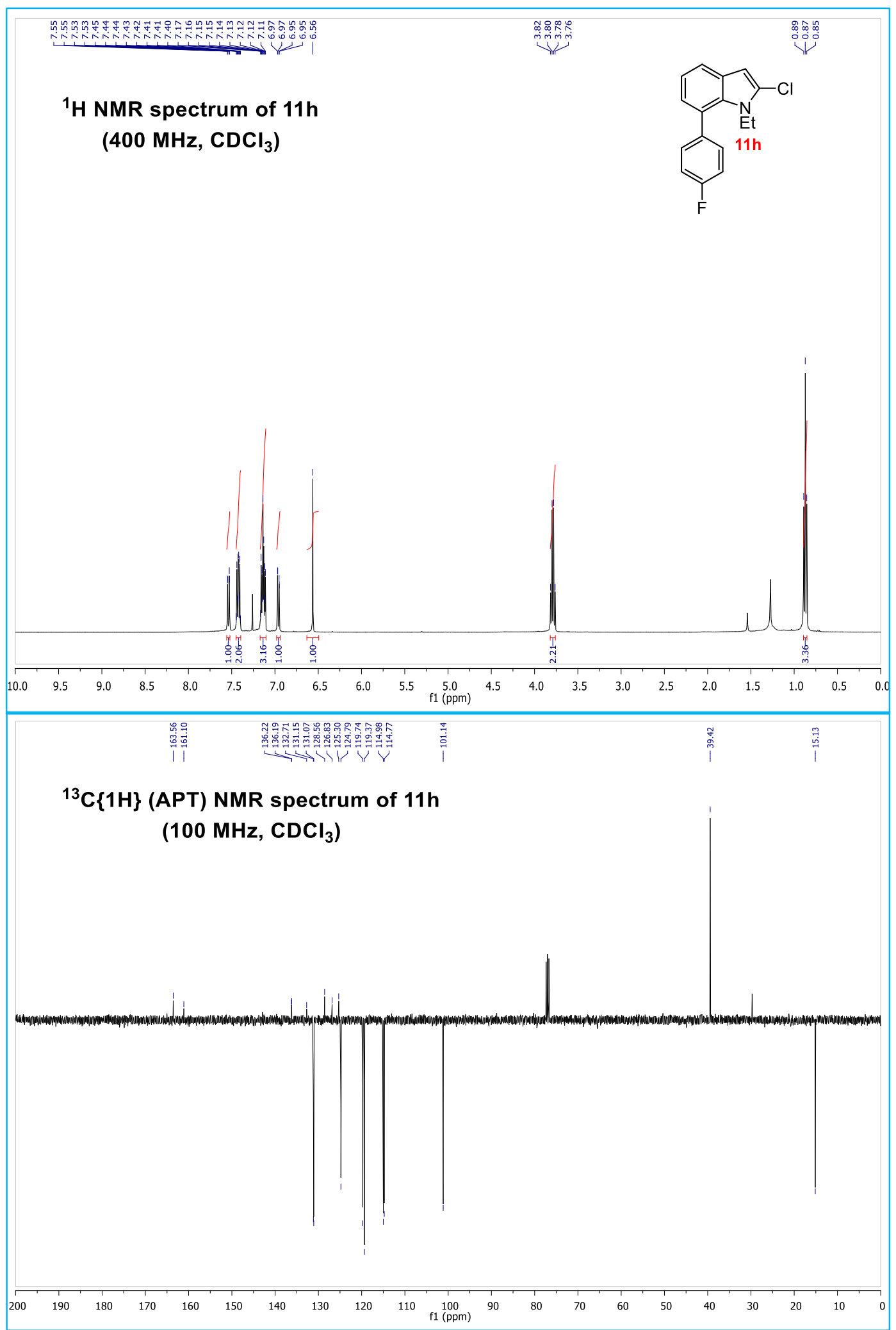


5,10,15-Triethyl-1,6,11-tri-o-tolyl-10,15-dihydro-5H-diindolo[3,2-a:3',2'-c]carbazole $\quad(9 \mathrm{j}-\mathrm{A}$ and $9 \mathrm{j}-\mathrm{B}$ ) as a mixture of atropisomers

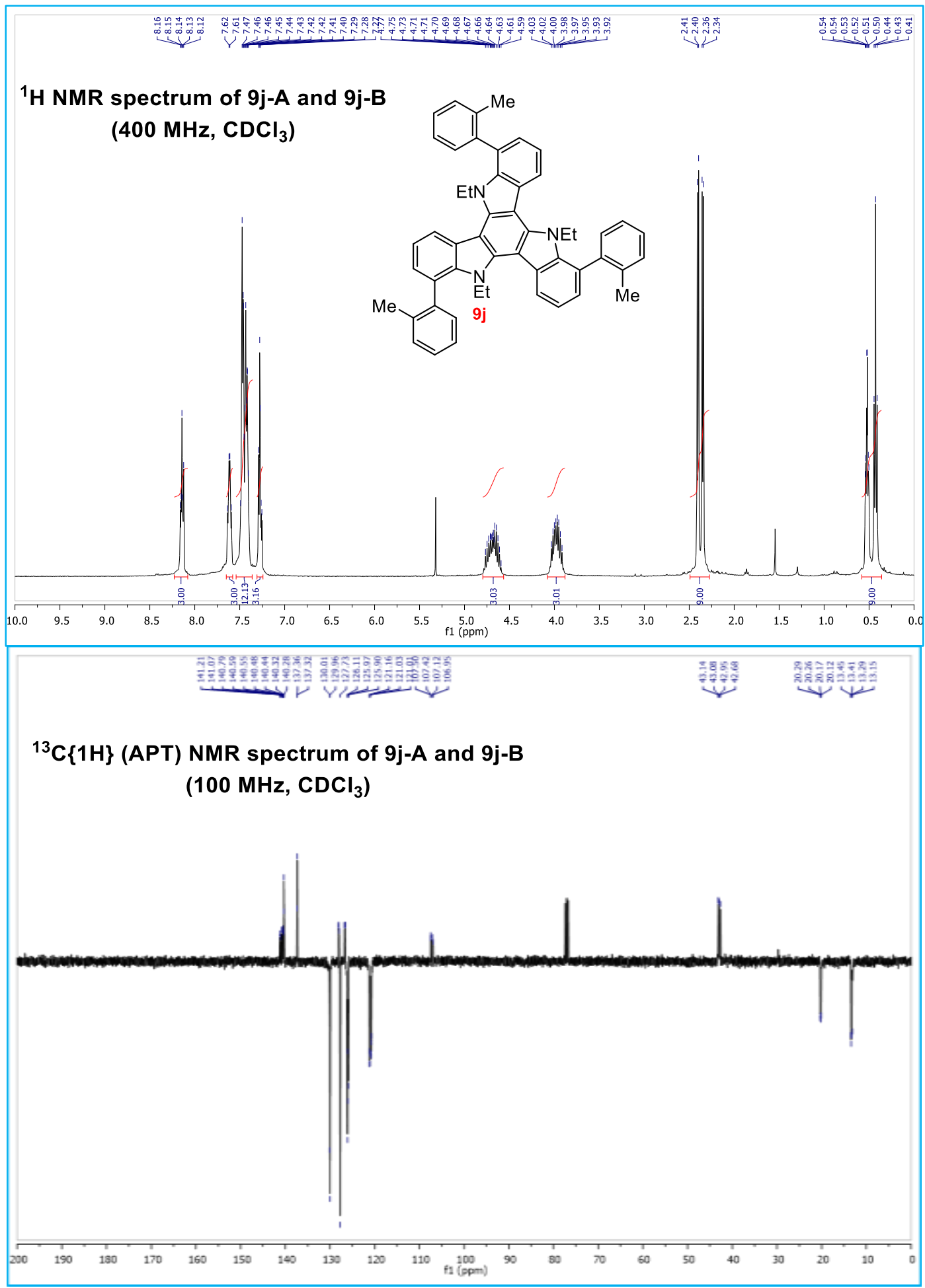


1,6,11-Tris(3,4-dimethylphenyl)-5,10,15-triethyl-10,15-dihydro-5H-diindolo[3,2-a:3',2'c]carbazole (9k)

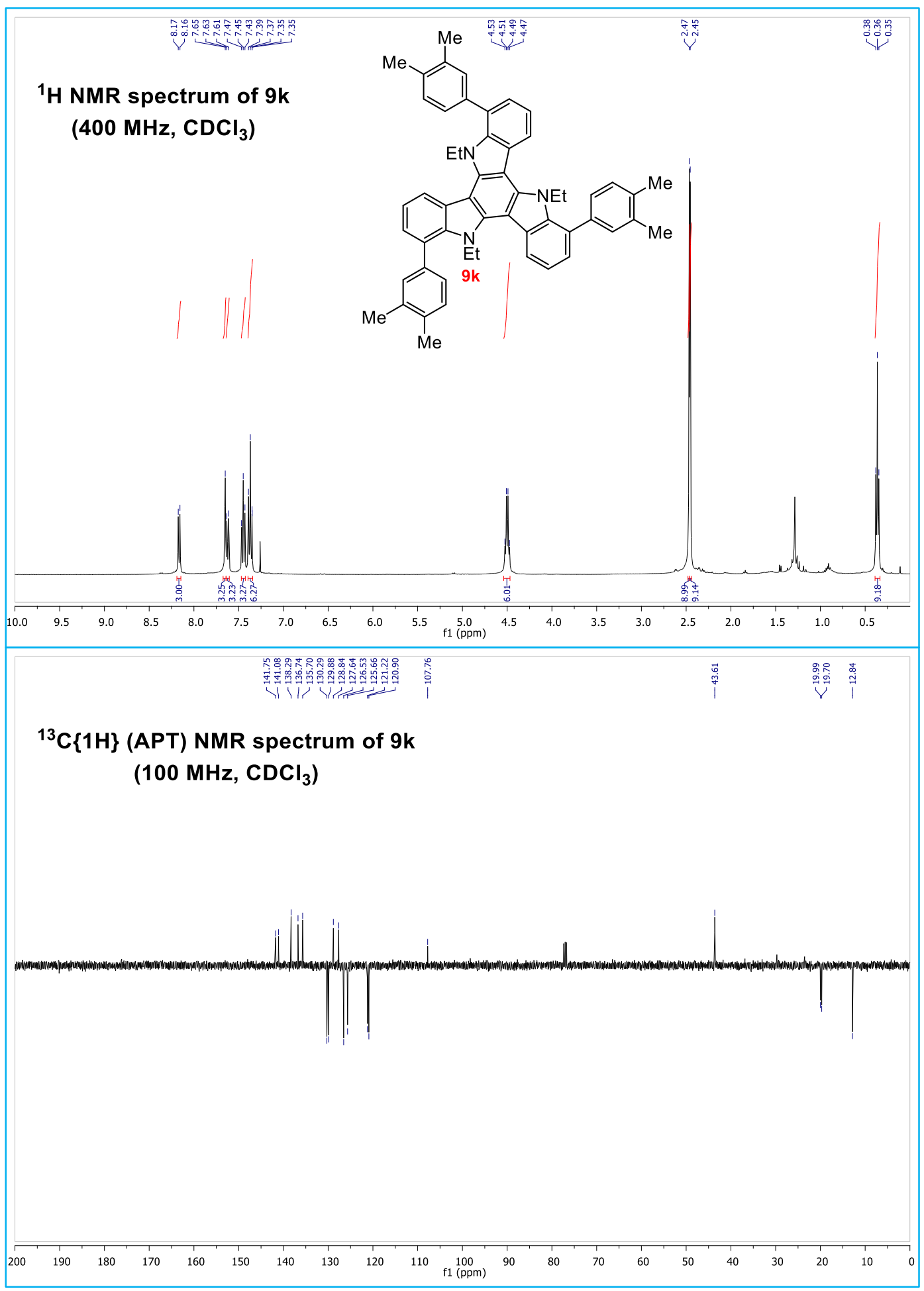


2-Chloro-1-ethyl-7-(3-nitrophenyl)-1H-indole (111)

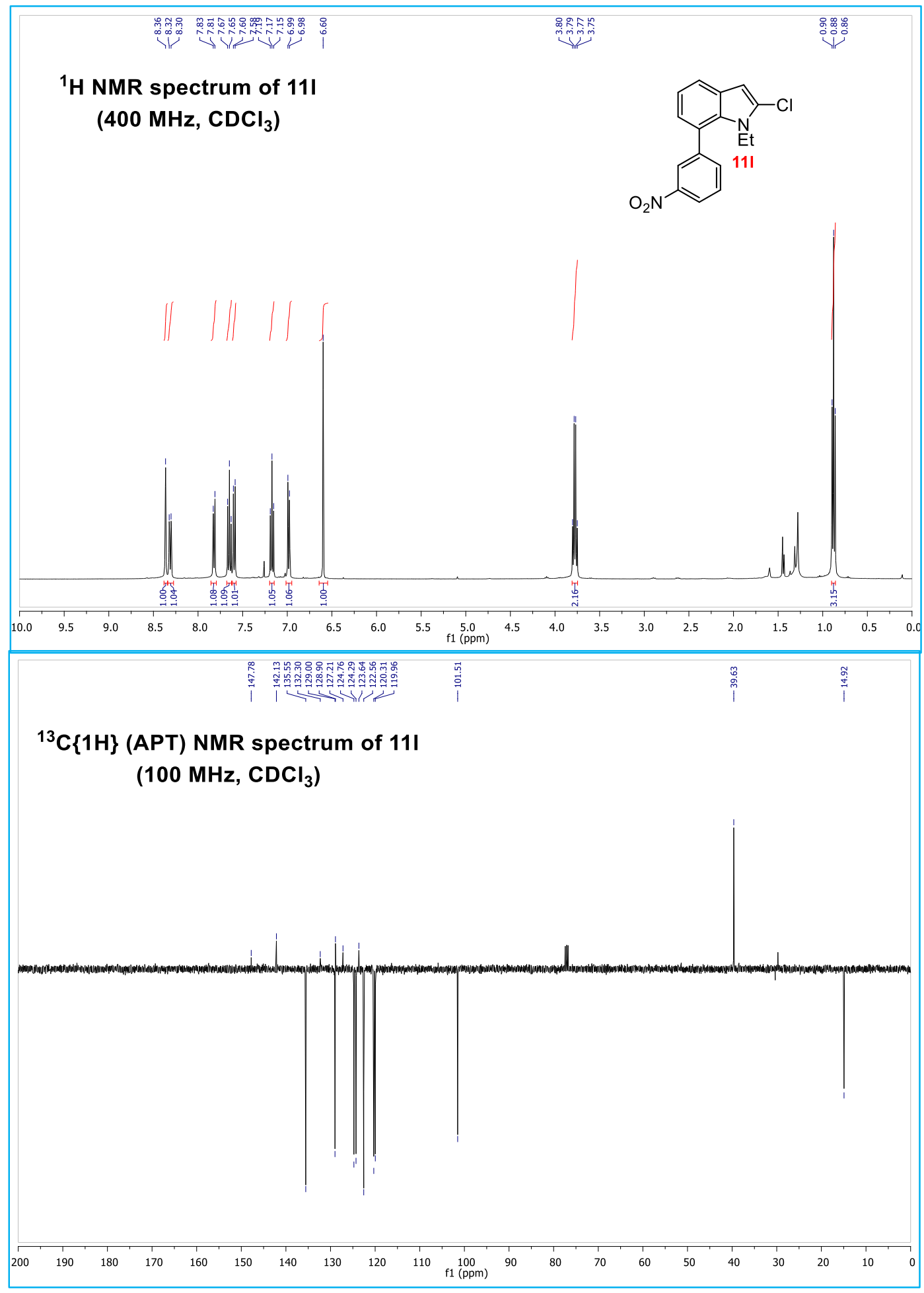


5,10,15-Triethyl-1,6,11-tris(3-methoxyphenyl)-10,15-dihydro-5H-diindolo[3,2-a:3',2'c]carbazole (9m)

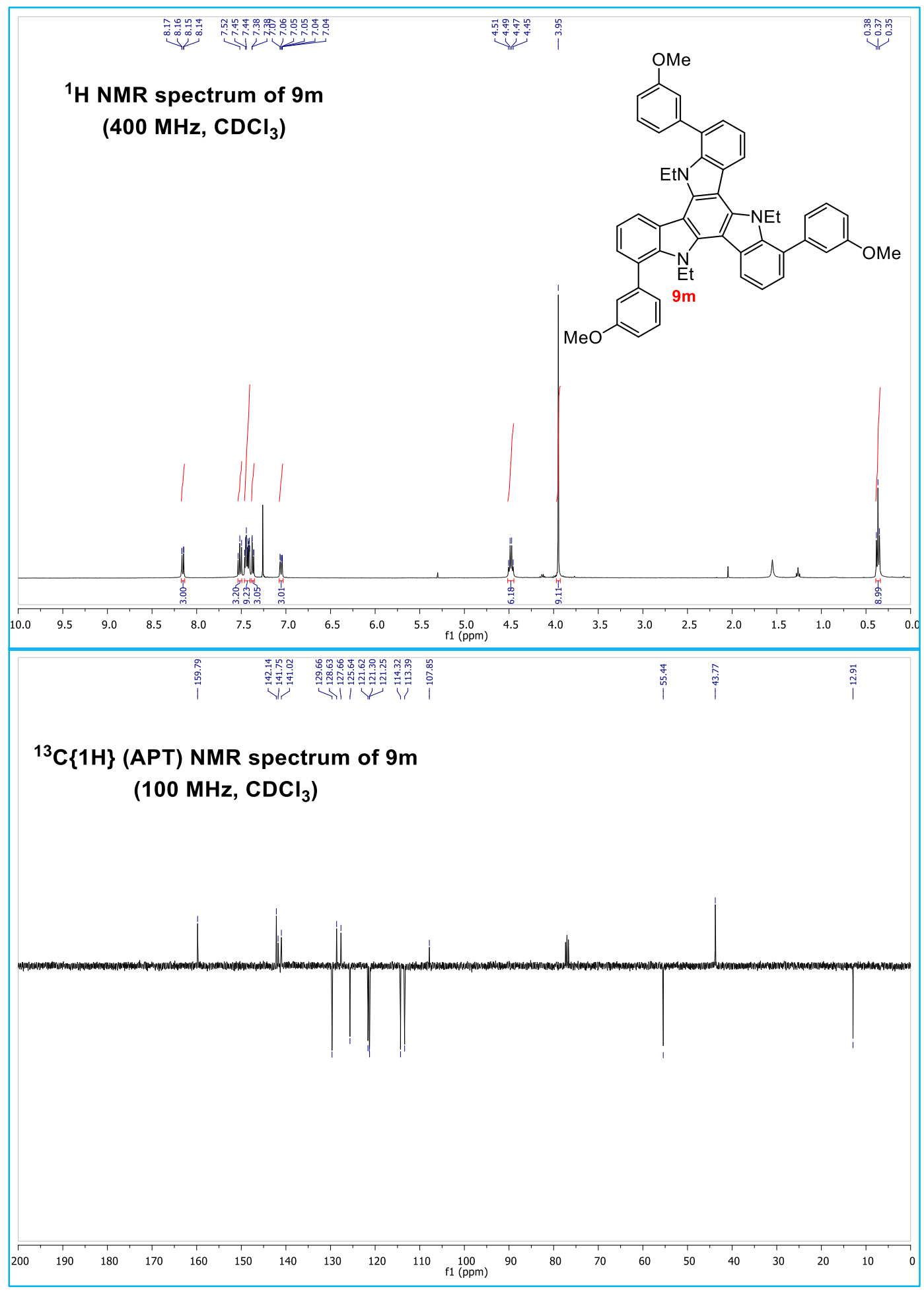


5,10,15-Triethyl-1,6,11-tri-m-tolyl-10,15-dihydro-5H-diindolo[3,2-a:3',2'-c]carbazole (9n)

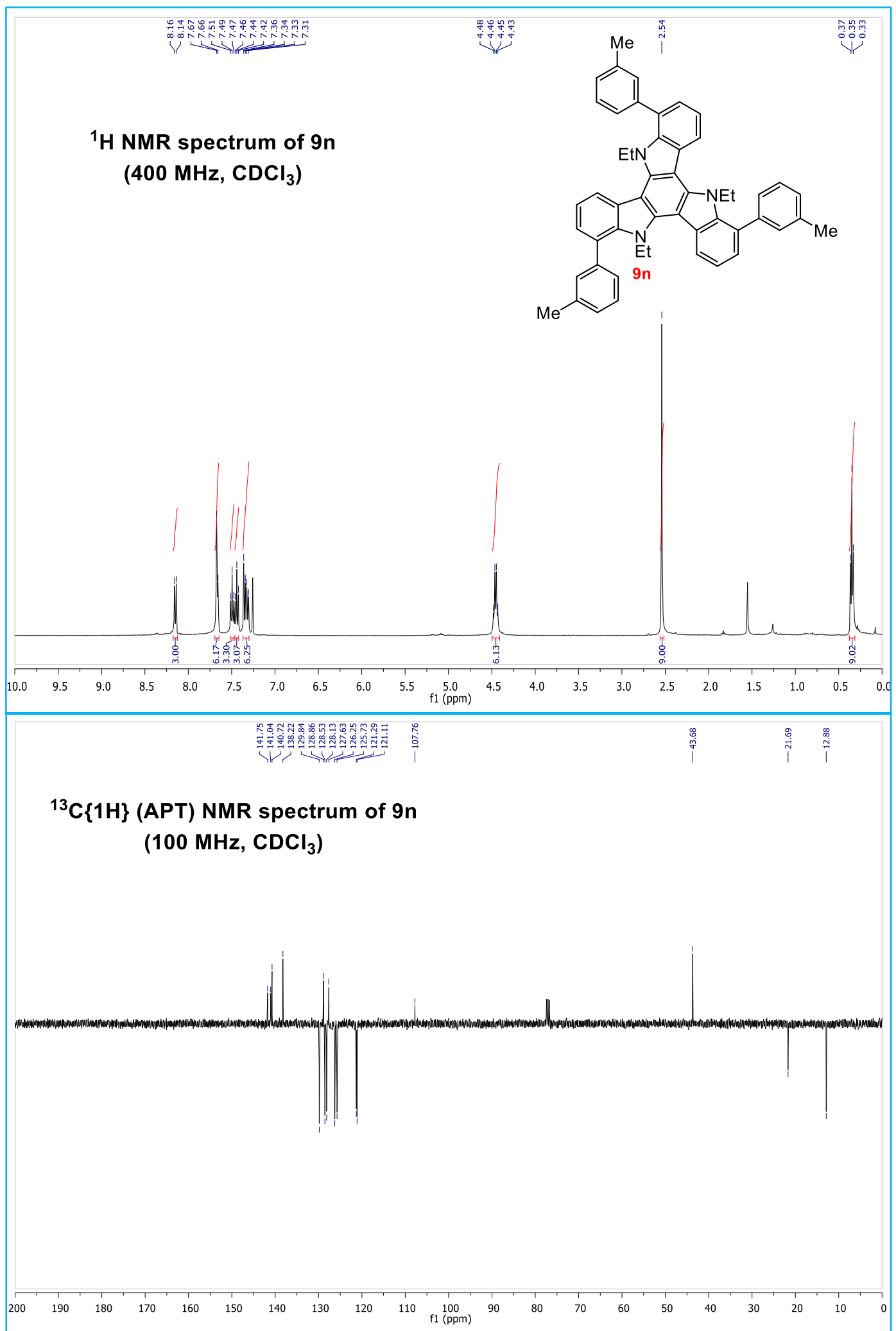


2-Chloro-1-ethyl-7-(pyridin-4-yl)-1H-indole (11o)

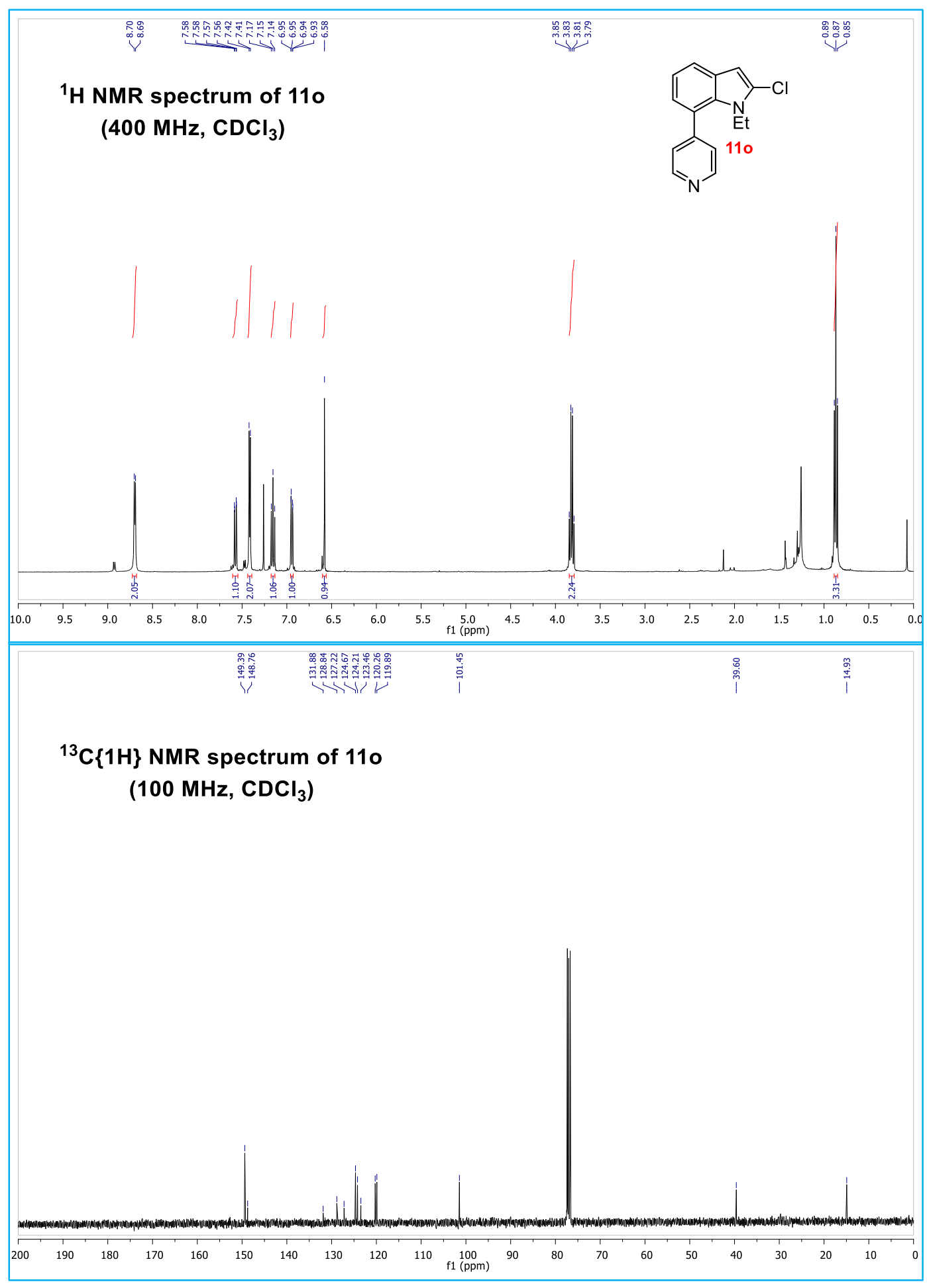


5,10,15-Triethyl-1,6,11-tri(thiophen-2-yl)-10,15-dihydro-5H-diindolo[3,2- $a: 3^{\prime}, 2^{\prime}$ - $c$ ]carbazole (9p)

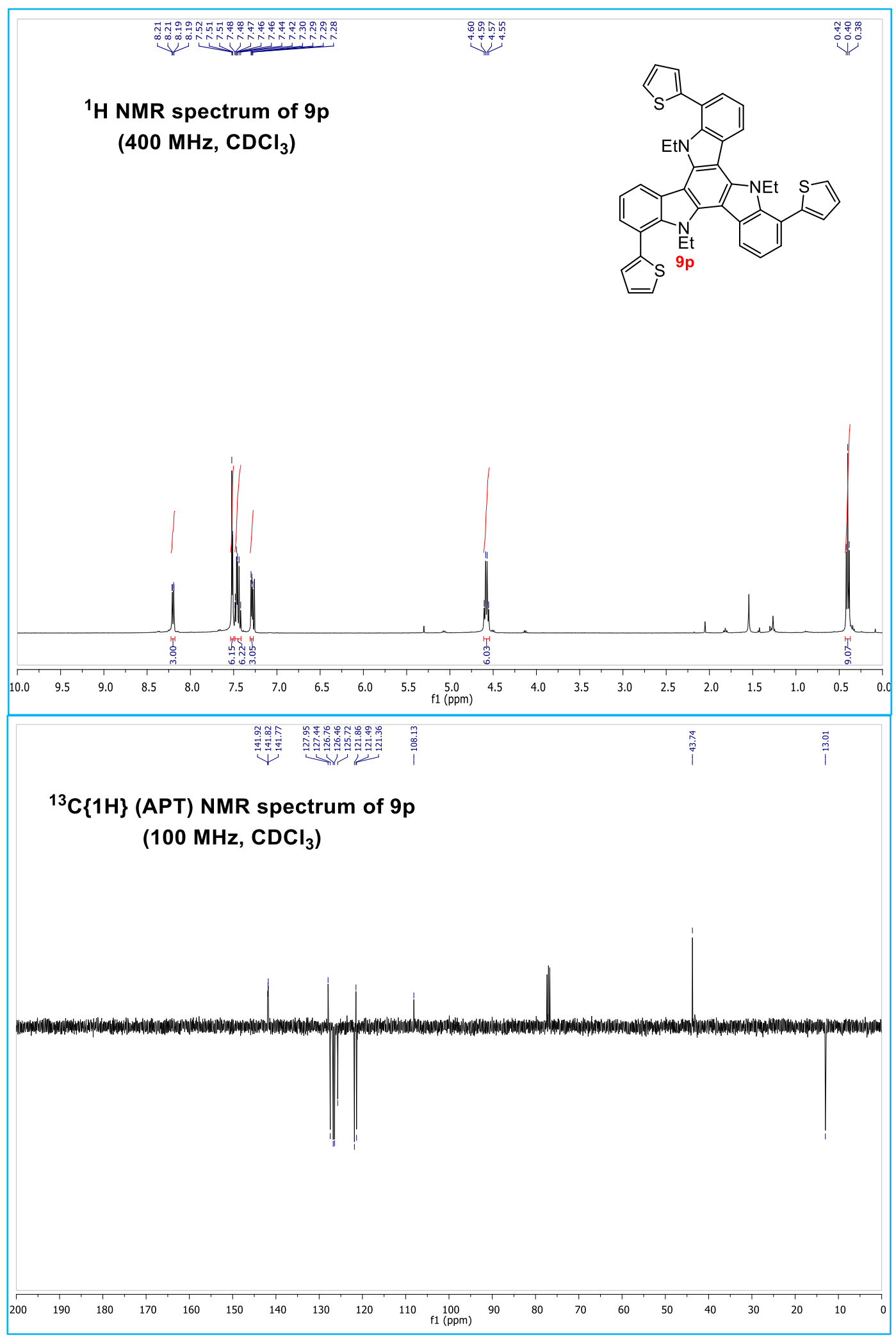


1,6,11-Tribromo-5,10,15-triethyl-10,15-dihydro-5H-diindolo[3,2-a:3',2'-c]carbazole (10)

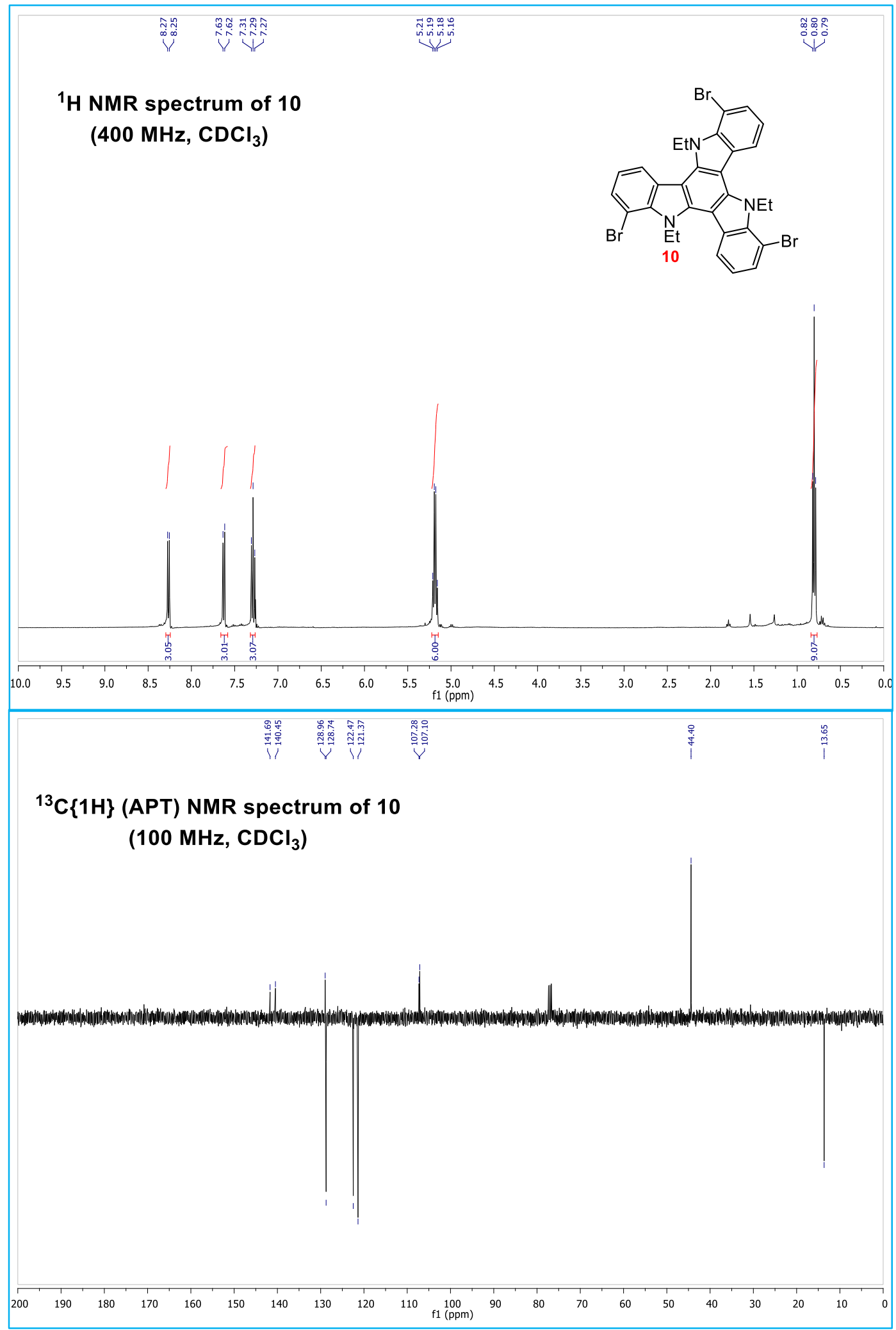


4,4',4'-(5,10,15-triethyl-10,15-dihydro-5H-diindolo[3,2-a:3',2'-c]carbazole-1,6,11triyl)tribenzonitrile (9f)

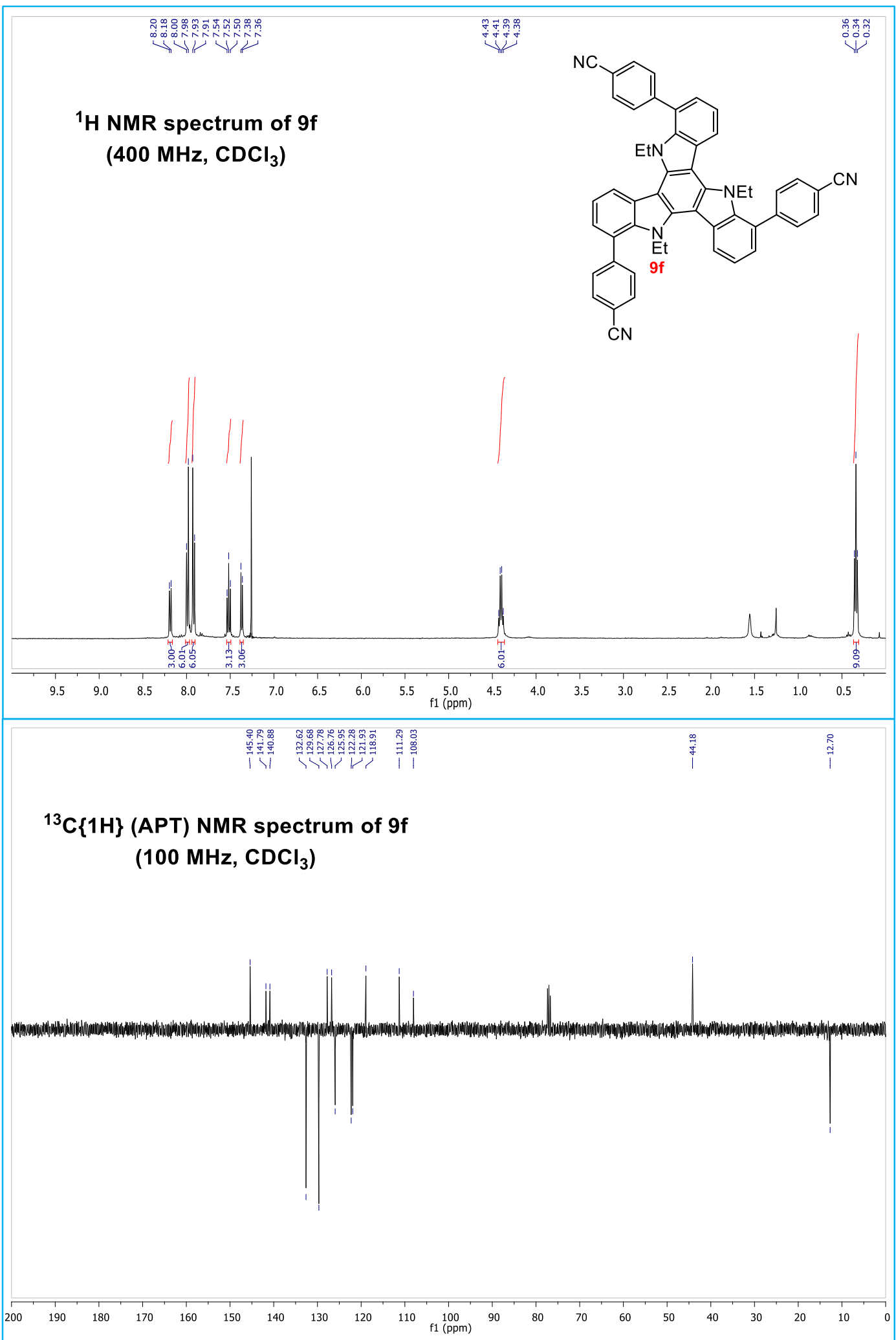


5,10,15-triethyl-1,6,11-tris(4-(trifluoromethyl)phenyl)-10,15-dihydro-5H-diindolo[3,2$a: 3$ ',2'-c] carbazole $(9 \mathrm{~g})$

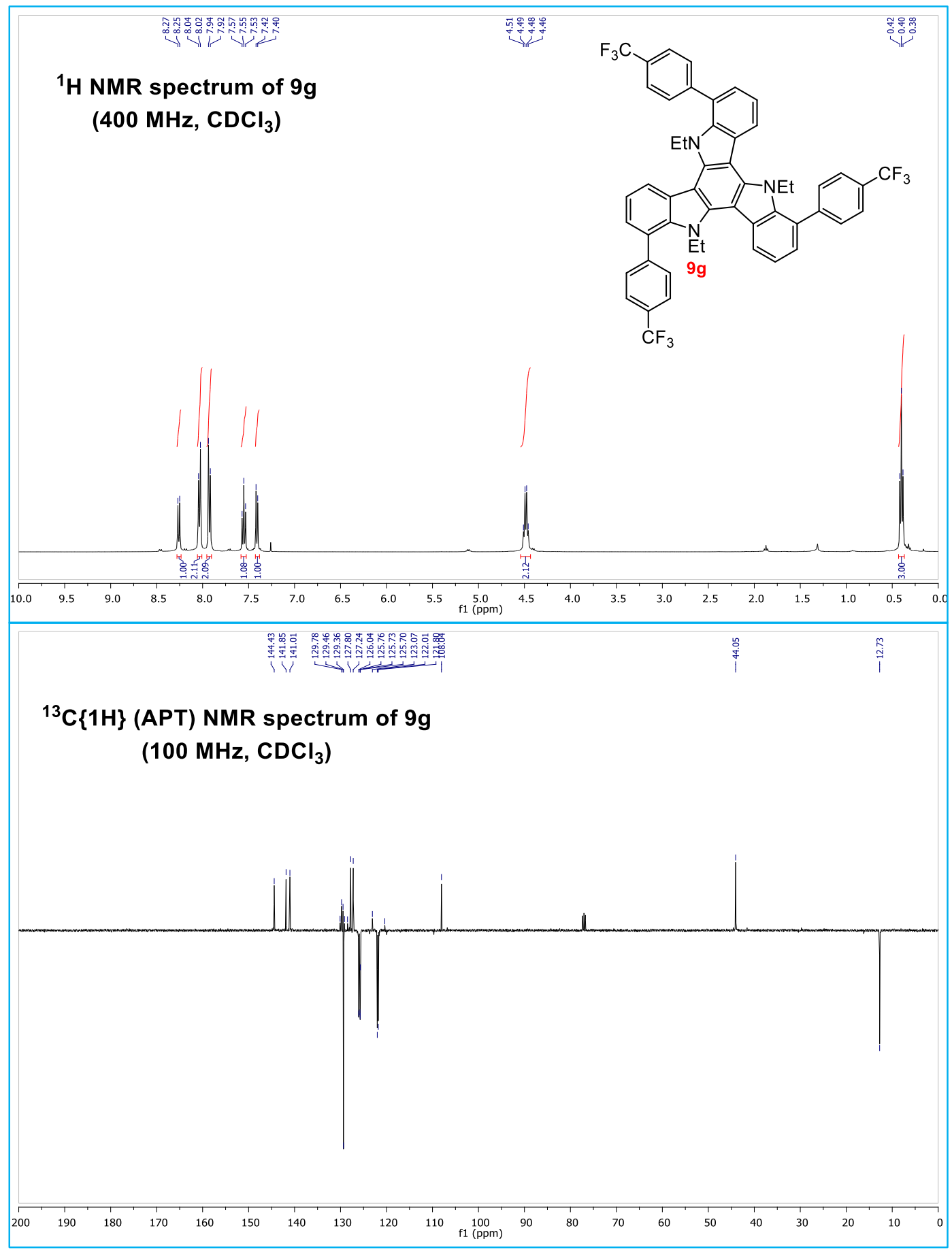


5,10,15-triethyl-1,6,11-tris(4-fluorophenyl)-10,15-dihydro-5H-diindolo[3,2-a:3',2'c]carbazole (9h)
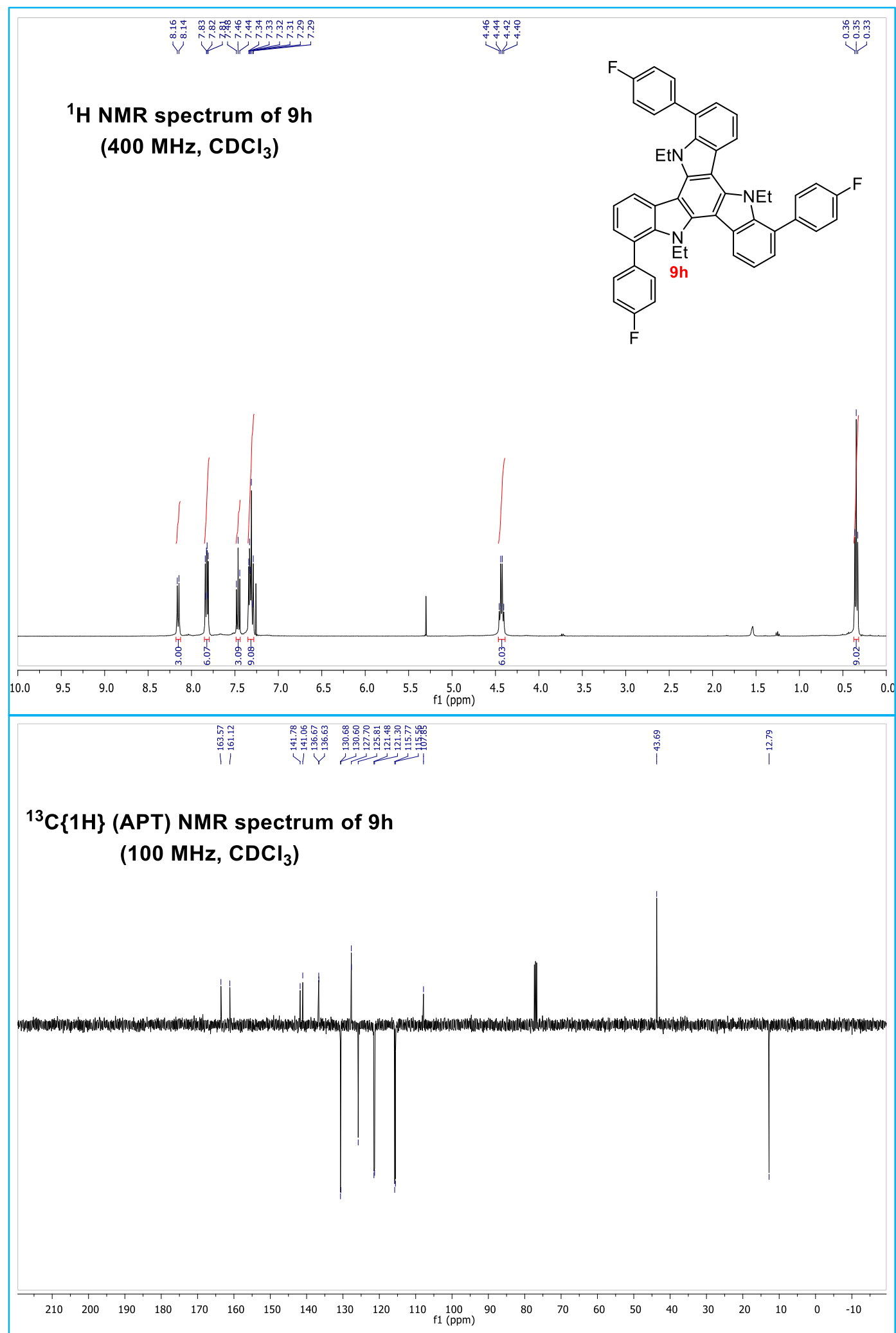
5,10,15-triethyl-1,6,11-tris(3-nitrophenyl)-10,15-dihydro-5H-diindolo[3,2-a:3',2'$c$ carbazole (9I)

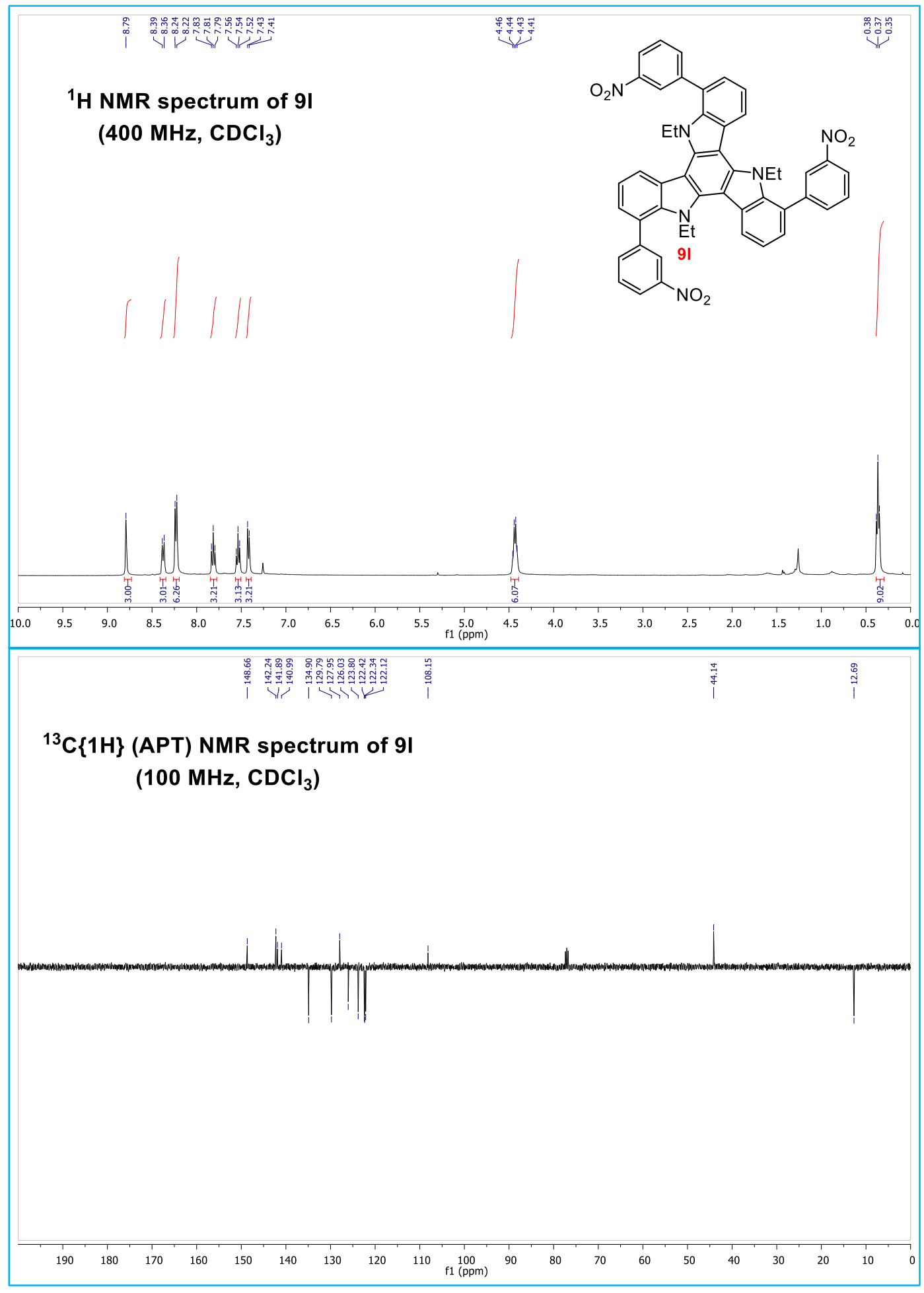


5,10,15-triethyl-1,6,11-tri(pyridin-4-yl)-10,15-dihydro-5H-diindolo[3,2-a:3',2'-c]carbazole (90)

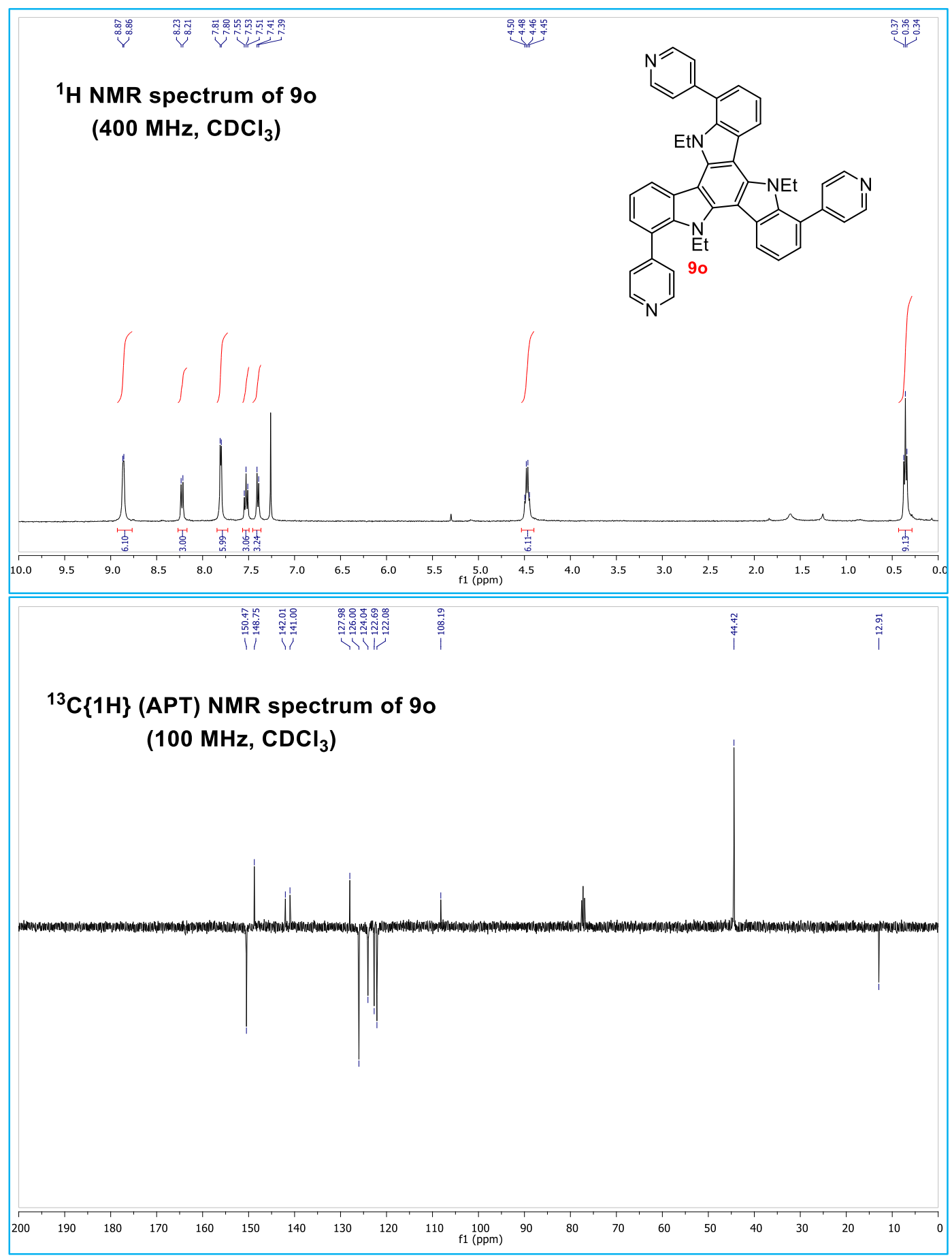




\section{Cartesian coordinates and total energy of optimized structure}

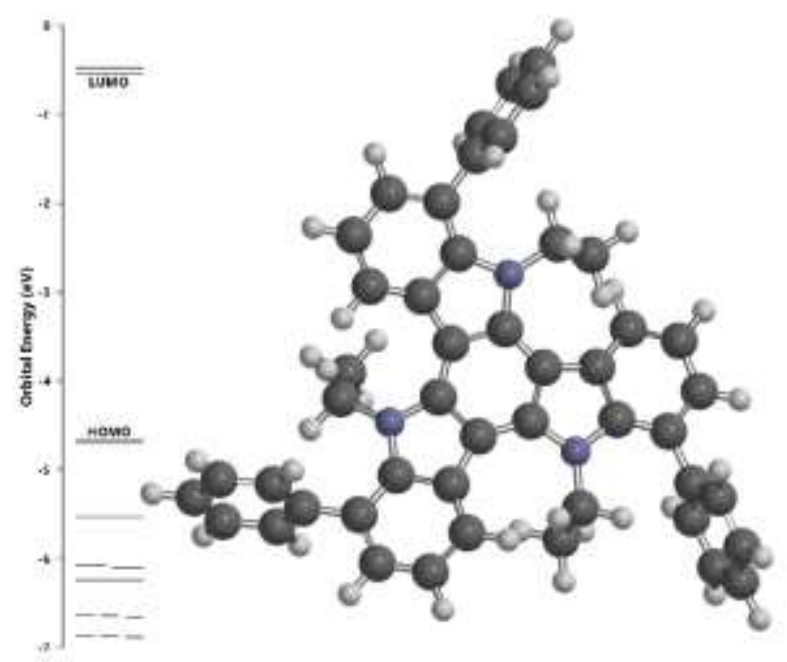

Figure S1. Optimized geometry by semi-empirical calculation using the PM6 of 9a.

Table S1. Atom coordinates and total energy of the optimized geometry of 9a.

Total Energy: $754.1472 \mathrm{~kJ} / \mathrm{mol}$. Zero imaginary frequency.

Cartesian Coordinates (Angstroms)

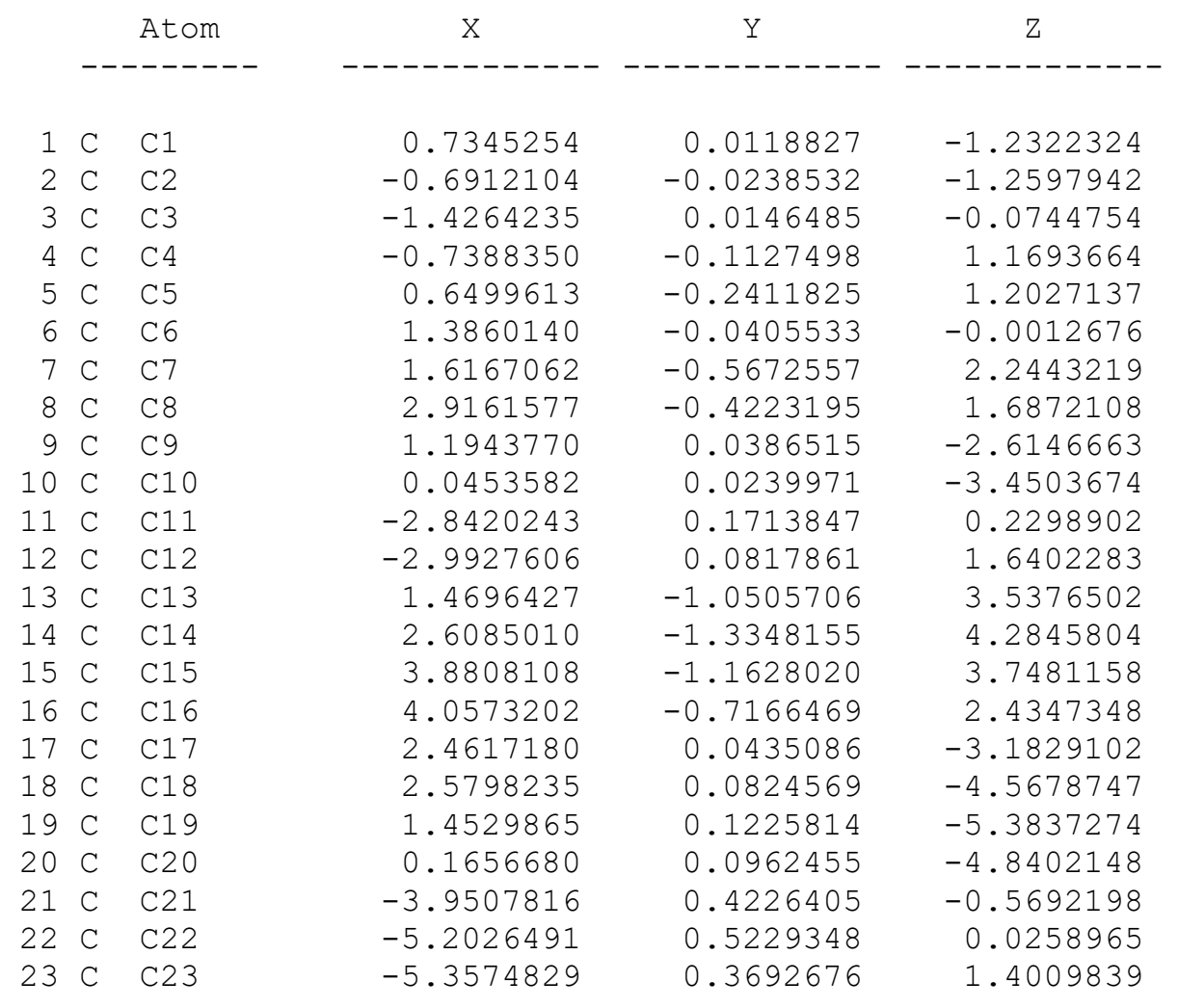




\begin{tabular}{|c|c|c|c|c|c|}
\hline 24 & $\mathrm{C}$ & C24 & -4.2575576 & 0.1441931 & 2.2323442 \\
\hline 25 & $\mathrm{C}$ & $\mathrm{C} 25$ & -0.9832966 & 0.1569828 & -5.7567157 \\
\hline 26 & $\mathrm{C}$ & $\mathrm{C} 26$ & 5.4256481 & -0.6008689 & 1.9071815 \\
\hline 27 & $\mathrm{C}$ & $\mathrm{C} 27$ & -4.4952521 & -0.0245626 & 3.6741285 \\
\hline 28 & $\mathrm{C}$ & $\mathrm{C} 28$ & -1.3724763 & -0.9819069 & -6.4658493 \\
\hline 29 & $\mathrm{C}$ & $\mathrm{C} 29$ & -2.4358684 & -0.9124481 & -7.3587221 \\
\hline 30 & $\mathrm{C}$ & $\mathrm{C} 30$ & -3.1113848 & 0.2888759 & -7.5484830 \\
\hline 31 & $\mathrm{C}$ & C31 & -2.7247824 & 1.4232044 & -6.8425581 \\
\hline 32 & $\mathrm{C}$ & C32 & -1.6627501 & 1.3618695 & -5.9466419 \\
\hline 33 & $\mathrm{C}$ & C33 & 6.1827310 & 0.5416148 & 2.1768182 \\
\hline 34 & $\mathrm{C}$ & C34 & 7.4877015 & 0.6346660 & 1.7063318 \\
\hline 35 & $\mathrm{C}$ & C35 & 8.0399621 & -0.4071709 & 0.9681268 \\
\hline 36 & $\mathrm{C}$ & C36 & 7.2859319 & -1.5442247 & 0.6979071 \\
\hline 37 & $\mathrm{C}$ & C37 & 5.9798821 & -1.6454338 & 1.1652360 \\
\hline 38 & $\mathrm{C}$ & C38 & -4.7764337 & 1.0877993 & 4.4711709 \\
\hline 39 & $\mathrm{C}$ & C39 & -5.0360437 & 0.9211260 & 5.8267821 \\
\hline 40 & $\mathrm{C}$ & $\mathrm{C} 40$ & -5.0188862 & -0.3508307 & 6.3900935 \\
\hline 41 & $\mathrm{C}$ & C41 & -4.7400603 & -1.4590474 & 5.5971065 \\
\hline 42 & $\mathrm{C}$ & $\mathrm{C} 42$ & -4.4778609 & -1.3008324 & 4.2405040 \\
\hline 43 & $\mathrm{~N}$ & N43 & 2.8106809 & -0.0673580 & 0.2834752 \\
\hline 44 & $\mathrm{C}$ & C44 & 3.3468936 & 2.3975017 & 0.4674848 \\
\hline 45 & $\mathrm{C}$ & C45 & 3.6451202 & 1.0632045 & -0.1901579 \\
\hline 46 & $\mathrm{~N}$ & N4 6 & -1.1539307 & -0.0165840 & -2.6337297 \\
\hline 47 & $\mathrm{C}$ & C4 7 & -1.7954338 & -2.4390728 & -2.9745394 \\
\hline 48 & $\mathrm{C}$ & $\mathrm{C} 48$ & -2.2056739 & -0.9801522 & -3.0408362 \\
\hline 49 & $\mathrm{~N}$ & N49 & -1.6958459 & -0.1211310 & 2.2548479 \\
\hline 50 & $\mathrm{C}$ & C50 & -1.3079166 & 2.1400241 & 3.3143623 \\
\hline 51 & $\mathrm{C}$ & C51 & -1.4001828 & 0.6364839 & 3.4934805 \\
\hline 52 & $\mathrm{H}$ & H52 & 0.4727064 & -1.2144635 & 3.9672110 \\
\hline 53 & $\mathrm{H}$ & H53 & 2.5021528 & -1.7079590 & 5.3085876 \\
\hline 54 & $\mathrm{H}$ & H5 4 & 4.7623864 & -1.3939060 & 4.3565112 \\
\hline 55 & $\mathrm{H}$ & H5 5 & 3.3624253 & 0.0106450 & -2.5569343 \\
\hline 56 & $\mathrm{H}$ & H5 6 & 3.5749541 & 0.0862734 & -5.0247649 \\
\hline 57 & $\mathrm{H}$ & H5 7 & 1.5723554 & 0.1723212 & -6.4718961 \\
\hline 58 & $\mathrm{H}$ & H5 8 & -3.8463944 & 0.5467311 & -1.6548061 \\
\hline 59 & $\mathrm{H}$ & H59 & -6.0822680 & 0.7201749 & -0.5958042 \\
\hline 60 & $\mathrm{H}$ & $\mathrm{H} 60$ & -6.3578159 & 0.4303839 & 1.8440810 \\
\hline 61 & $\mathrm{H}$ & $\mathrm{H} 61$ & -0.8386059 & -1.9264724 & -6.3116755 \\
\hline 62 & $\mathrm{H}$ & H62 & -2.7402608 & -1.8056193 & -7.9139990 \\
\hline 63 & $\mathrm{H}$ & $\mathrm{H} 63$ & -3.9467848 & 0.3413633 & -8.2540543 \\
\hline 64 & $\mathrm{H}$ & H 64 & -3.2562057 & 2.3686402 & -6.9925228 \\
\hline 65 & $\mathrm{H}$ & $\mathrm{H} 65$ & -1.3565437 & 2.2545672 & -5.3904675 \\
\hline 66 & $\mathrm{H}$ & H 66 & 5.7425783 & 1.3611896 & 2.7560949 \\
\hline 67 & $\mathrm{H}$ & H 67 & 8.0812348 & 1.5297175 & 1.9192668 \\
\hline 68 & $\mathrm{H}$ & H68 & 9.0684426 & -0.3319052 & 0.6007102 \\
\hline 69 & $\mathrm{H}$ & H69 & 7.7209400 & -2.3639893 & 0.1171322 \\
\hline 70 & $\mathrm{H}$ & $\mathrm{H} 7 \mathrm{O}$ & 5.3848418 & -2.5407001 & 0.9541214 \\
\hline 71 & $\mathrm{H}$ & H71 & -4.7876380 & 2.0879486 & 4.0233204 \\
\hline 72 & $\mathrm{H}$ & $\mathrm{H} 72$ & -5.2556186 & 1.7936014 & 6.4507979 \\
\hline 73 & $\mathrm{H}$ & H73 & -5.2256925 & -0.4794355 & 7.4574817 \\
\hline 74 & $\mathrm{H}$ & H7 4 & -4.7277168 & -2.4599815 & 6.0405332 \\
\hline 75 & $\mathrm{H}$ & H7 5 & -4.2588538 & -2.1728077 & 3.6144245 \\
\hline 76 & $\mathrm{H}$ & H7 6 & 3.9692233 & 3.1869293 & 0.0265433 \\
\hline 77 & $\mathrm{H}$ & H77 & 2.2978329 & 2.6975889 & 0.3422760 \\
\hline 78 & $\mathrm{H}$ & H78 & 3.5592192 & 2.3779598 & 1.5459730 \\
\hline 79 & $\mathrm{H}$ & H79 & 3.5158803 & 1.1395983 & -1.2903983 \\
\hline 80 & $\mathrm{H}$ & $\mathrm{H} 80$ & 4.7024941 & 0.7738963 & -0.0118502 \\
\hline
\end{tabular}




$\begin{array}{llllrr}81 & \mathrm{H} & \mathrm{H} 81 & -1.4746375 & -2.7351072 & -1.9664569 \\ 82 & \mathrm{H} & \mathrm{H} 82 & -2.6389677 & -3.0838555 & -3.2530119 \\ 83 & \mathrm{H} & \mathrm{H} 83 & -0.9699438 & -2.6642470 & -3.6646737 \\ 84 & \mathrm{H} & \mathrm{H} 84 & -2.5042865 & -0.7100557 & -4.0757369 \\ 85 & \mathrm{H} & \mathrm{H} 85 & -3.0939826 & -0.8011569 & -2.3993283 \\ 86 & \mathrm{H} & \mathrm{H} 86 & -1.0351783 & 2.6197872 & 4.2631793 \\ 87 & \mathrm{H} & \mathrm{H} 87 & -0.5490873 & 2.4239112 & 2.5725378 \\ 88 & \mathrm{H} & \mathrm{H} 88 & -2.2653416 & 2.5731451 & 2.9920144 \\ 89 & \mathrm{H} & \mathrm{H} 89 & -0.4522189 & 0.2366373 & 3.9119388 \\ 90 & \mathrm{H} & \mathrm{H} 90 & -2.1959340 & 0.3834928 & 4.2255524\end{array}$

Table S2. Atomic charges of 9a calculated by B3LYP/6-31G (d,p) method

\begin{tabular}{|l|l|l|l|}
\hline Atom Label & Natural charge & Mulliken charge & Electrostatic charge \\
\hline C1 & -0.082 & -0.082 & -0.486 \\
\hline C2 & -0.024 & -0.024 & +0.497 \\
\hline C4 & -0.086 & -0.086 & -0.504 \\
\hline C5 & -0.027 & -0.027 & +0.508 \\
\hline C6 & -0.090 & -0.090 & -0.495 \\
\hline C7 & -0.043 & -0.025 & +0.507 \\
\hline C8 & -0.086 & -0.043 & +0.217 \\
\hline C9 & -0.042 & -0.086 & +0.153 \\
\hline C10 & -0.086 & -0.042 & +0.232 \\
\hline C11 & -0.044 & -0.086 & -0.080 \\
\hline C12 & -0.087 & -0.044 & +0.116 \\
\hline C13 & -0.079 & -0.087 & +0.209 \\
\hline C14 & -0.116 & -0.079 & -0.168 \\
\hline C15 & -0.085 & -0.085 & -0.162 \\
\hline C16 & -0.029 & -0.078 & -0.029 \\
\hline
\end{tabular}




\begin{tabular}{|c|c|c|c|}
\hline C18 & -0.118 & -0.118 & -0.094 \\
\hline C19 & -0.085 & -0.085 & -0.171 \\
\hline C20 & -0.031 & -0.031 & -0.132 \\
\hline C21 & -0.077 & -0.077 & -0.221 \\
\hline $\mathrm{C} 22$ & -0.119 & -0.119 & -0.108 \\
\hline $\mathrm{C} 23$ & -0.084 & -0.084 & -0.140 \\
\hline C24 & -0.033 & -0.033 & -0.199 \\
\hline $\mathrm{C} 25$ & -0.033 & -0.033 & +0.362 \\
\hline $\mathrm{C} 26$ & -0.033 & -0.033 & +0.414 \\
\hline $\mathrm{C} 27$ & -0.033 & -0.033 & +0.407 \\
\hline $\mathrm{C} 28$ & -0.097 & -0.097 & -0.234 \\
\hline C29 & -0.104 & -0.104 & -0.109 \\
\hline C30 & -0.102 & -0.102 & -0.134 \\
\hline C31 & -0.104 & -0.104 & -0.055 \\
\hline C32 & -0.086 & -0.086 & -0.313 \\
\hline C33 & -0.098 & -0.098 & -0.271 \\
\hline C34 & -0.105 & -0.105 & -0.093 \\
\hline C35 & -0.102 & -0.102 & -0.147 \\
\hline C36 & -0.103 & -0.103 & -0.049 \\
\hline C37 & -0.085 & -0.085 & -0.327 \\
\hline C38 & -0.097 & -0.097 & -0.248 \\
\hline C39 & -0.104 & -0.104 & -0.106 \\
\hline C40 & -0.102 & -0.102 & -0.140 \\
\hline C41 & -0.103 & -0.103 & -0.048 \\
\hline
\end{tabular}




\begin{tabular}{|c|c|c|c|}
\hline $\mathrm{C} 42$ & -0.087 & -0.087 & -0.339 \\
\hline C44 & -0.139 & -0.139 & -0.656 \\
\hline C45 & -0.110 & -0.110 & +0.119 \\
\hline C47 & -0.139 & -0.139 & -0.641 \\
\hline C48 & -0.115 & -0.115 & +0.166 \\
\hline C50 & -0.137 & -0.137 & -0.640 \\
\hline C51 & -0.119 & -0.119 & +0.206 \\
\hline H52 & +0.119 & +0.119 & +0.151 \\
\hline H53 & +0.104 & +0.104 & +0.105 \\
\hline H54 & +0.109 & +0.109 & +0.123 \\
\hline H55 & +0.120 & +0.120 & +0.141 \\
\hline H56 & +0.104 & +0.104 & +0.109 \\
\hline H57 & +0.109 & +0.109 & +0.123 \\
\hline H58 & +0.120 & +0.120 & +0.144 \\
\hline H59 & +0.104 & +0.104 & +0.109 \\
\hline H60 & +0.109 & +0.109 & +0.119 \\
\hline H61 & +0.111 & +0.111 & +0.126 \\
\hline H62 & +0.104 & +0.104 & +0.115 \\
\hline H63 & +0.103 & +0.103 & +0.111 \\
\hline H64 & +0.104 & +0.104 & +0.105 \\
\hline H65 & +0.114 & +0.114 & +0.158 \\
\hline H66 & +0.110 & +0.110 & +0.133 \\
\hline H67 & +0.104 & +0.104 & +0.112 \\
\hline H68 & +0.103 & +0.103 & +0.113 \\
\hline
\end{tabular}




\begin{tabular}{|c|c|c|c|}
\hline H69 & +0.104 & +0.104 & +0.104 \\
\hline H70 & +0.115 & +0.115 & +0.161 \\
\hline H71 & +0.111 & +0.111 & +0.127 \\
\hline H72 & +0.104 & +0.104 & +0.114 \\
\hline H73 & +0.104 & +0.104 & +0.112 \\
\hline H74 & +0.105 & +0.105 & +0.105 \\
\hline H75 & +0.114 & +0.114 & +0.162 \\
\hline H76 & +0.048 & +0.048 & +0.163 \\
\hline H77 & +0.053 & +0.053 & +0.201 \\
\hline H78 & +0.049 & +0.049 & +0.196 \\
\hline H79 & +0.081 & +0.081 & +0.113 \\
\hline H80 & +0.080 & +0.080 & +0.102 \\
\hline H81 & +0.055 & +0.055 & +0.206 \\
\hline H82 & +0.048 & +0.048 & +0.152 \\
\hline H83 & +0.050 & +0.050 & +0.197 \\
\hline H84 & +0.081 & +0.081 & +0.090 \\
\hline H85 & +0.082 & +0.082 & +0.091 \\
\hline H86 & +0.048 & +0.048 & +0.148 \\
\hline H87 & +0.053 & +0.053 & +0.200 \\
\hline H88 & +0.049 & +0.049 & +0.194 \\
\hline H89 & +0.083 & +0.083 & +0.072 \\
\hline H9O & +0.083 & +0.083 & +0.081 \\
\hline $\mathbf{N} 43$ & +0.140 & +0.140 & -0.497 \\
\hline N46 & +0.148 & +0.148 & -0.483 \\
\hline
\end{tabular}



N49 $+0.158$ $+0.158$ $-0.498$

\section{HRMS spectra of compounds}

HRMS spectrum of 4

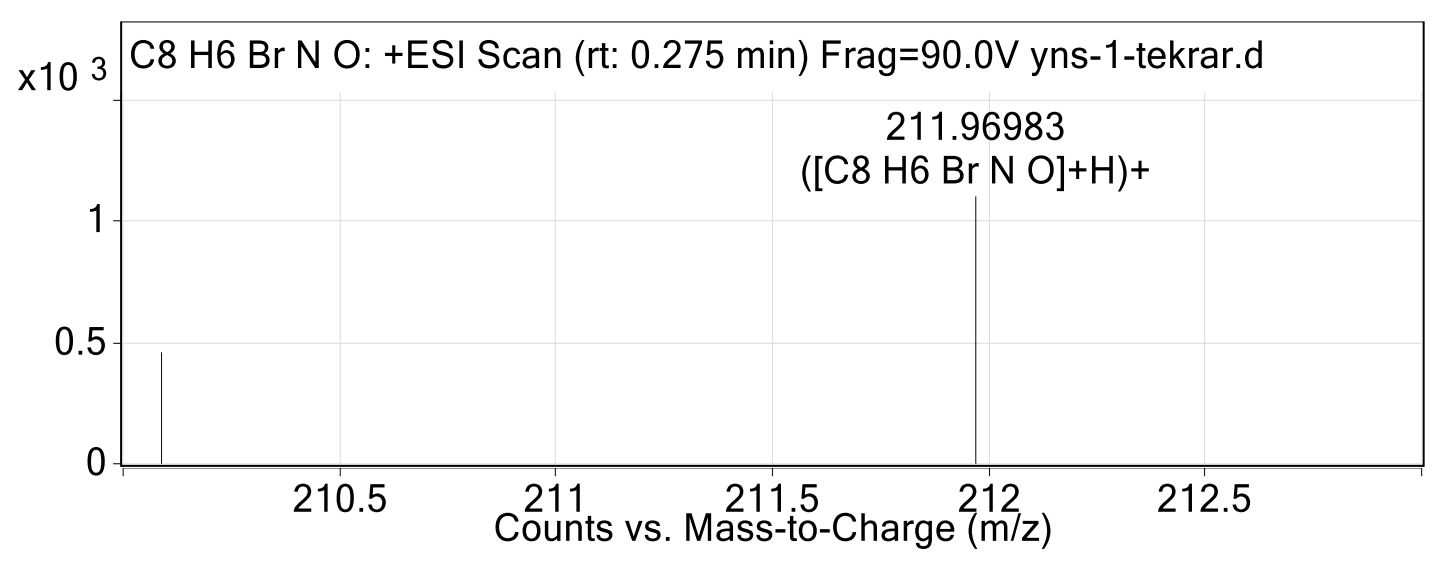

HRMS spectrum of $\mathbf{5}$

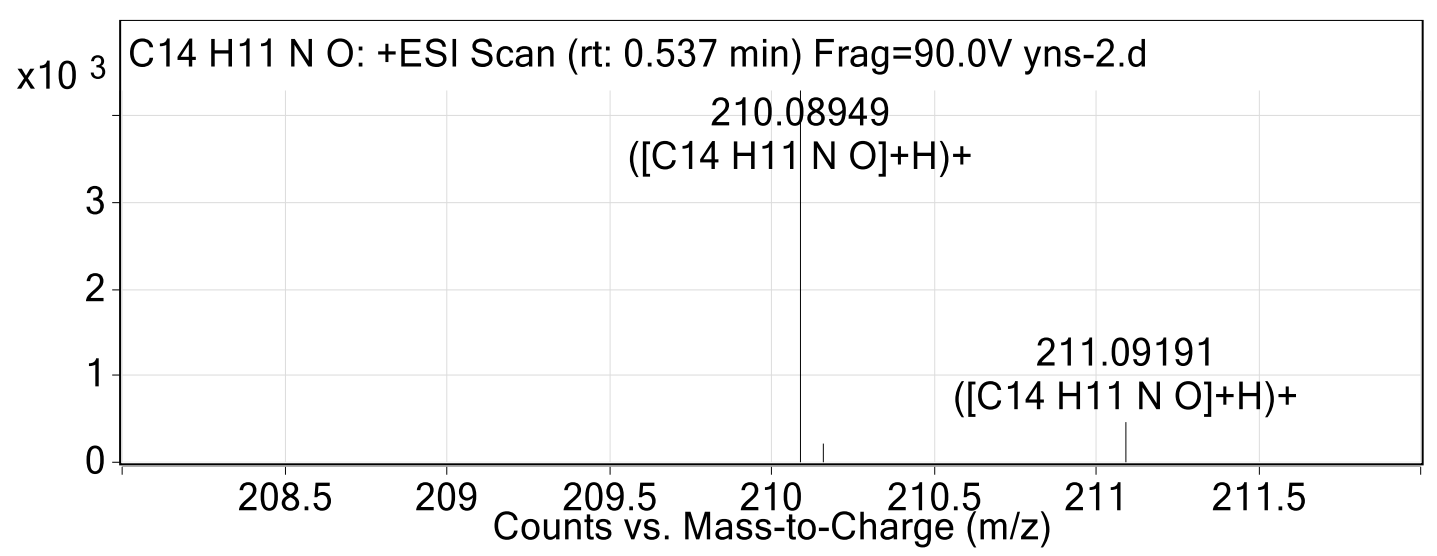

HRMS spectrum of 6

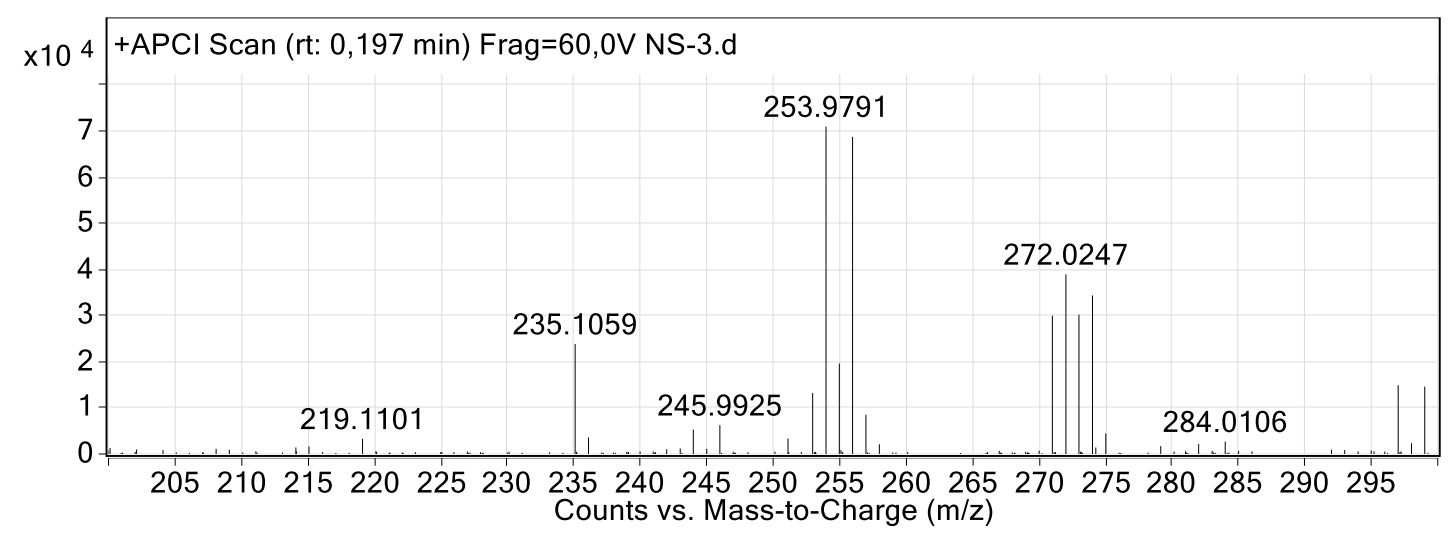


HRMS spectrum of 7

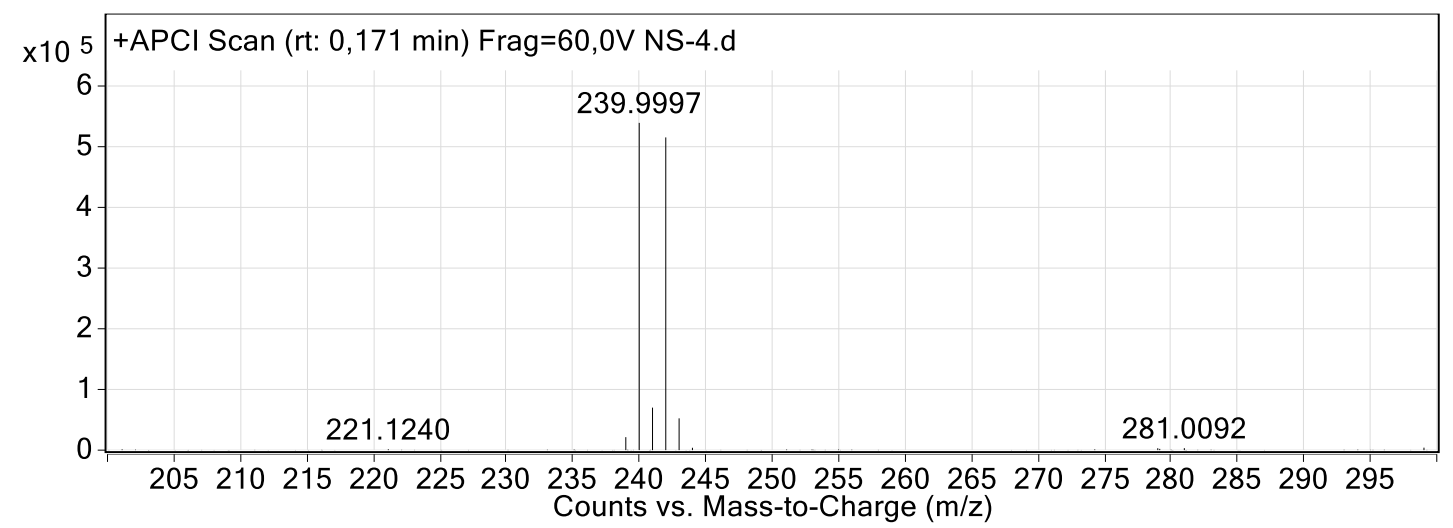

HRMS spectrum of $\mathbf{8 a}$

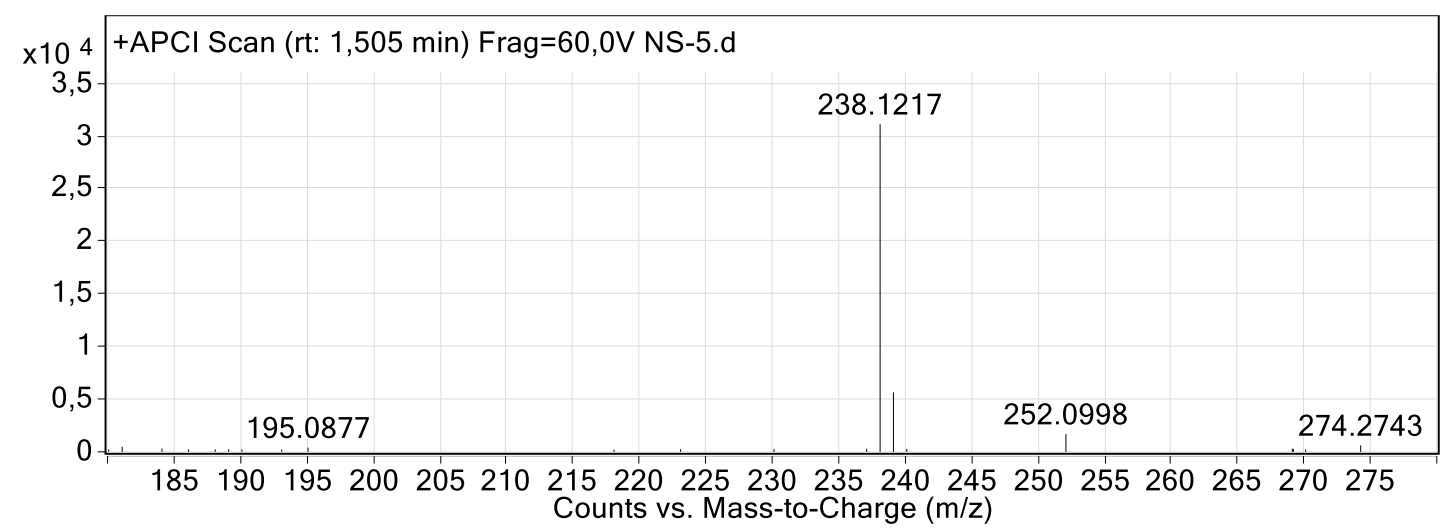

\section{HRMS spectrum of $\mathbf{8 b}$}

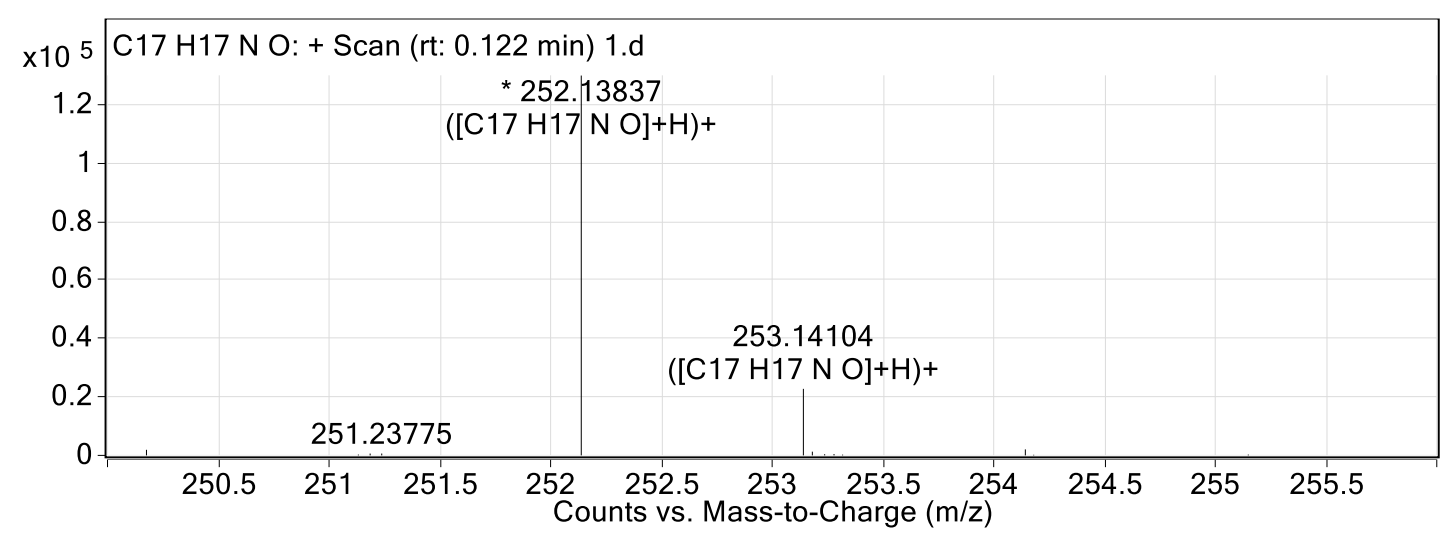


HRMS spectrum of $\mathbf{8 c}$

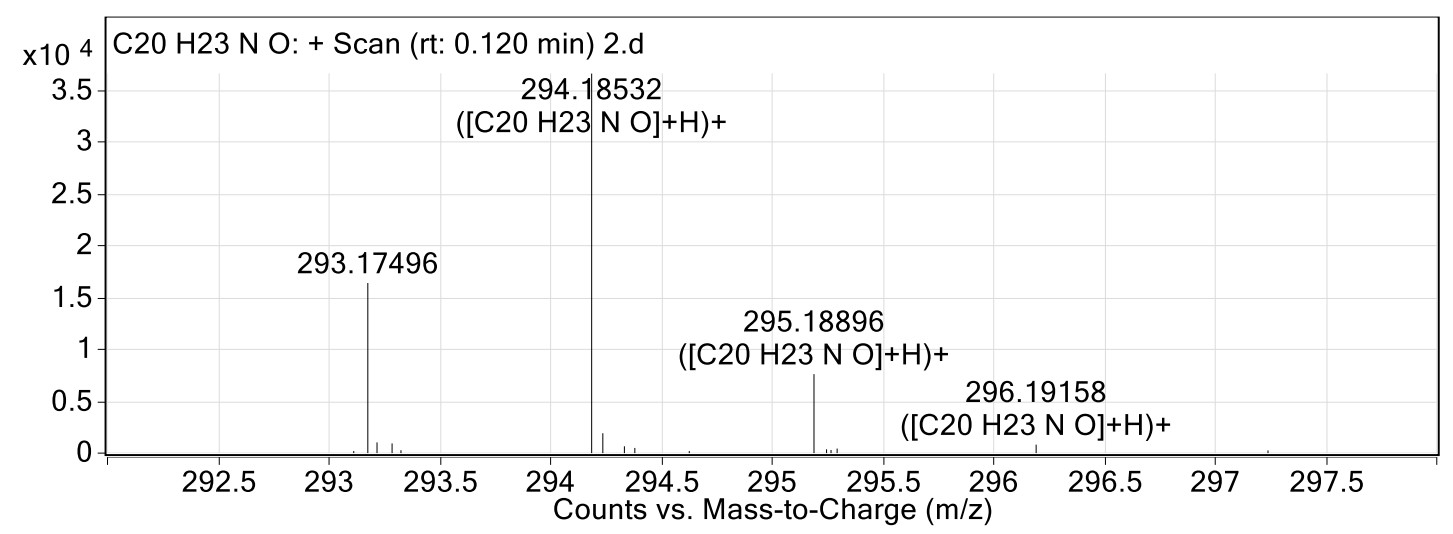

HRMS spectrum of $\mathbf{8 d}$

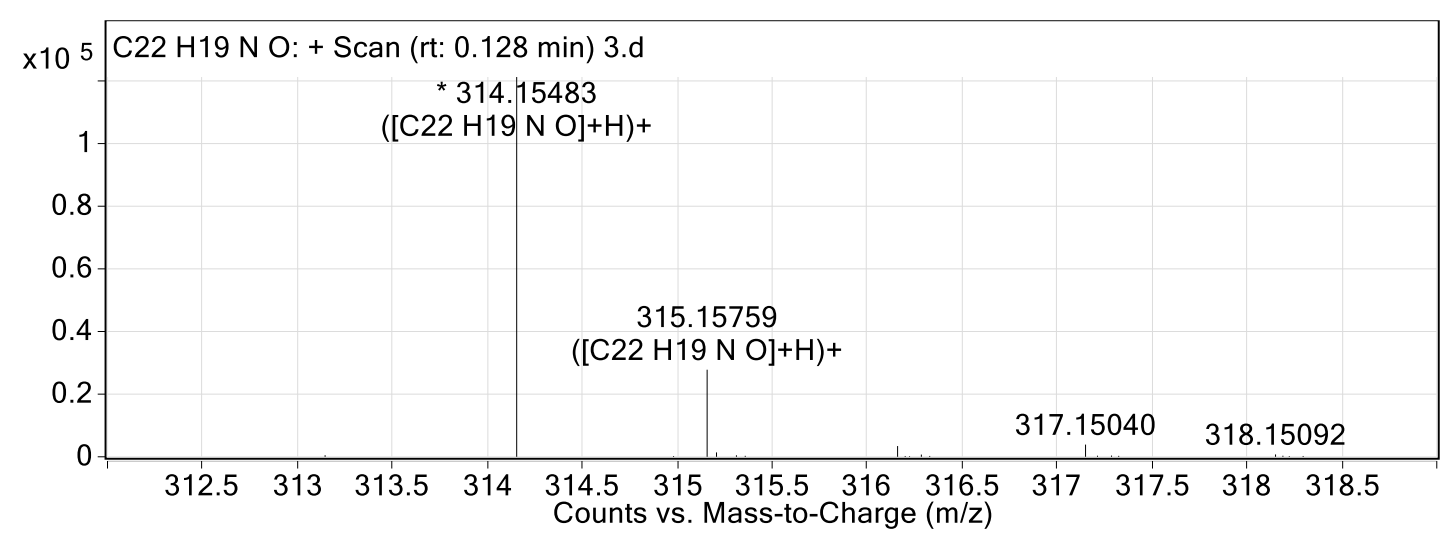

\section{HRMS spectrum of $\mathbf{8 e}$}

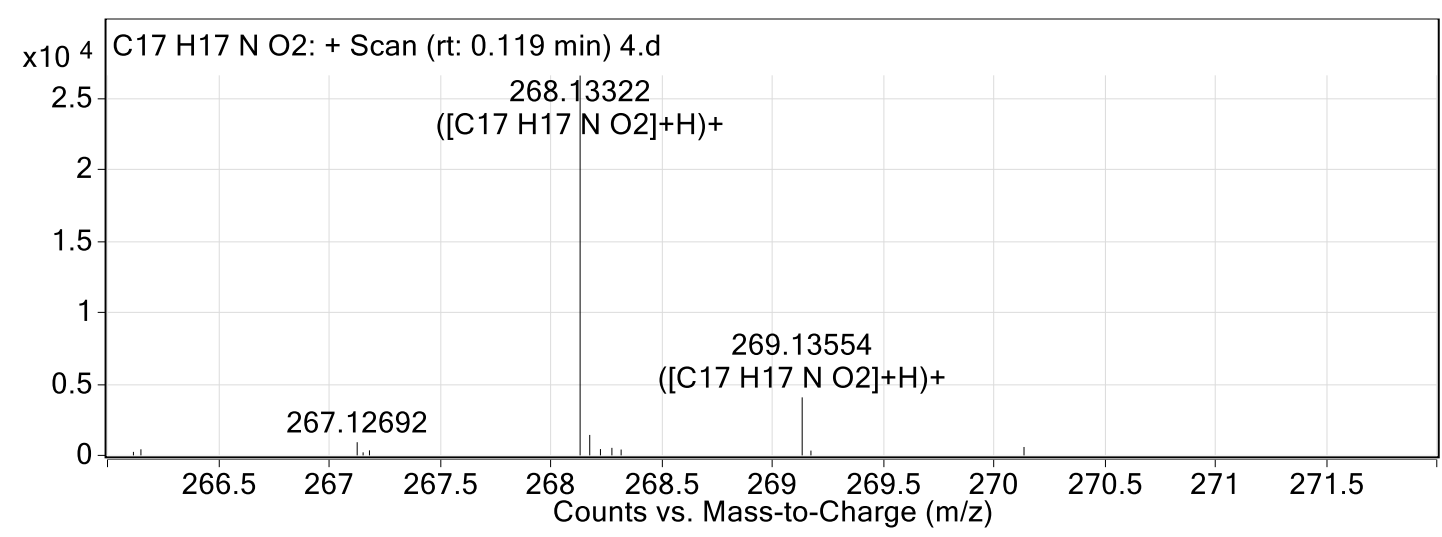


HRMS spectrum of $\mathbf{8 f}$

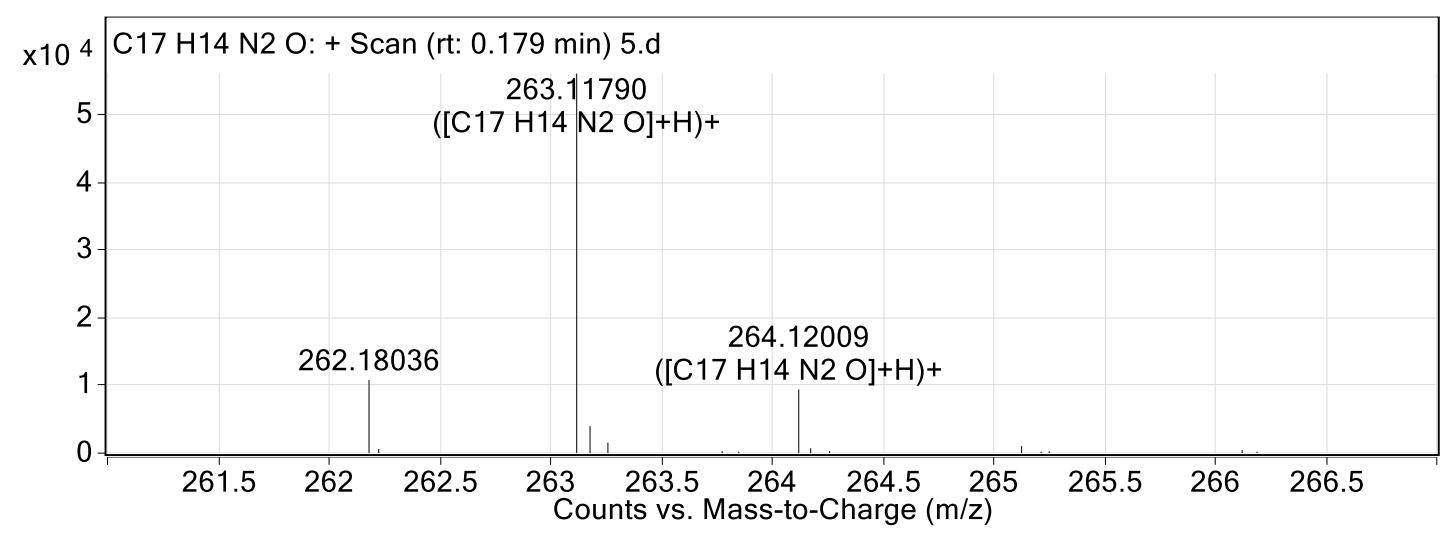

HRMS spectrum of $\mathbf{8 g}$

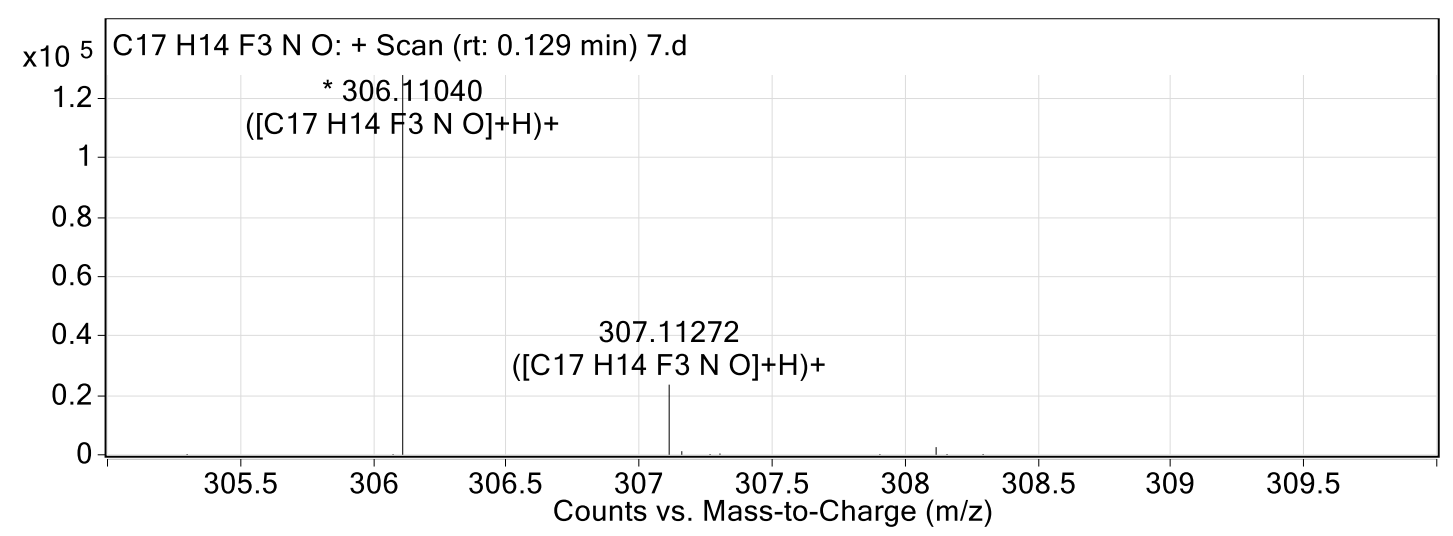

\section{HRMS spectrum of $\mathbf{8 h}$}

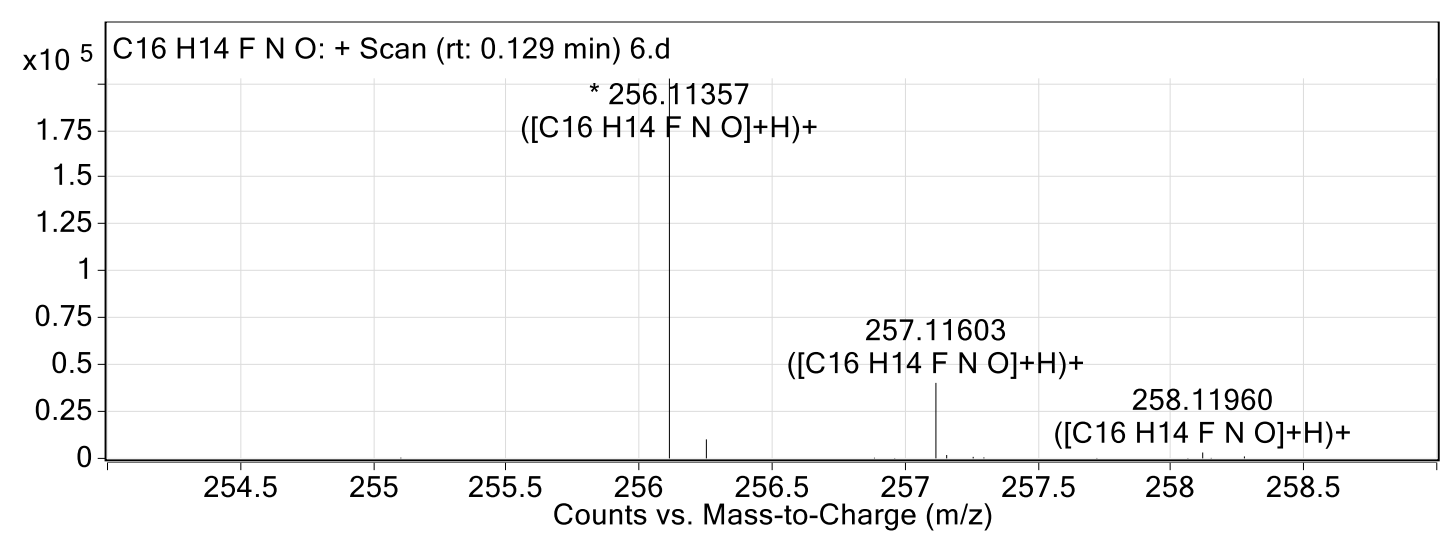


HRMS spectrum of $\mathbf{8 j}$

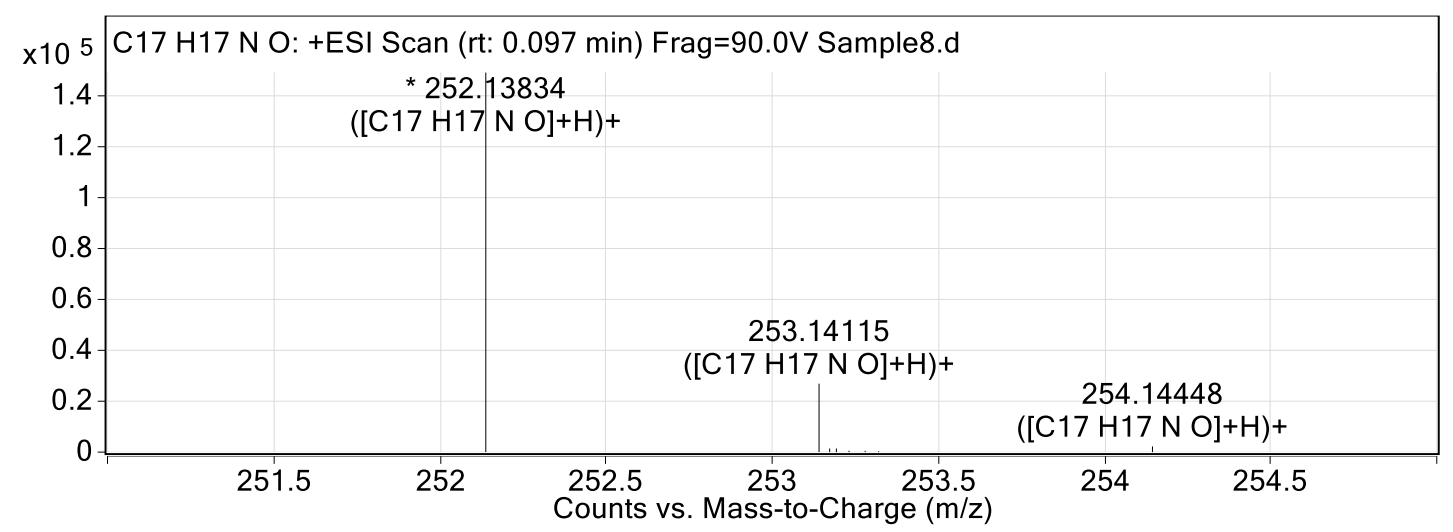

HRMS spectrum of $\mathbf{8 k}$

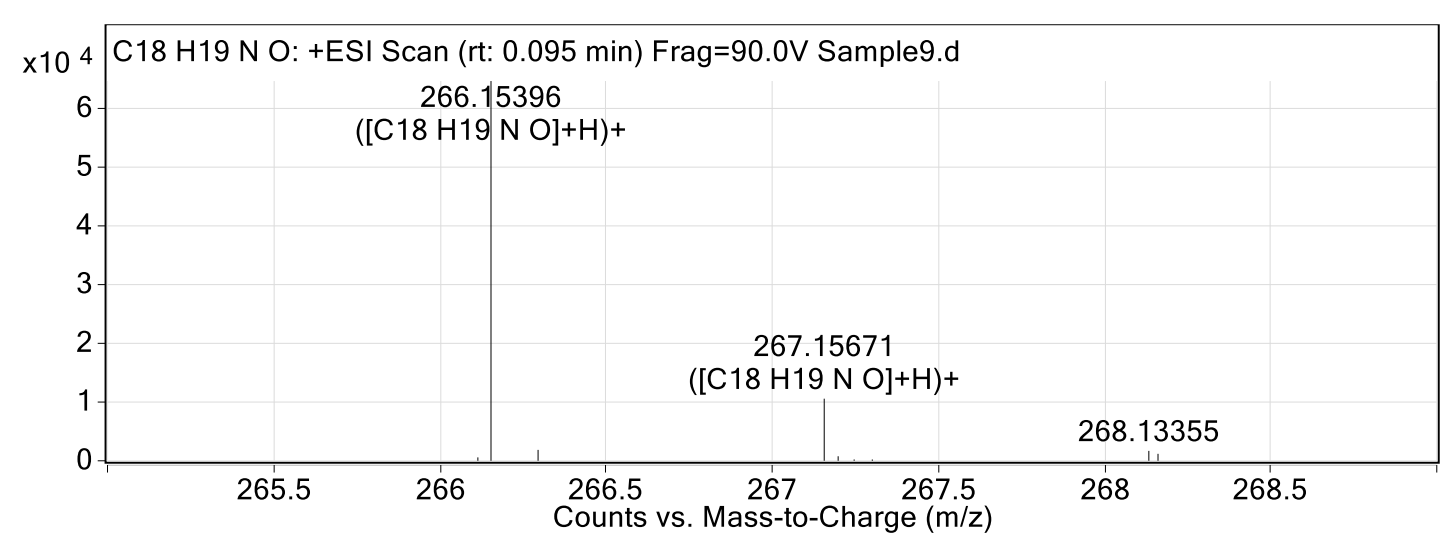

\section{HRMS spectrum of $\mathbf{8 1}$}

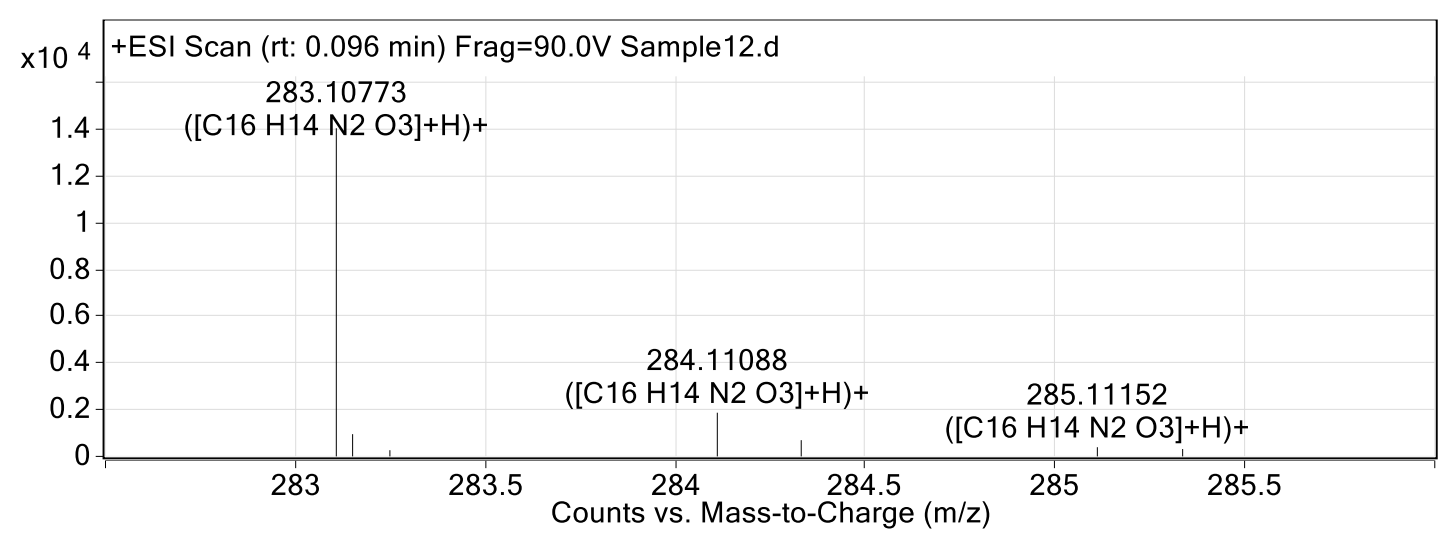


HRMS spectrum of $\mathbf{8 m}$

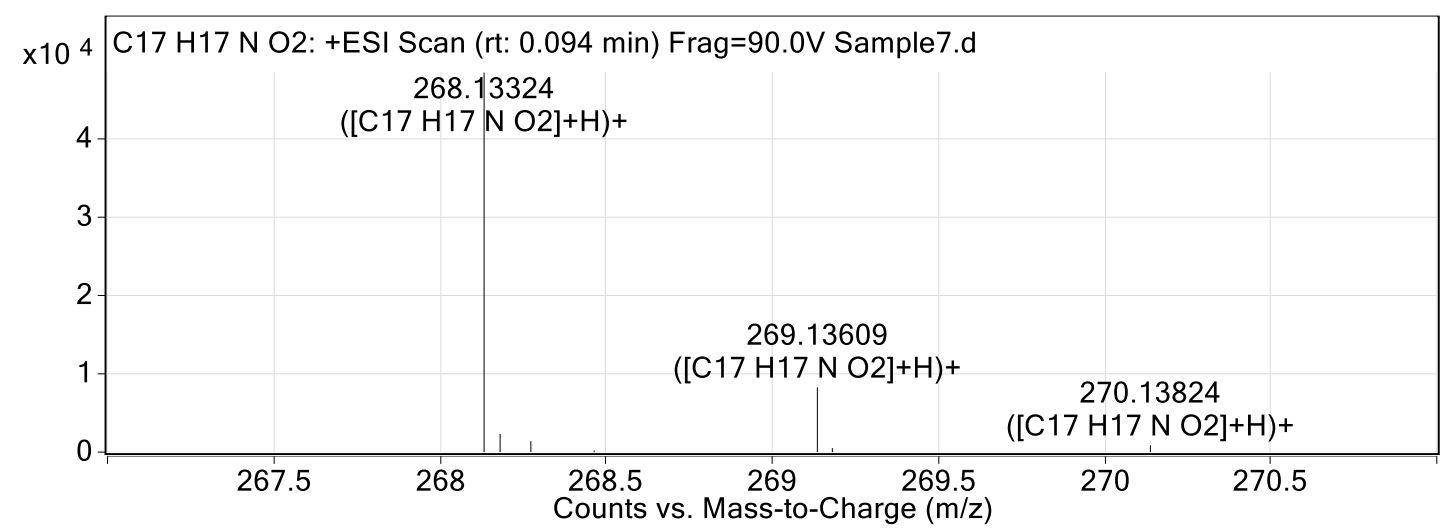

HRMS spectrum of $\mathbf{8 n}$

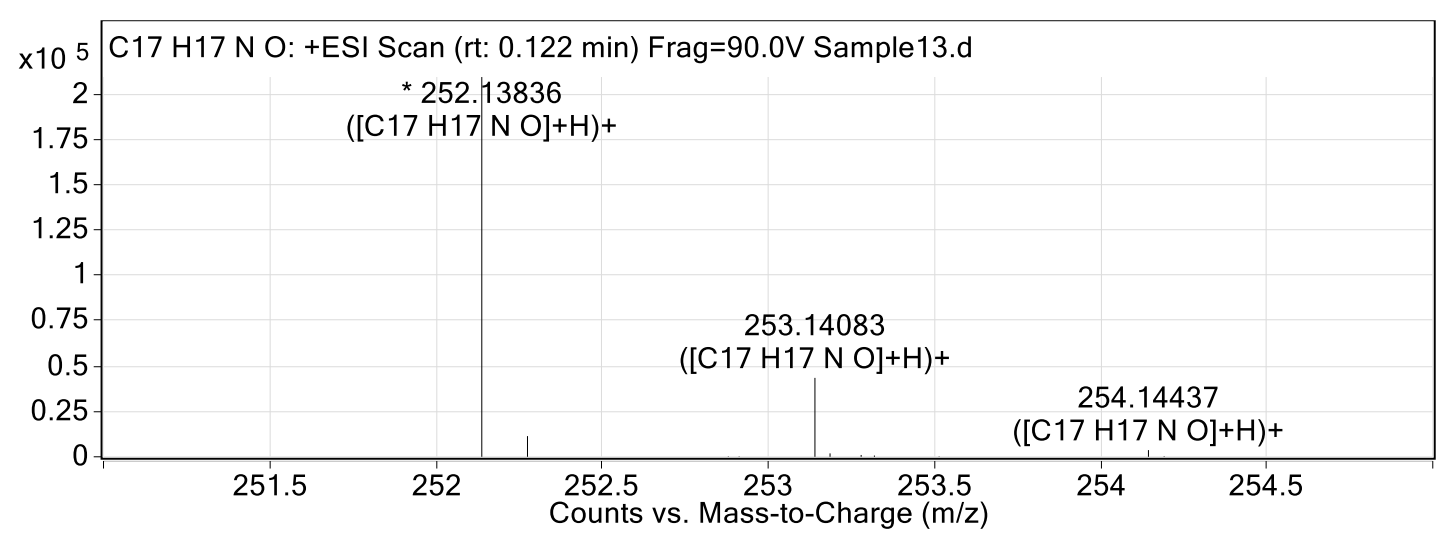

\section{HRMS spectrum of 80}

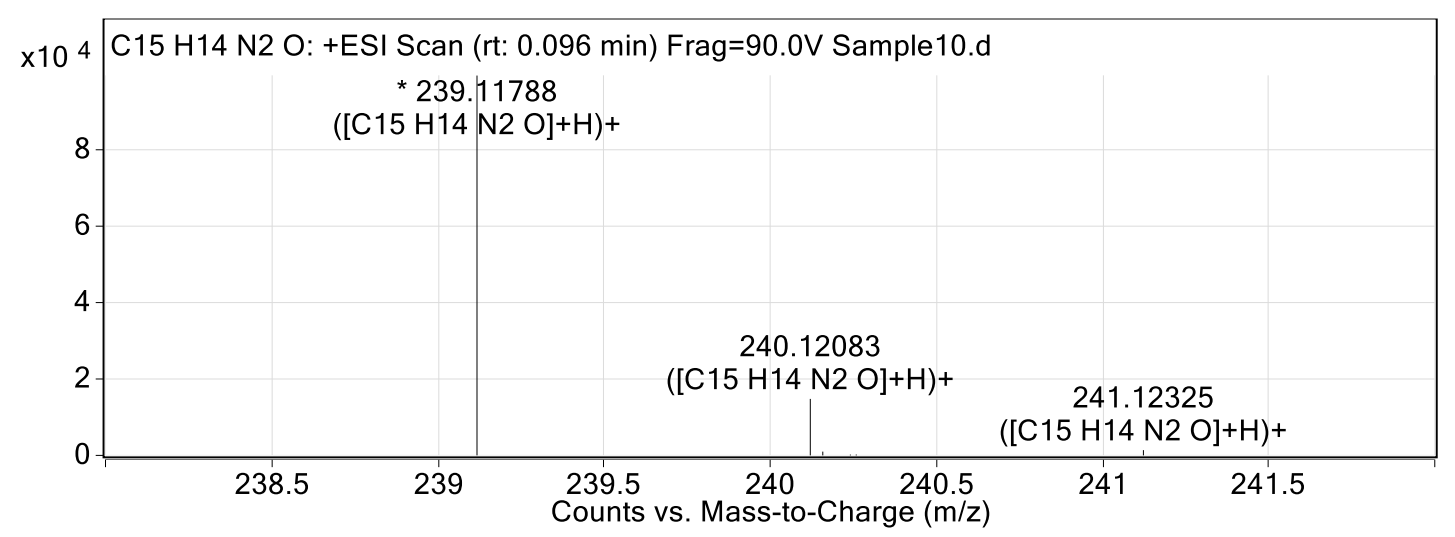


HRMS spectrum of $\mathbf{8 p}$

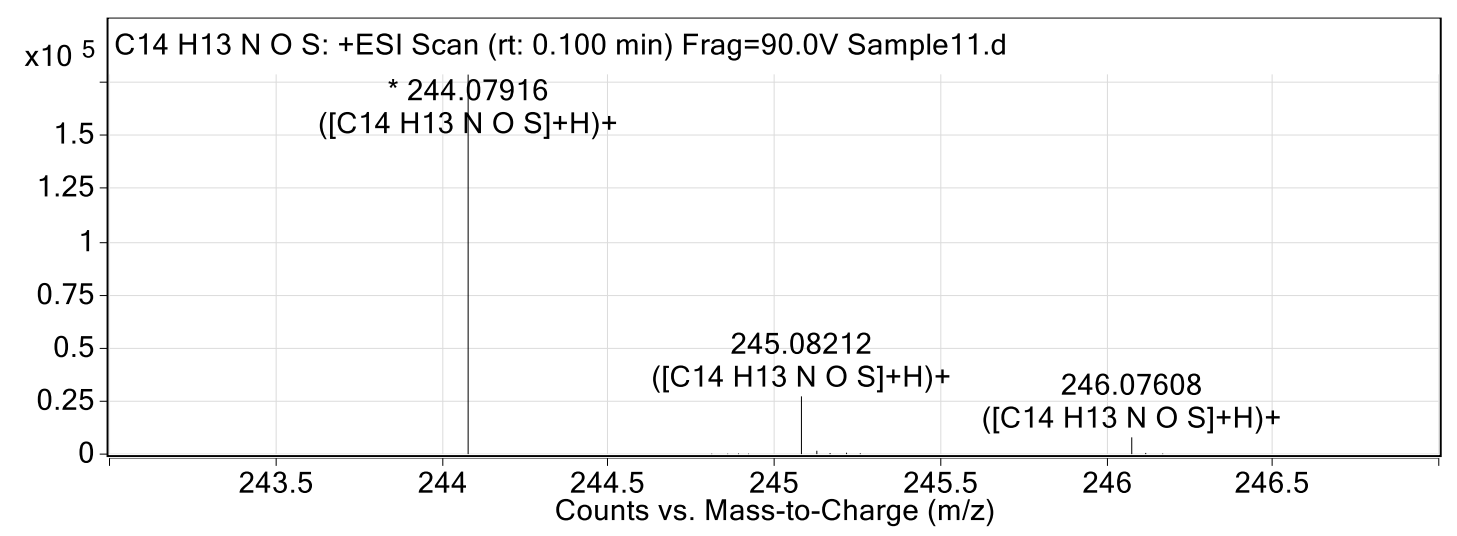

HRMS spectrum of $\mathbf{9 a}$

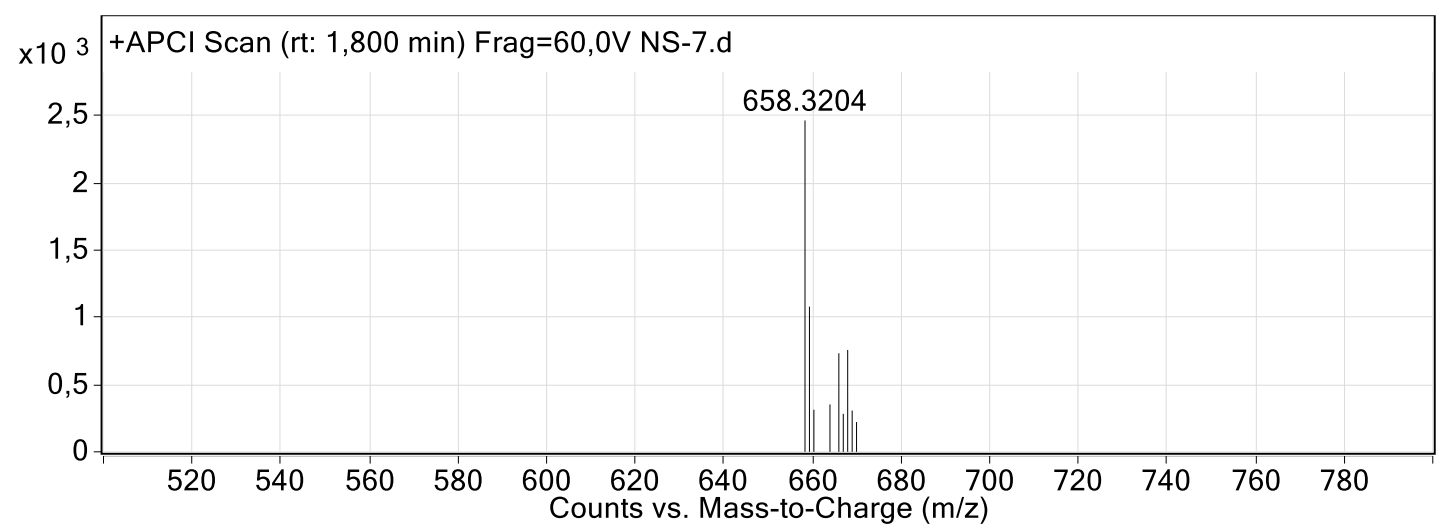

\section{HRMS spectrum of $\mathbf{9 b}$}

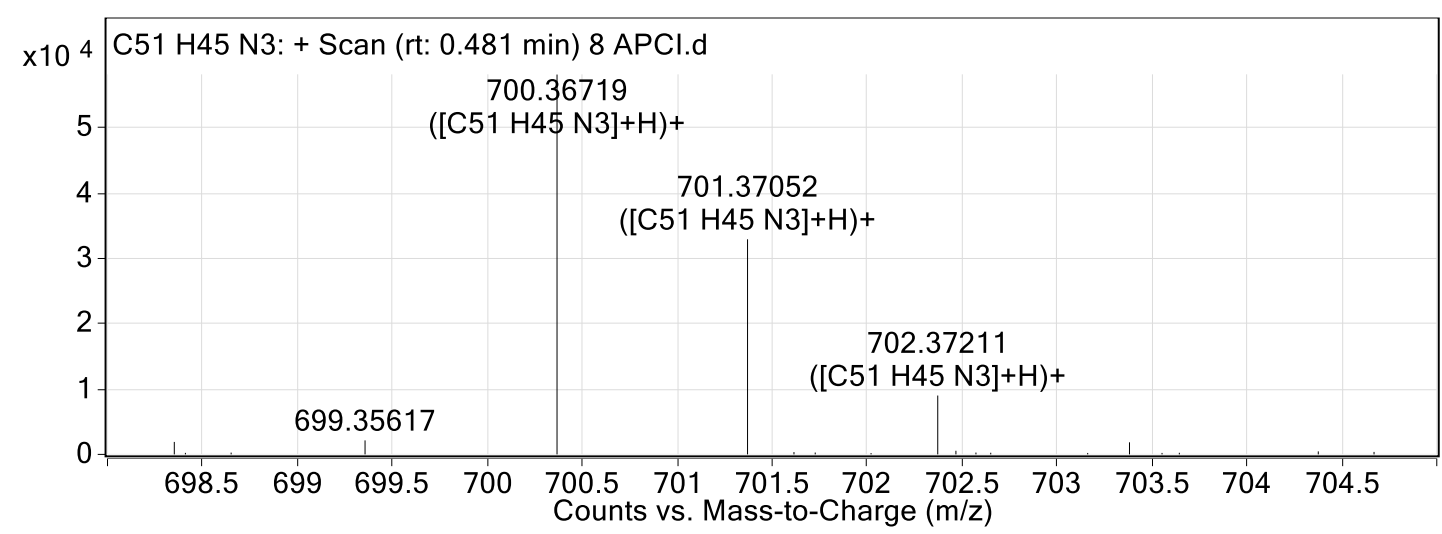


HRMS spectrum of $\mathbf{9 c}$

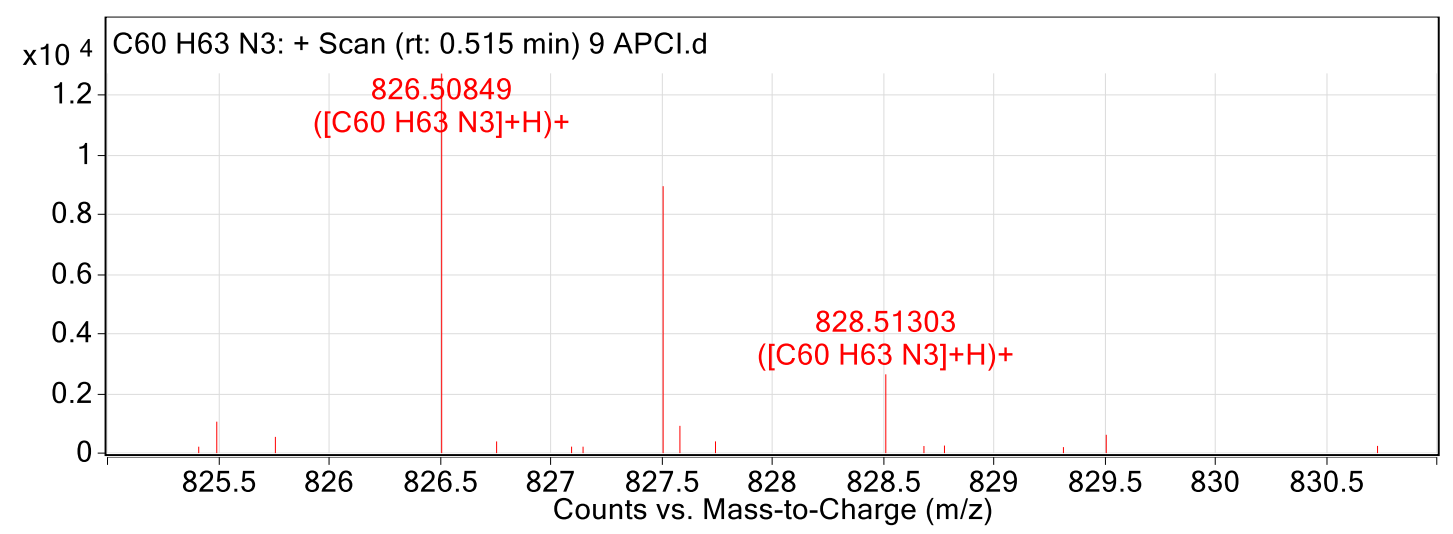

HRMS spectrum of 9d

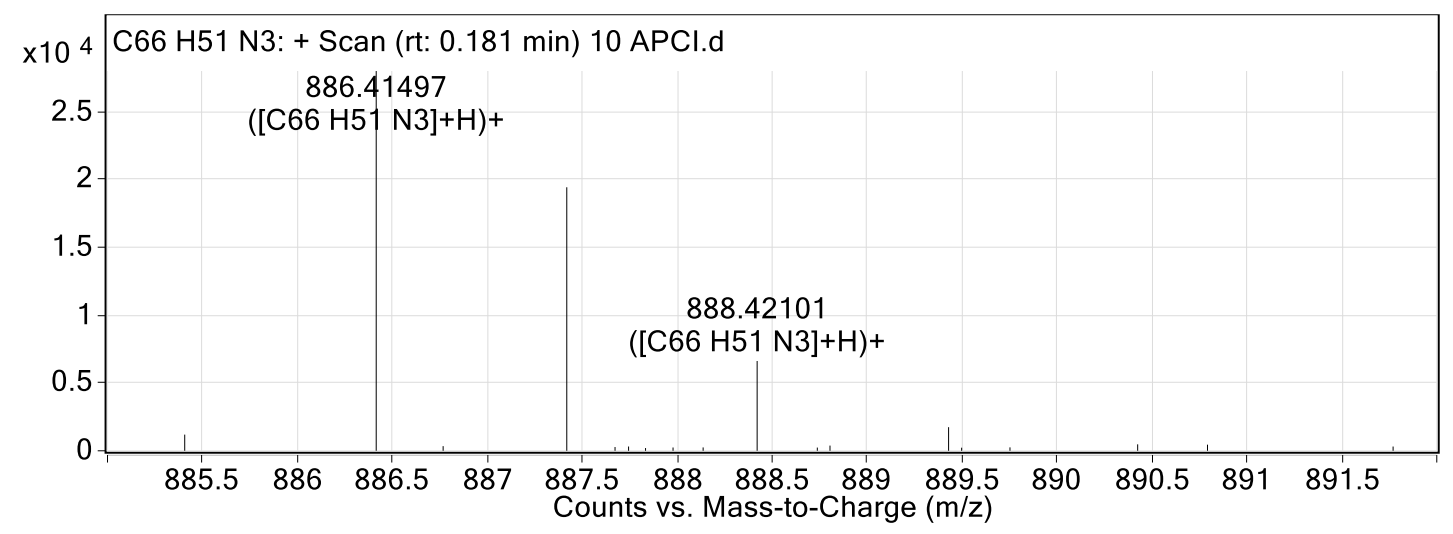

\section{HRMS spectrum of 11e}

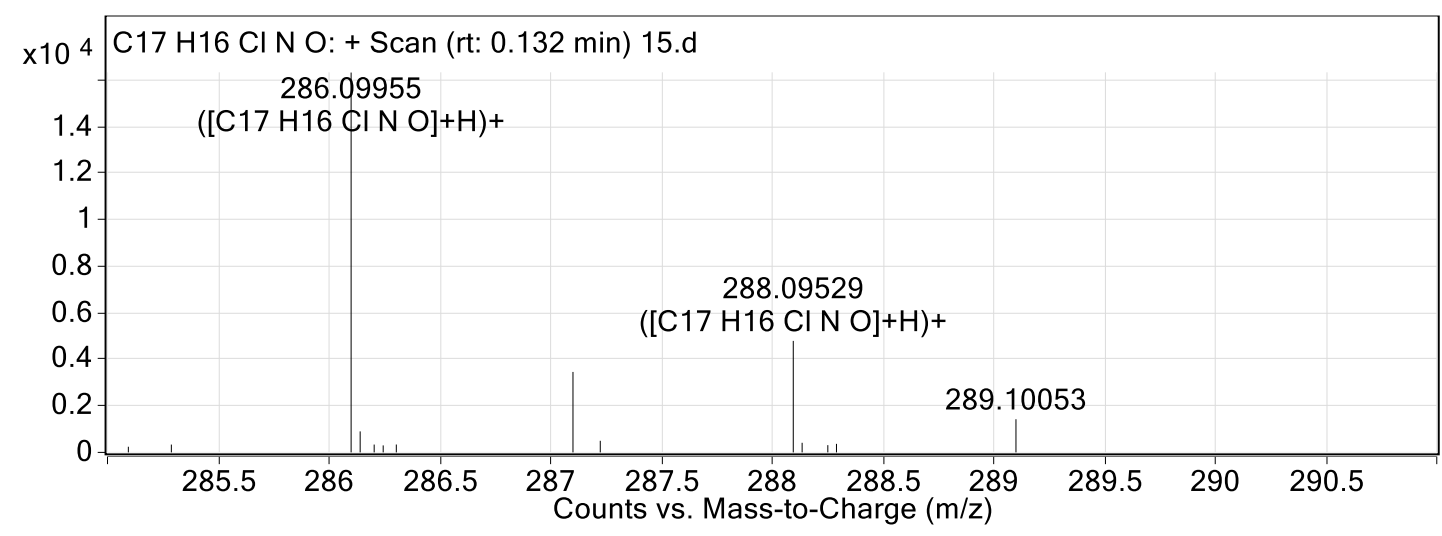


HRMS spectrum of $\mathbf{9 e}$

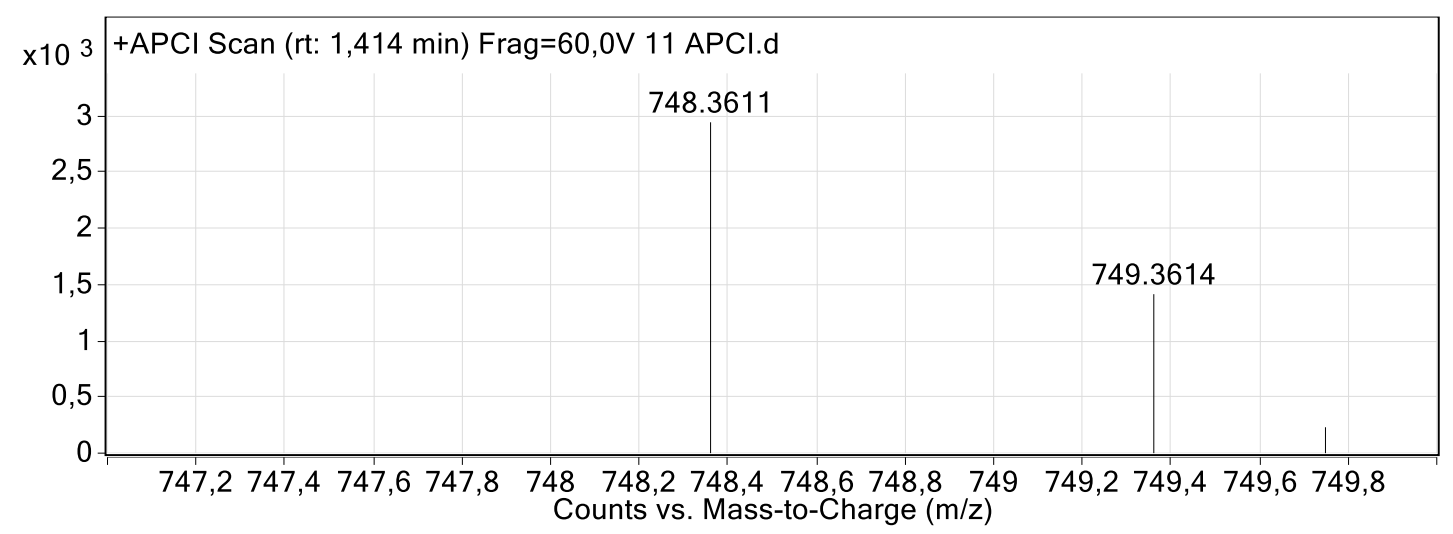

\section{HRMS spectrum of $\mathbf{1 1 f}$}

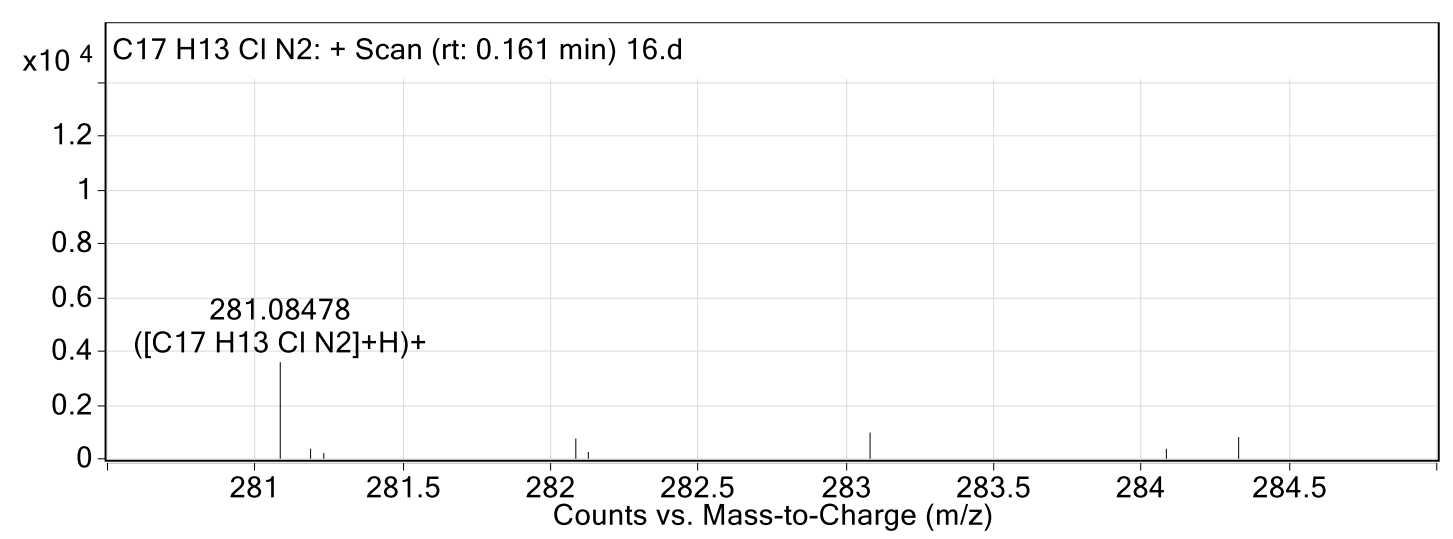

\section{HRMS spectrum of $\mathbf{1 1 g}$}

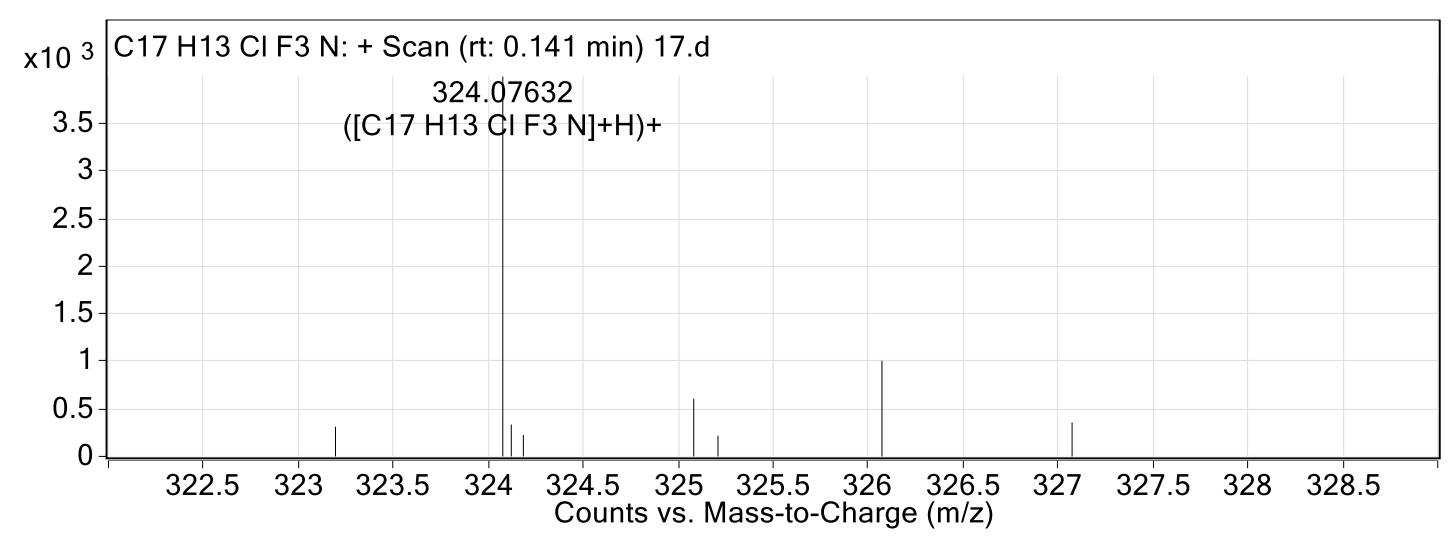


HRMS spectrum of $\mathbf{1 1 h}$

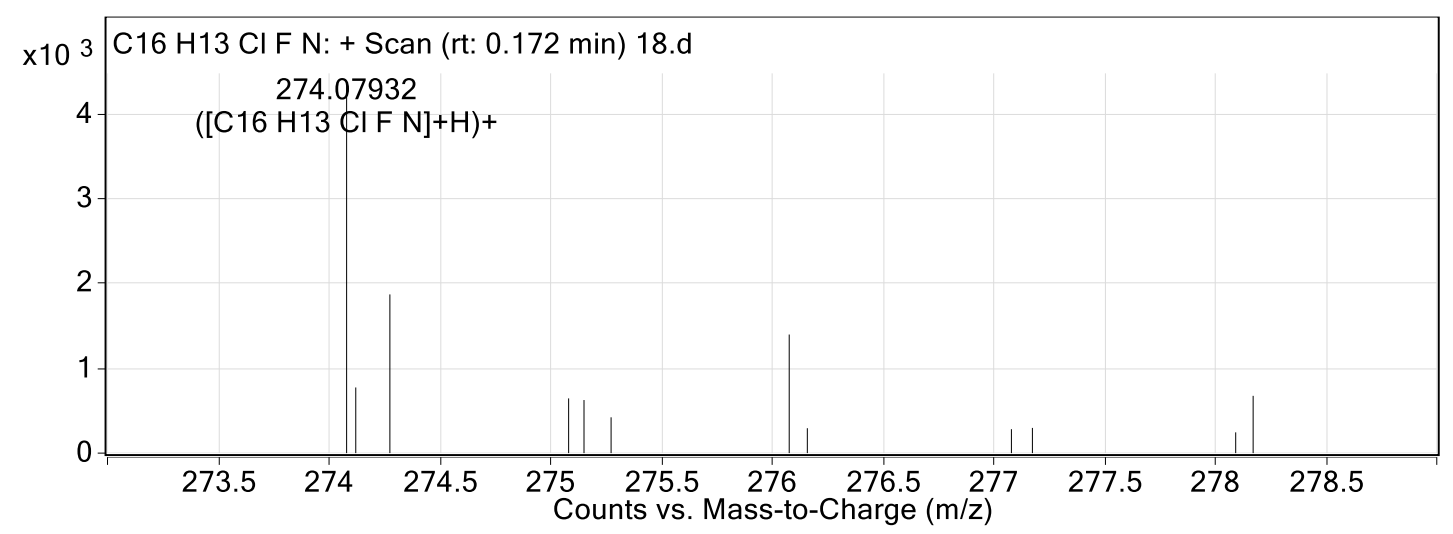

HRMS spectrum of $\mathbf{9 j}-\mathbf{A}$ and $\mathbf{9 j - B}$

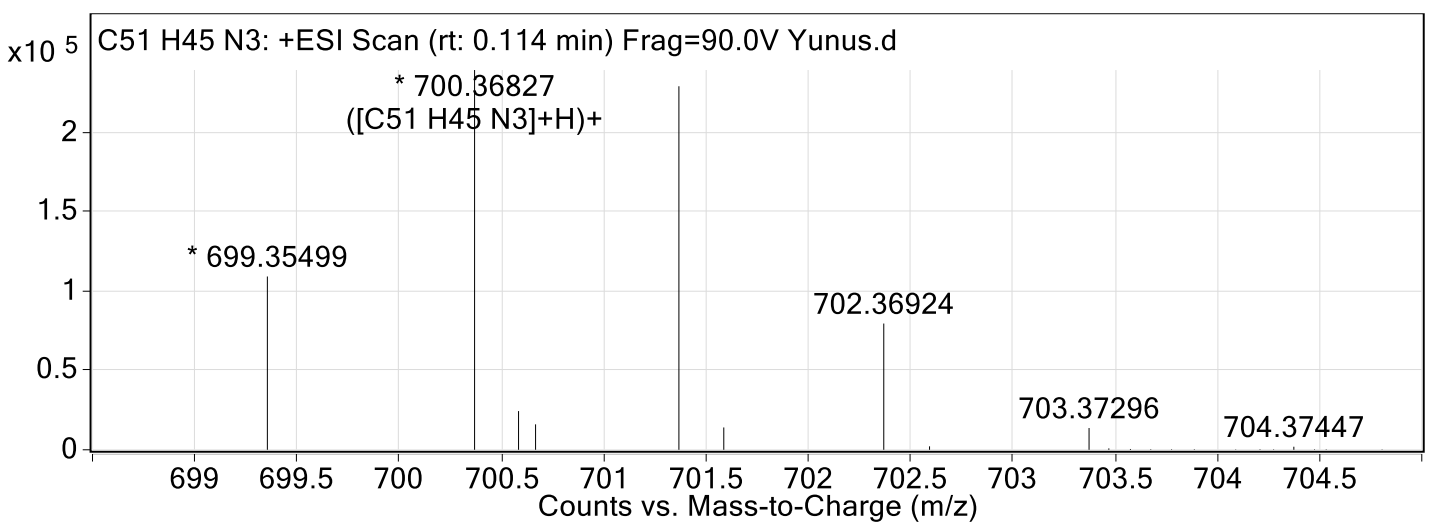

\section{HRMS spectrum of $\mathbf{9 k}$}

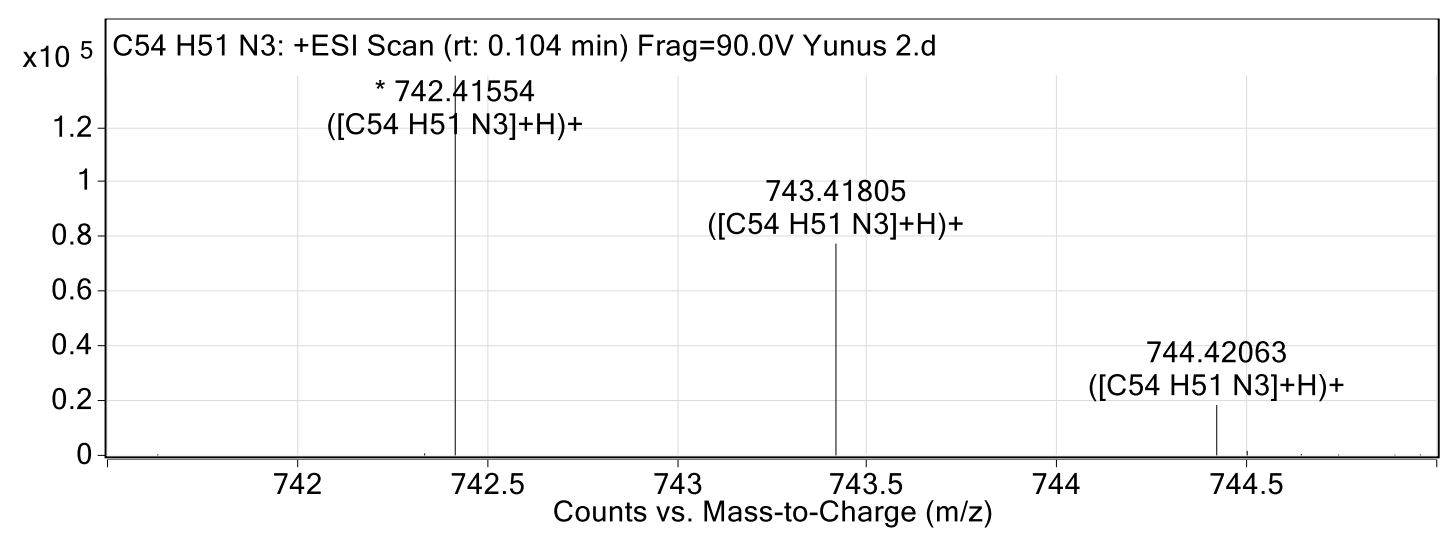


HRMS spectrum of $\mathbf{1 1 l}$

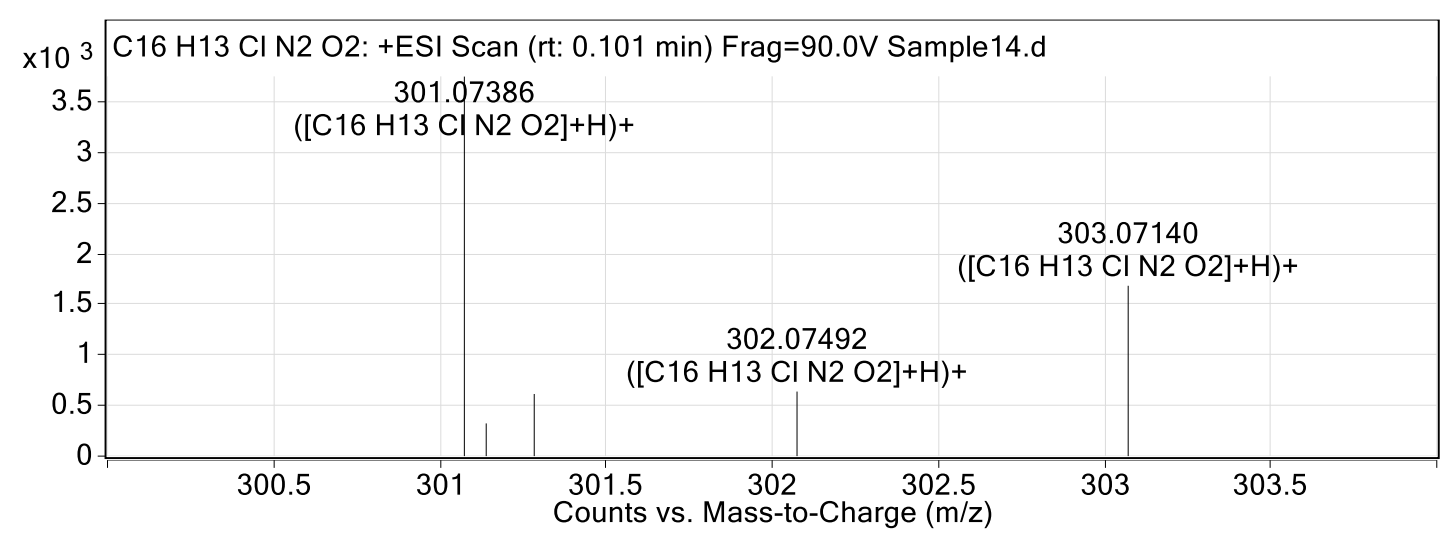

\section{HRMS spectrum of $9 \mathbf{m}$}

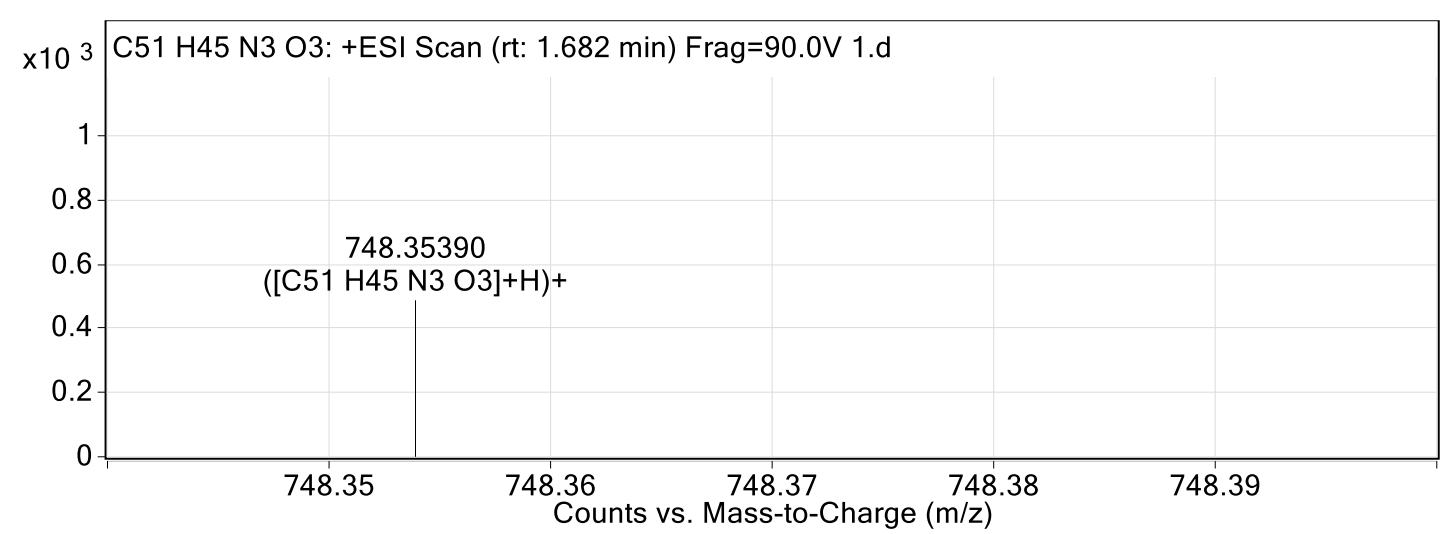

\section{HRMS spectrum of 9n}

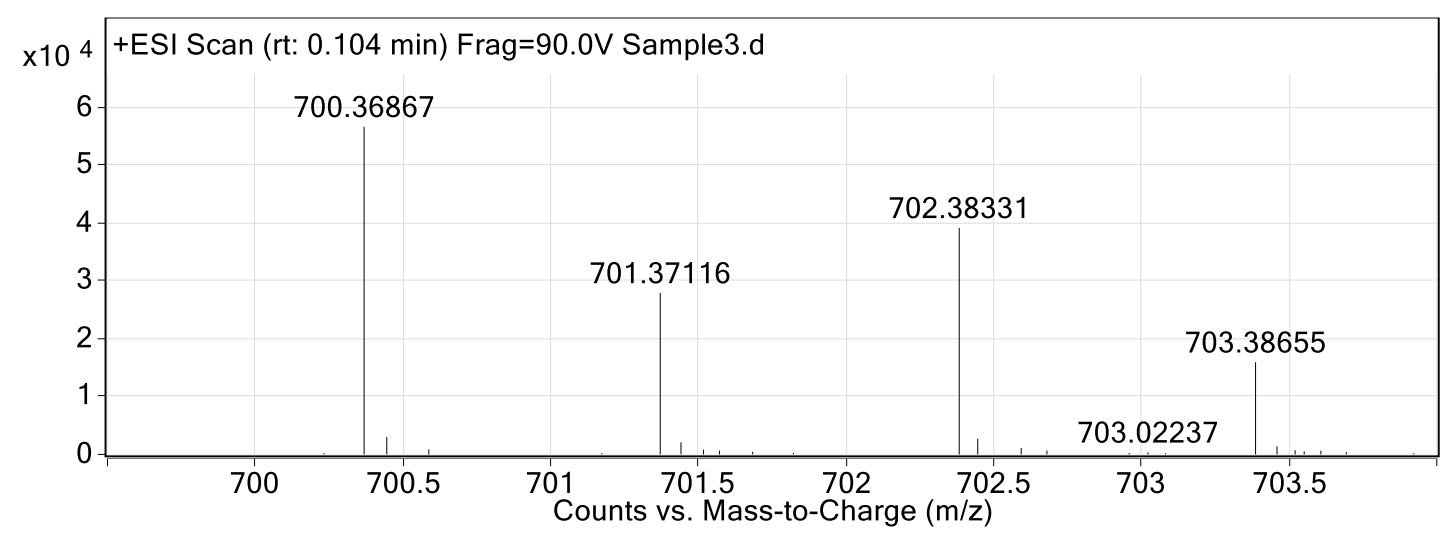




\section{HRMS spectrum of $\mathbf{1 1 0}$}

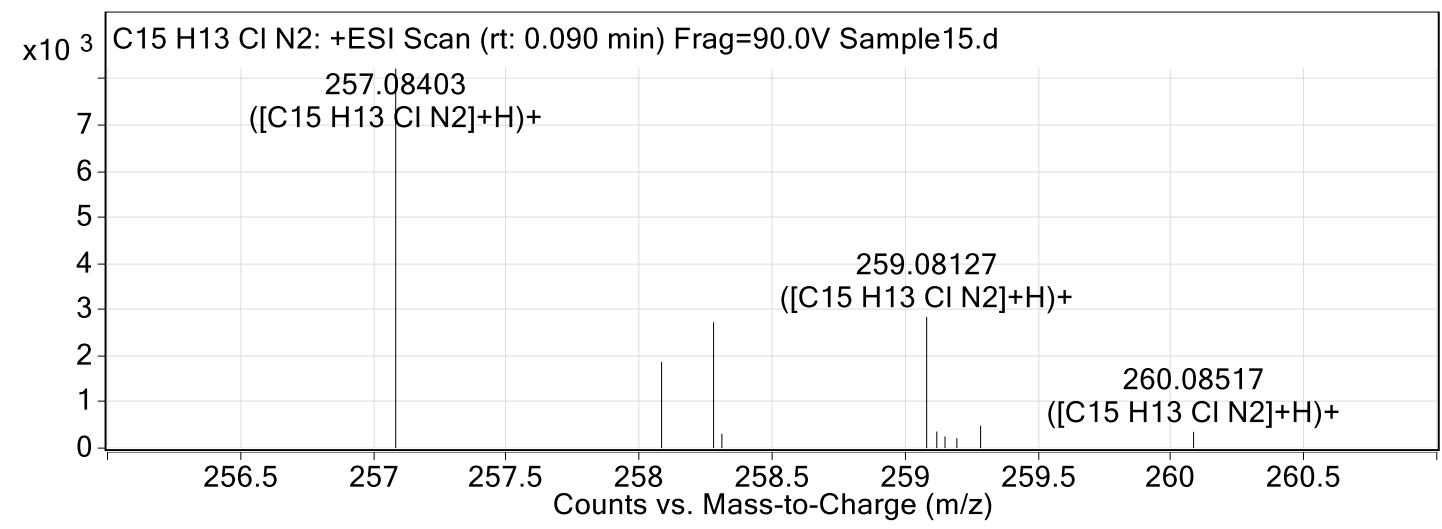

\section{HRMS spectrum of $\mathbf{9 p}$}

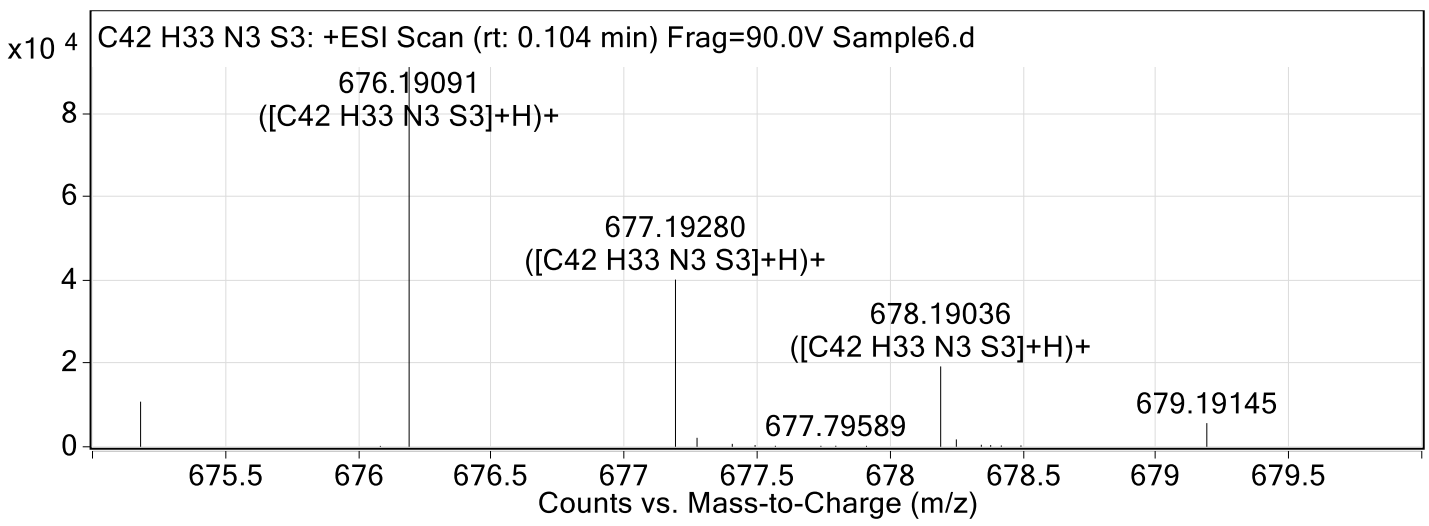

\section{HRMS spectrum of $\mathbf{1 0}$}

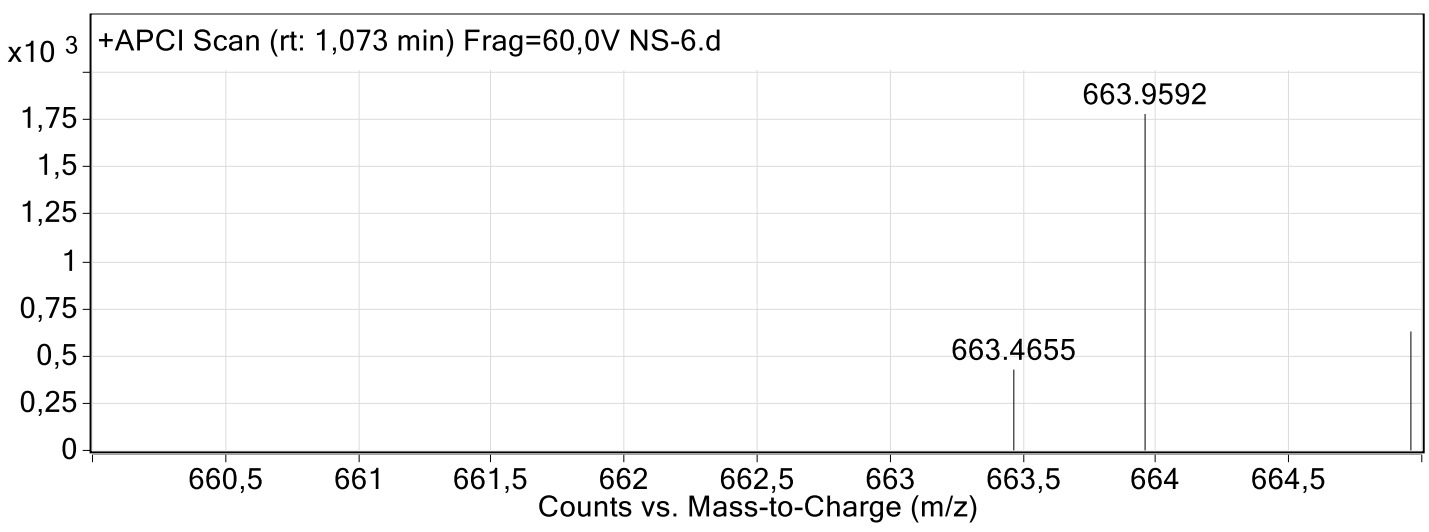


HRMS spectrum of $9 f$

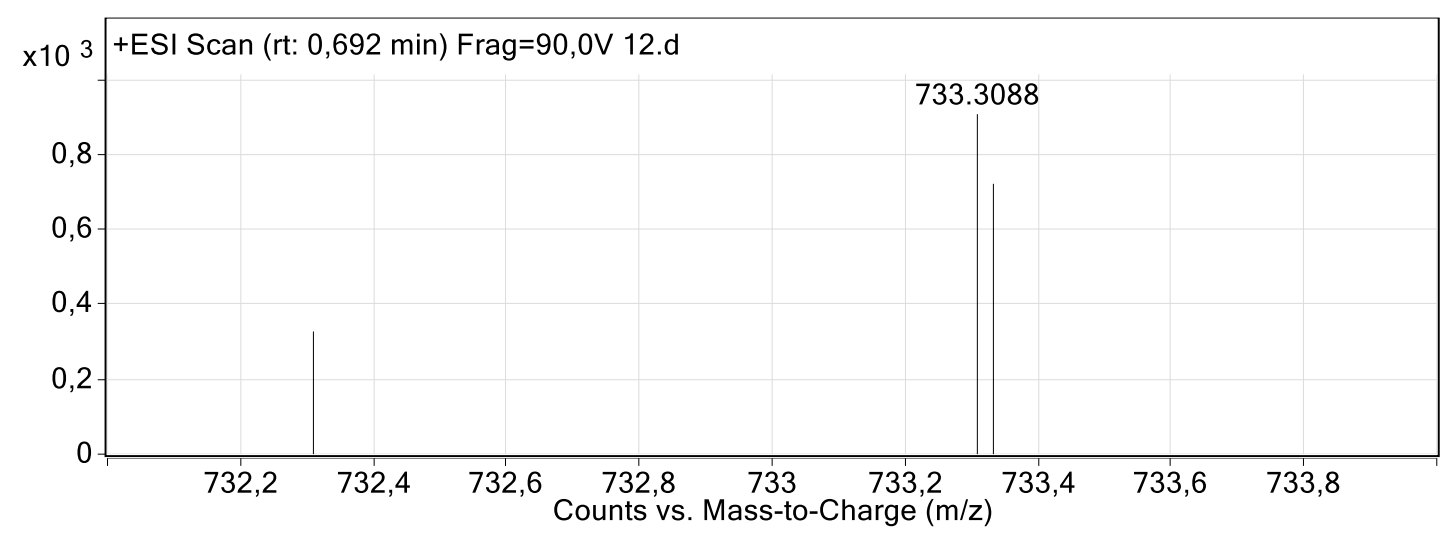

HRMS spectrum of $\mathbf{9 g}$

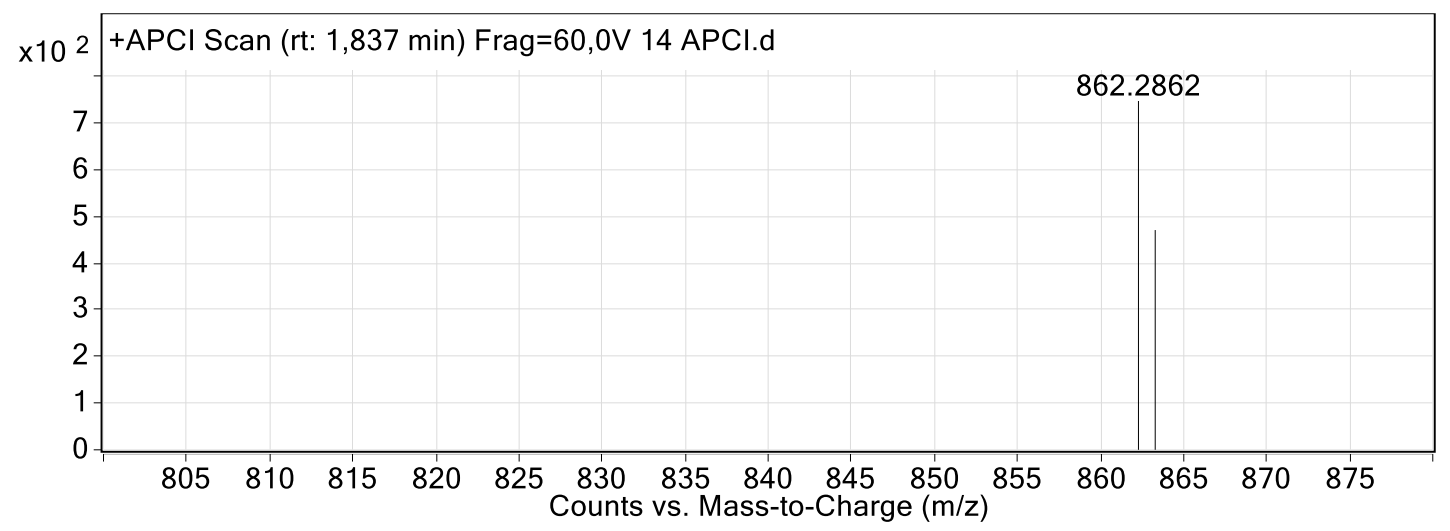

\section{HRMS spectrum of $\mathbf{9 h}$}

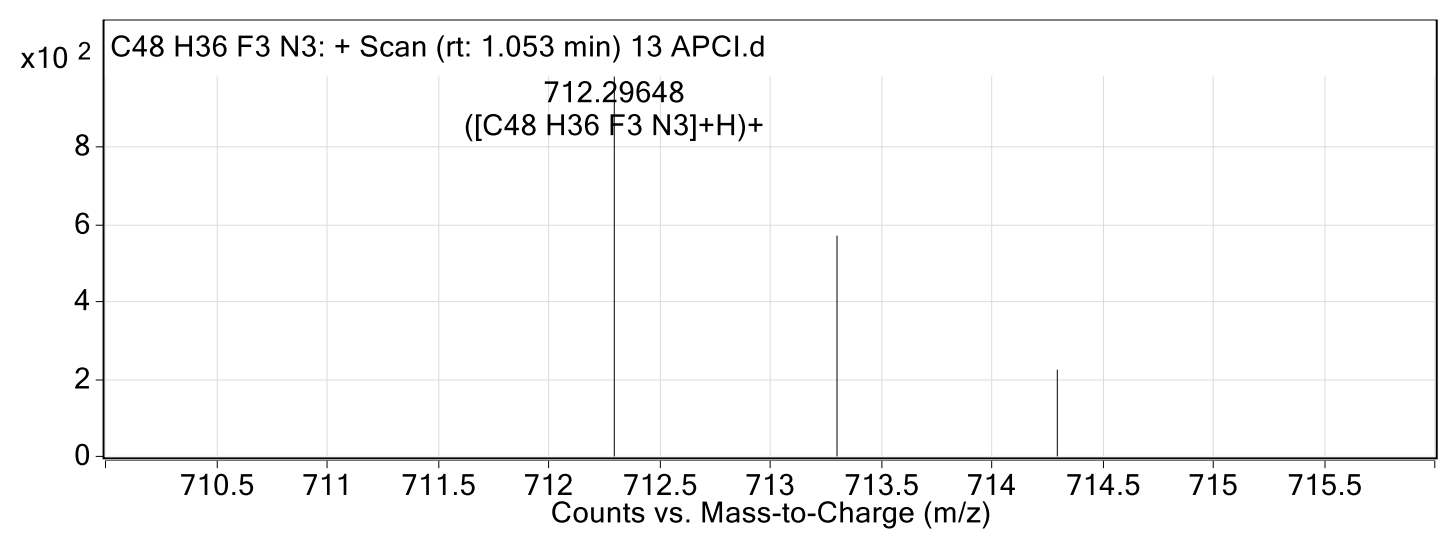




\section{HRMS spectrum of 91}

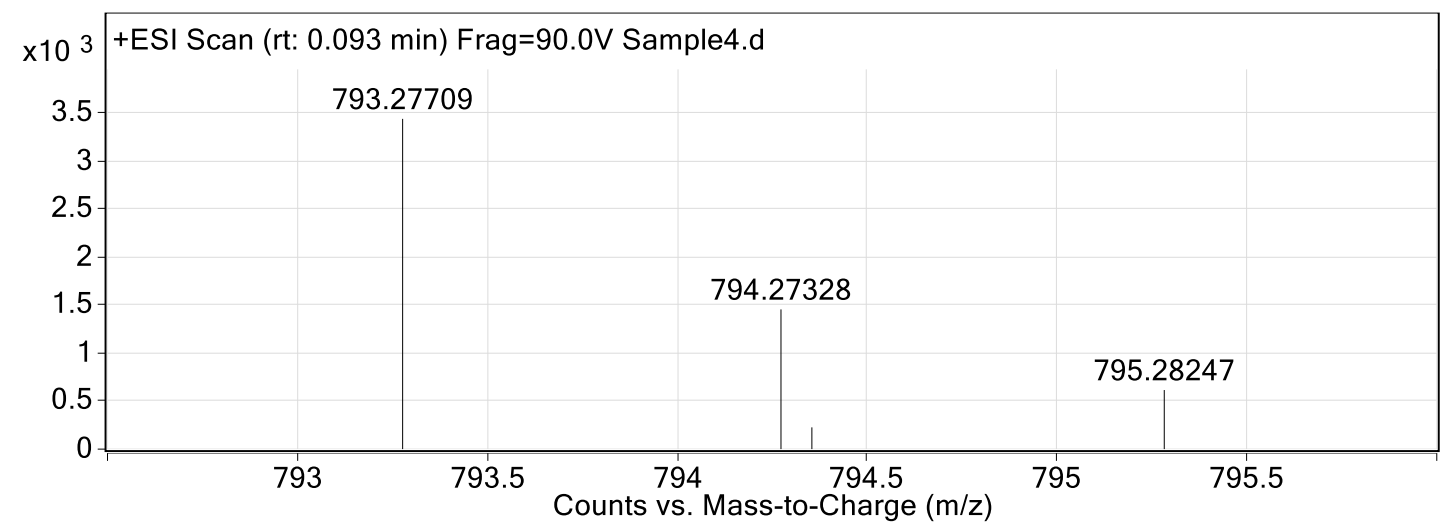

\section{HRMS spectrum of 90}

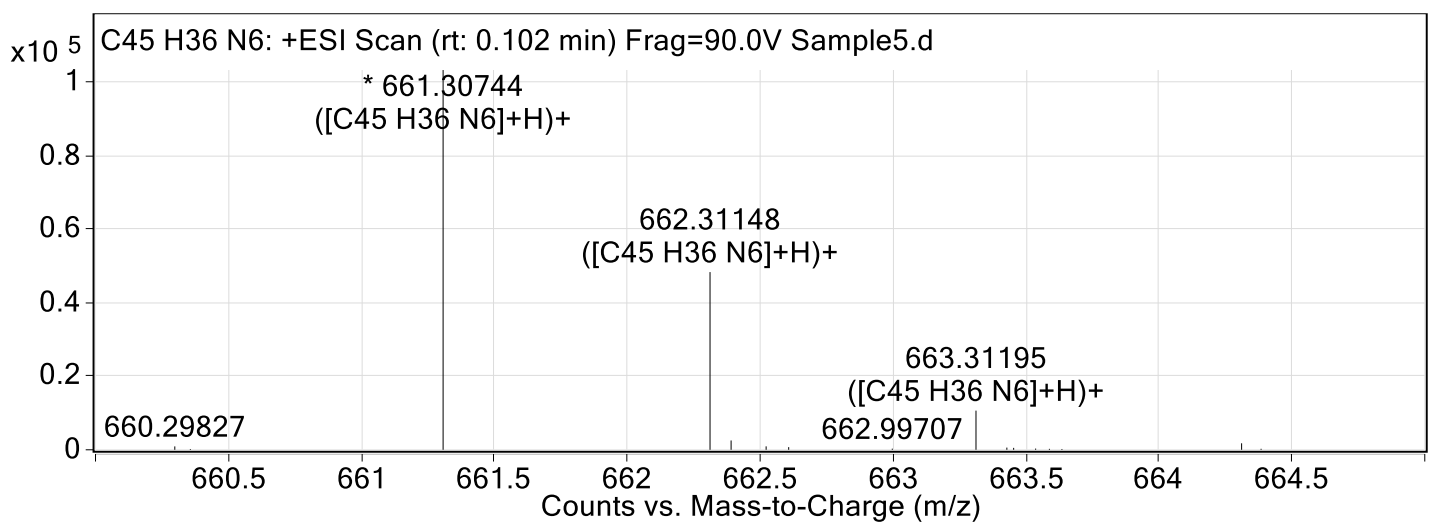

